\title{
Economic evaluation of three preventive drug therapies for osteoporotic fractures among women at different risk levels
}

Xin Gao

West Virginia University

Follow this and additional works at: https://researchrepository.wvu.edu/etd

\section{Recommended Citation}

Gao, Xin, "Economic evaluation of three preventive drug therapies for osteoporotic fractures among women at different risk levels" (2001). Graduate Theses, Dissertations, and Problem Reports. 1386.

https://researchrepository.wvu.edu/etd/1386

This Dissertation is protected by copyright and/or related rights. It has been brought to you by the The Research Repository @ WVU with permission from the rights-holder(s). You are free to use this Dissertation in any way that is permitted by the copyright and related rights legislation that applies to your use. For other uses you must obtain permission from the rights-holder(s) directly, unless additional rights are indicated by a Creative Commons license in the record and/ or on the work itself. This Dissertation has been accepted for inclusion in WVU Graduate Theses, Dissertations, and Problem Reports collection by an authorized administrator of The Research Repository @ WVU.

For more information, please contact researchrepository@mail.wvu.edu. 


\title{
Economic Evaluation of Three Preventive Drug Therapies for Osteoporotic Fractures among Women at Different Risk levels
}

\author{
Xin Gao \\ Dissertation submitted to the \\ School of Pharmacy \\ at West Virginia University \\ in partial fulfillment of the requirements \\ for the degree of \\ Doctor of Philosophy \\ in \\ Pharmaceutical Systems \& Policy \\ Suresh Madhavan, Ph.D, MBA, Chair \\ David Nau, Ph.D. \\ Syed Islam, M.D., Ph.D. \\ Mayur Amonkar, Ph.D. \\ Sidney Rosenbluth, Ph.D. \\ Ambarish Ambegaonkar, Ph.D. \\ Department of Pharmaceutical Systems \& Policy
}

Morgantown, West Virginia

2001

Keywords: Economic Evaluation, Prevention, Osteoporotic Fracture, Women

Copyright 2001 Xin Gao 


\title{
ABSTRACT \\ Economic Evaluation of Three Preventive Drug Therapies for Osteoporotic Fractures among Women at Different Risk levels
}

\begin{abstract}
Xin Gao
Objective: To evaluate cost-effectiveness (CE) of three drug therapies for preventing osteoporotic fractures in postmenopausal women from a state Medicaid Program perspective using the estimated risk distribution in the study population. The three therapies are: hormone replacement therapy (HRT), alendronate, and raloxifene. Methods: A hypothetical cohort of white women aged 45-85 years, postmenopausal, and without past incidence of osteoporotic fractures was treated with one of the three alternatives, and tracked over 3 years in a decision model. The CE ratio was defined as the treatment costs [e.g., medications, monitoring, adverse events (AE)] divided by the number of fractures averted. Treatment Willingness-To-Continue (WTC) rate was also considered. Data were collected from literature, expert panel survey, Medicaid claims data, and a risk survey in the study population. Monte Carlo simulations were conducted (distributions used: background risk, cost, and risk reduction rate). Risk or probability of osteoporotic fracture was also divided into three strata: low (risk $<0.1)$, medium $(0.1 \leq$ risk $<0.3)$, and high (risk $\geq 0.3$ ). Results: Compared to no therapy, the expected CE of HRT was $\$ 29,119$ per fracture averted, alendronate: $\$ 35,101$, and raloxifene: $\$ 39,760$. The incremental CE was $\$ 42,181$ for alendronate (relative to HRT) and $\$ 85,509$ for raloxifene (relative to alendronate). The incremental CE of alendronate and raloxifene were \$151,981 and \$697,270 among women with low risk (43\% of the sample), compared to $\$ 11,099$ and $\$ 34,017$ respectively among high-risk women ( $26 \%$ of the sample). CE was not sensitive to discount rate and AE probabilities. Conclusions: HRT is the most cost-effective strategy even though it may have relatively high monitoring and AE costs, and low WTC rate. The significant decrease in marginal costs of Alendronate and Raloxifene in high-risk women indicates an economic condition to use these two drugs. The study provides a framework to make risk-appropriate coverage decisions for chemopreventive agents.
\end{abstract}




\title{
Dedicated to
}

\author{
My Parents \\ Zhenchuan Gao and Ying Sun \\ My Husband \\ Shen Xiao
}




\section{ACKNOWLEDGEMENTS}

There are many people who have made this study possible. I would first like to express my gratitude to Dr. Suresh Madhavan, my advisor and Committee Chairperson, for his guidance, knowledge, and friendship that he offered throughout the course of this study. He is an inspiring teacher and I feel very fortunate to have trained underneath him. He has given me important skills I need for success. For this I am deeply indebted.

I would also like to thank my committee members Dr. David Nau, Dr. Syed Islam, Dr. Mayur Amonkar, Dr. Sidney Rosenbluth, and Dr. Ambarish Ambegaonkar for their invaluable suggestions and support throughout this study. A very large thank-you goes out to every one who contributed with my study. These people are Dr. Irma Ullrich, Dr. Roxann Powers, Dr. Barbara Kaplan-Machlis, and Dr. Virginia Scott. I would also like to extend my thanks to the staff of School of Pharmacy and all the graduate students that gave me their kindly support, encouragement, and companion. Additional thanks go to Pfizer Inc. for financially supporting my research.

Finally, those who deserve the greatest credit for everything that I have accomplished, not just in graduate school but also in life, are my parents and my husband. They give me the lifetime of love, understanding, encouragement, and everything. They are always there taking care of me and giving me the strongest support. Words cannot express my appreciation. I dedicate this dissertation to them and want them to know they are the best and I love them so much. 


\section{TABLE OF CONTENTS}

\section{PAGE}

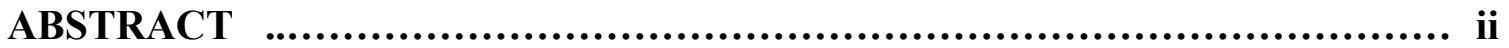

DEDICATION

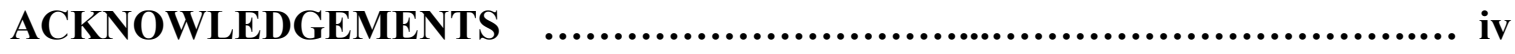

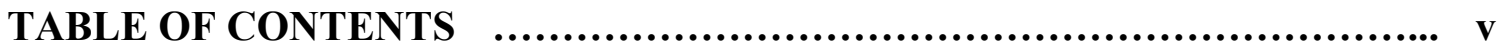

LIST OF TABLES $\quad$ T............................................................. ix

LIST OF FIGURES $\quad$ (.................................................................. xi

\section{CHAPTER I . INTRODUCTION}

1. Epidemiology and Costs of Osteoporosis and Osteoporotic Fracture .. 1

2. Risk Factors Associated with Osteoporosis and Fracture $\ldots . \ldots \ldots \ldots \ldots . .5$

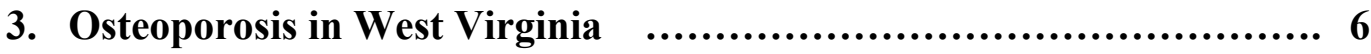

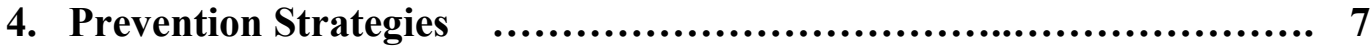

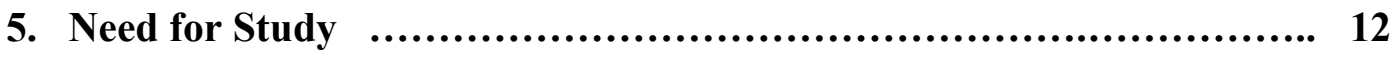

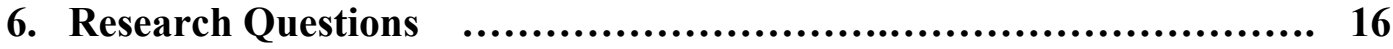

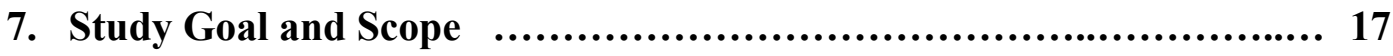

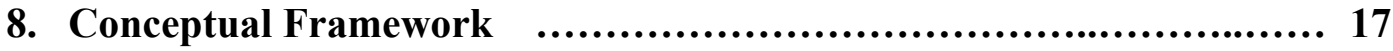

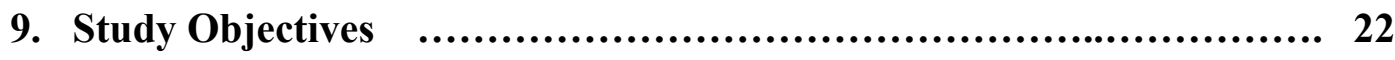

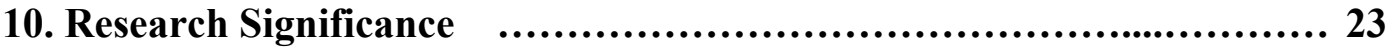

CHAPTER II . LITERATURE REVIEW 
1. Postmenopausal Osteoporosis and Related Fractures ................ 26

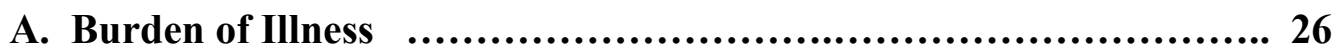

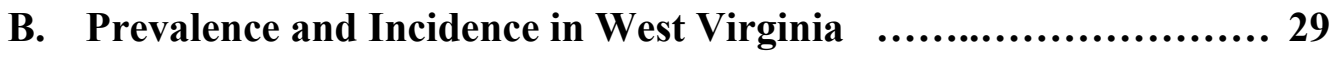

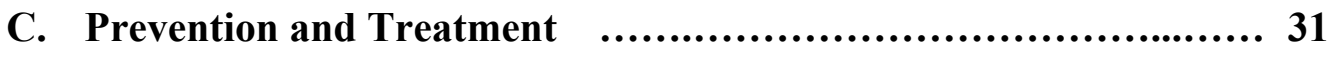

2. Pharmacotherapy in the Treatment and Prevention of Osteoporosis ... 32

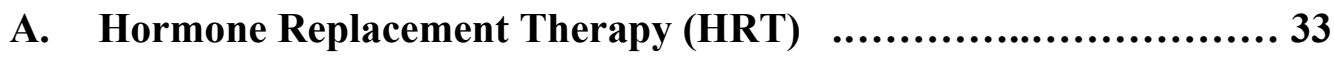

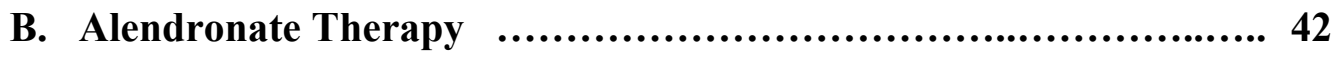

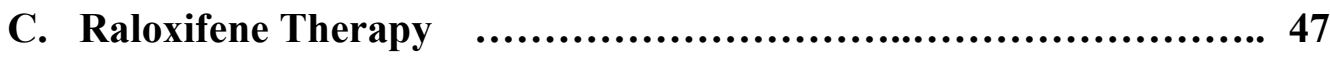

3. Pharmacoeconomic Research On The Three Drug Therapies ........... 52

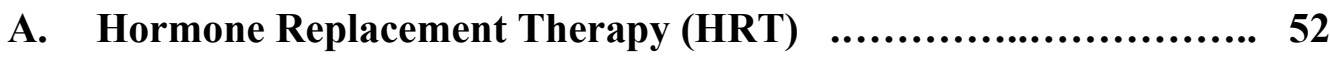

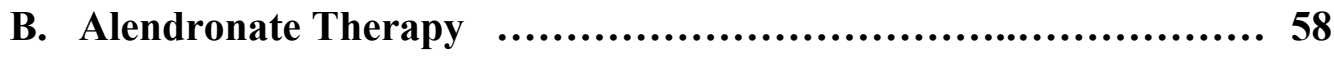

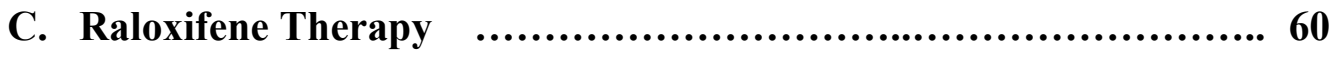

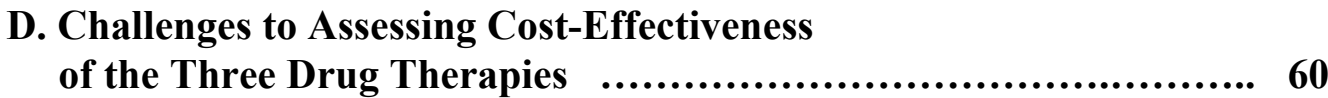

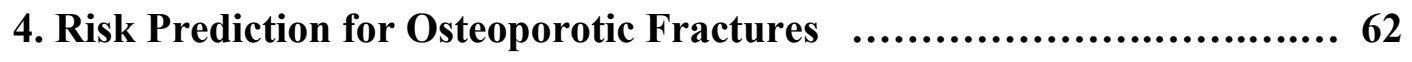

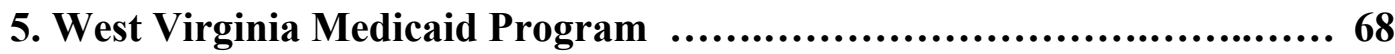

\section{CHAPTER III. METHODS}

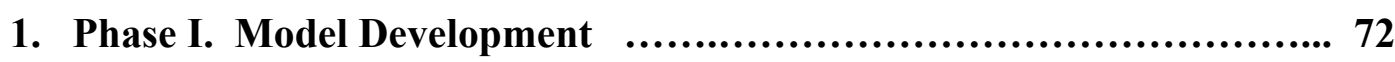

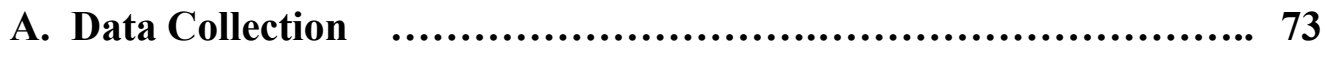

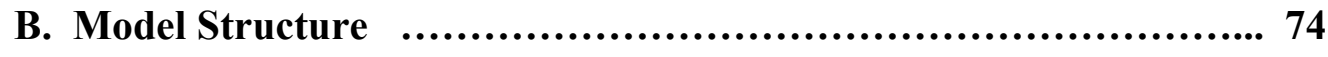

C. Main Assumptions $\ldots \ldots \ldots \ldots \ldots \ldots \ldots \ldots \ldots \ldots \ldots \ldots \ldots \ldots \ldots \ldots \ldots \ldots \ldots \ldots . . \ldots \ldots 1$

2. Phase II. Analysis of Background Risk ............................ 83

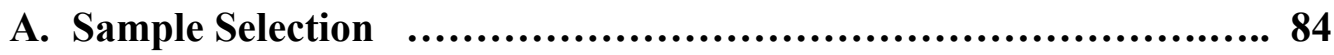


B. Sample Size Estimation …............................................. 87

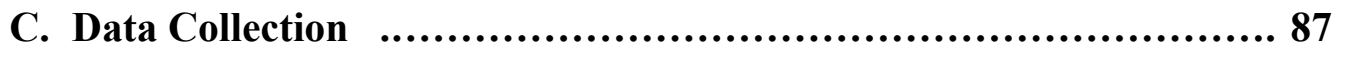

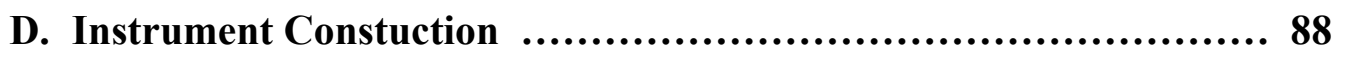

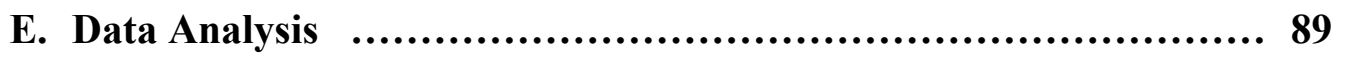

F. Database Management …......................................... 92

4. Phase III. Cost-Effectiveness Analysis ................................. 92

A. Conventional Cost-Effectiveness Analysis Using Mean Values ...... 92

B. Probabilistic Analysis and Monte Carlo Simulation $\ldots . . . \ldots \ldots \ldots . . . . . . .93$

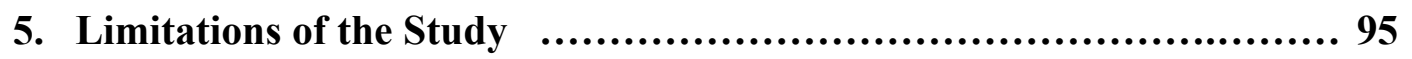

\section{CHAPTER IV. RESULTS}

1. Phase I. Model Development …......................................... 99

A. Decision Tree Structure and Relevant Variables ........................ 99

2. Phase II. Analysis of Background Risk ................................. 116

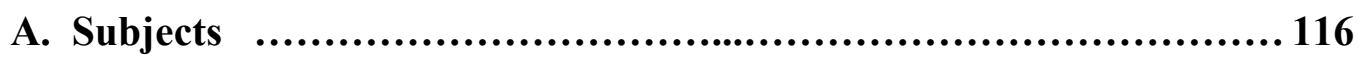

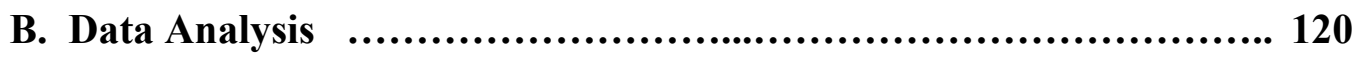

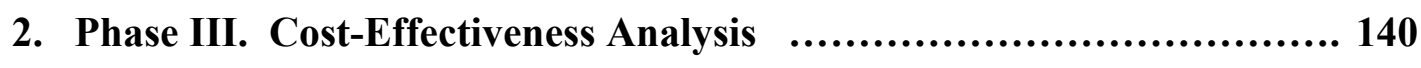

A. Cost-Effectiveness Analysis Using Mean Values ...................... 140

B. Probabilistic Analysis and Monte Carlo Simulation $\quad$................... 147 


\section{CHAPTER V. DISCUSSION AND RECOMMENDATIONS}

1. Study Review ....................................................... 152

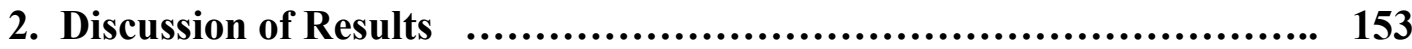

3. Implications of the Study Results $\quad$....................................... 165

4. Recommendations for Future Research .................................. 167

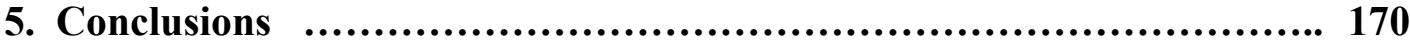

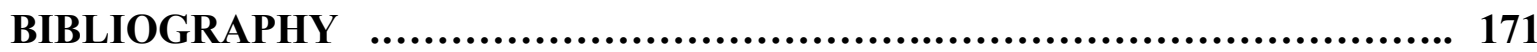

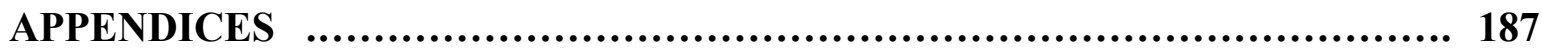

A. Average Wholesale Prices Used by WV Medicaid Program ............. 187

B. List of CPT Codes Used in the Study $\quad$..................................... 189

C. Expert Panel Survey .................................................... 191

D. List of ICD-9 Codes Used in the Study $\quad$................................. 195

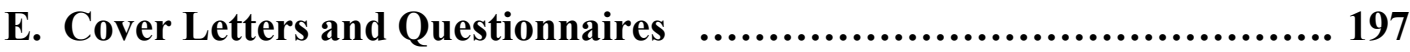

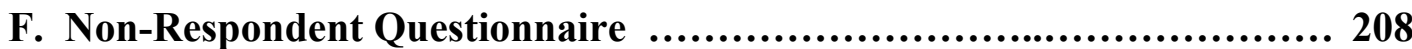

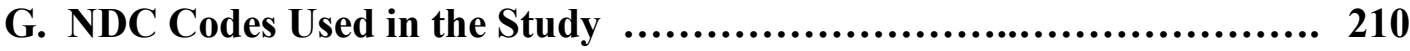




\section{LIST OF TABLES}

TABLE

PAGE

Table 4-1. Therapy Formulations and Patient Distribution for Each Dosage Form Based on Clinical Guidelines and Delphi Panel Estimates 102

Table 4-2. Medication Costs (1999 dollars) $\quad$........................................ 103

Table 4-3. Average Monitoring Costs During the 3-year Treatment Period ......... 105

Table 4-4. Adverse Events and Related Treatment Costs $\quad$............................. 107

Table 4-5. Complete Treatment Costs Summary …................................. 109

Table 4-6. Treatment Willingness-To-Continue (WTC) Rate (\%) .................. 111

Table 4-7. Treatment Costs Summary for Discontinuation Group ................. 112

Table 4-8. Risk Reduction Rates Used for the Three

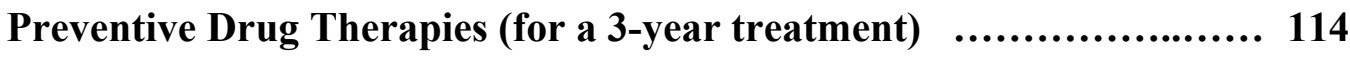

Table 4-9. The Value of Costs and Probability Variables ............................. 115

Table 4-10. General Comparisons of Cases and Controls on Categorical Variables ................................ 122

Table 4-11. Valid N and Value Range for Continuous Variables ................... 124

Table 4-12. General Comparisons of Cases

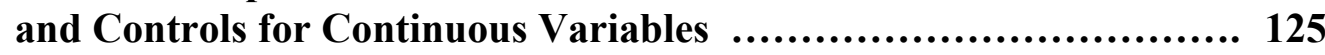

Table 4-13. Comorbidity and the Use of Drugs that May Affect Osteoporotic Fracture Incidence among Cases and Controls .......... 127

Table 4-14. The Relationship of Osteoporotic Fractures and Selected Risk Factors 129

Table 4-15. Prediction Power of the Result Model Based on Selected Cases $(\mathrm{N}=\mathbf{3 2 3})$

Table 4-16. Area Under the Curve 
(Test Result Variable: Predicted Probability) ........................ 133

Table 4-17. Results of Model Validation with the 50\% Hold-Out Sample $\ldots . . . . . . .134$

Table 4-18. Prediction Power of the Result

Model Based on the Hold-out Sample ................................ 135

Table 4-19. Overall and Categorized Risk

Distributions in the Study Control Sample $\quad . . . . . . . . . . . . . . . . . . . . . . . .139$

Table 4-20. Results of Cost-Effectiveness Analysis Using Mean Values ............. 141

Table 4-21. Sensitivity Analysis on AE probability and WTC Rate $\ldots . \ldots . . . . . . . .145$

Table 4-22. Cost-Effectiveness Ratios of the Three

Drug Therapies in the Whole Study Sample $\ldots . . . . . . . . . . . . . . . . . . . .150$

Table 4-23. Cost-Effectiveness Ratios of the Three Drug

Therapies in Women with Different Risk Levels .................... 151 


\section{LIST OF FIGURES}

FIGURE

PAGE

Figure 3-1. The Structure of the Proposed Decision Tree $\quad \ldots \ldots \ldots \ldots \ldots \ldots \ldots \ldots \ldots \ldots \ldots . . \ldots 75$

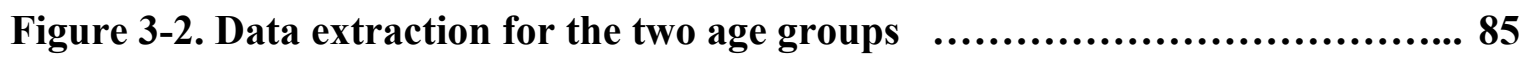

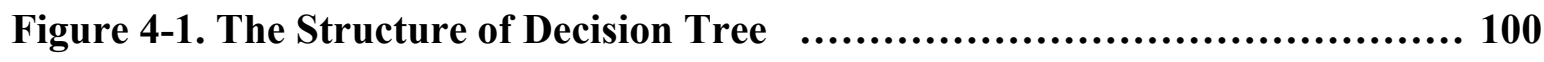

Figure 4-2. Results of Data Extraction for the Two Age Groups .................... 117

Figure 4-3. Results of Data Screening for Cases and Control Groups .............. 119

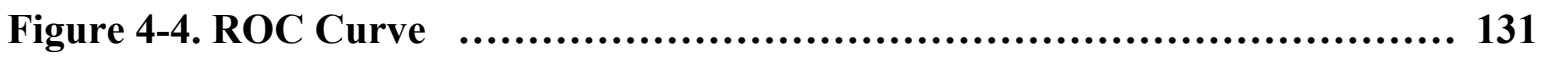

Figure 4-5. Risk Distribution in the Study Control Sample $(\mathrm{N}=390) \quad \ldots \ldots \ldots \ldots . .138$

Figure 4-6. Sensitivity Analysis on Treatment Costs ............................ 143

Figure 4-7. Sensitivity Analysis on Risk Reduction Rate $\quad$.......................... 144

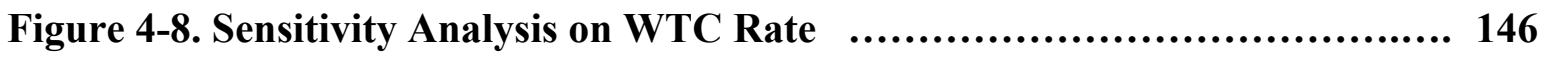

Figure 4-9. Decision Tree Structure Using Distribution Variables $\quad$ …............. 149 


\section{CHAPTER 1}

\section{INTRODUCTION}

\section{Epidemiology and Costs of Osteoporosis and Osteoporotic Fracture}

Osteoporosis is a systemic skeletal disease characterized by low bone mineral density (BMD) and microarchitectural deterioration of bone tissue, leading to bone fragility and increased susceptibility of fractures (Consensus Development Conference, 1991). It is especially prevalent in older postmenopausal women (Consensus Development Conference, 1991; Melton, 1995; Kanis et al., 1991). Because the age of onset of menopause is about 50, women can now expect to live one-third of their 80 -year life-span at risk for developing osteoporosis and fractures. The National Osteoporosis Foundation (NOF) has estimated that in 1996, approximately 29 million people aged 50 and over in the United States either had osteoporosis or were at risk of developing the disease (WV Bureau for Public Health, 1998). Incidence rate increases with age: 22\% among women 60 to 69 years old, $30 \%$ among women 70 to 79 years, and $70 \%$ among women 80 years or older (Prestwood \& Weksler, 1997). As a major cause of morbidity and mortality in postmenopausal women, osteoporosis induces a high burden of pain, physical disability, and has a profound effect on the quality of life among these women.

Osteoporosis is only clinically important when fractures occur (Lindsay \& Cosman, 1990). One of the most important clinical and economic implications of osteoporosis to society is the increased risk of bone fracture resulting from decreased BMD. The mortality rate in women from osteoporotic fracture is greater than the combined mortality rates from cancers of the uterus, cervix, and breast (Whittington \& 
Faulds, 1994). In the United States, approximately 250,000 hip fractures and 500,000 vertebral fractures are thought to occur each year in postmenopausal women (Prestwood \& Weksler, 1997; Lindsay \& Cosman, 1990). The cumulative lifetime fracture risks for a 50 -year-old woman are $40 \%$ for any fracture, $18 \%$ for hip, and $16 \%$ for spine (Kanis et al., 1994; Riggs \& Melton, 1995). Other studies indicated that the average lifetime risk of fractures for white women includes a $16 \%$ risk of one or more painful vertebral fracture, $15 \%$ risk of Colles' (wrist) fracture, and 16\% risk of hip fracture (Melton et al., 1993; Lauritzen et al., 1993). In addition, fracture rate increases by $30 \%$ for every decade of life (Gallagher, 1992). In 1995, \$13.8 billion in direct medical costs were spent on osteoporotic fractures (Ray et al., 1997).

The most common fractures consequent upon osteoporosis are fractures of hip, vertebral, and wrist (Colles' fracture) (Lindsay \& Cosman, 1990; Meunier et al., 1999). Although all of them are debilitating to patients, hip fracture is the most serious and costly. Women with hip fracture are 2 to 4 times more likely to die within 12 months of the event as are women of the same age in the general population without hip fracture (Schurch et al., 1996). Hip fracture is reported to result in about 30,000 deaths each year in the USA (Lindsay \& Cosman, 1990). The average mortality rate is $12 \%$ to $36 \%$ within one year (Egol et al., 1997). In women surviving one year after a hip fracture, high morbidity rate is observed (Marotolli et al., 1992; Cummings et al., 1988; Magaziner et al., 1990; Mossey et al., 1989; Jalovaara \& Virkkunen, 1991). Half of all hip fracture patients will be unable to live independently. In a prospective study, $50 \%, 70 \%$, and $87 \%$ 
did not recover the ability to independently walk, transfer from one place to another, and climb stairs, respectively (Marotolli et al., 1992).

Although hip fractures represent only a small part $(<10 \%)$ of the fractures, the financial cost of hip fracture is enormous. Hip fractures are responsible for more hospital bed days among women after age 45 than are myocardial infarction, breast cancer, chronic obstructive pulmonary disease, or diabetes (Kanis et al, 1997). About $35 \%$ of patients with limb fractures require hospital admission, including all patients with hip fracture, who may require up to 33 days in an acute setting (Lindsay \& Cosman, 1990). The total costs (including direct and indirect costs) of hip fractures were estimated to be \$8.7 billion in the USA in 1988 (Melton, 1993). Those costs can only be expected to increase as the proportion of elderly increases in the population.

Vertebral fracture is the most common osteoporotic fracture and usually occurs earlier in life than hip fracture. The prevalence of vertebral fractures among the aging population may exceed $75 \%$ for very aged women (Lindsay \& Cosman, 1990). Like hip fracture, vertebral fracture is associated with considerable morbidity (Gold, 1996; Ross, 1997). It may be painless and gradually progressive for years, or acute and accompanied by severe back pain. Among women with symptomatic vertebral fracture and chronic pain, $60 \%$ to $87 \%$ reported problems with carrying, lifting, walking, doing housework, and shopping (Cook et al., 1993). Although about two thirds of all vertebral fractures are not recognized clinically, both diagnosed and undiagnosed vertebral fractures are associated with pain and impaired physical function (Huang et al., 1996; Nevitt et al., 1998). True incidence rates have been difficult to determine due to the insidious onset of 
these fractures. Estimates of incidence based on the prevalence data available show that incidence begins to increase around the time of menopause and continues to rise throughout life (Lindsay \& Cosman, 1990; Melton et al., 1993).

Vertebral fractures, although causing pain and loss of height, tend to require a relatively small proportion of healthcare resources. Hospitalization, if required, is generally brief. Assuming that only $50 \%$ of fractures would require treatment and no costs for the remaining $50 \%$, the direct and indirect costs were estimated ranging from $\$ 8,723$ to $\$ 9,917$ (1988 dollars) per vertebral fracture depending on patient age (Clark and Schuttinga, 1992). The total cost per vertebral fracture was approximately $25 \%$ of the estimated cost of a hip fracture.

Wrist fracture may represent the earliest effect of osteoporosis. The incidence of wrist fracture increases rapidly around menopause (Melton, 1993). Wrist fractures are usually treated on an outpatient basis. Only $8 \%$ to $10 \%$ of patients experiencing wrist fracture are hospitalized, although this rate may increase to $76 \%$ after age 85 (Melton, 1993; Chrischilles et al., 1994). A severe consequence of wrist fracture is algodystrophy (reflex sympathetic dystrophy): less than $10 \%$ of patients were affected in most surveys, but the number has been reported to be as high as 30\% (Kanis \&Pitt, 1992). The costs of wrist fractures are typically associated with a number of follow-up physician visits. A total cost of $\$ 250$ per wrist fracture (1982 dollars) was estimated, but no detailed cost components were provided (Weinstein \& Schiff, 1983). 
Fracture of any bone can occur as part of the osteoporotic syndrome. After the commonest fractures mentioned above, other fractures encountered are of the proximal humerus, pelvis, and proximal tibia (Riggs \& Melton, 1986).

Factors Associated with the Development of Osteoporosis and Risk of Fracture

Various risk factors for osteoporosis and osteoporotic fractures have been presented in literature, although some of them are debatable. So far, studies in all populations show that the incidence of osteoporotic fractures increases with age. Bone mass is found to decrease with age in all studies. The relationship between low bone mineral density (BMD) and fracture occurrence has been well established (e.g., Ross et al., 1988 ${ }^{1,2}$; Lindsay \& Cosman, 1990). Women with very low BMD have much higher risks than the average, and many of them will experience fractures if untreated. Hence, it is not surprising that the major risk factors are advanced age and low BMD.

Among women, postmenopausal hormonal changes are also related to their BMD level and thus osteoporosis risk. Women with menopause-related estrogen deficiency are found to be at high risk for osteoporosis and fractures (Lindsay \& Cosman, 1990; Willhite, 1998). Other factors that increase risk include being of Caucasian or Asian race, thin body frame or underweight, early age at menopause (either natural or surgically induced), family history, and inadequate calcium and vitamin D intake. Sedentary lifestyle, smoking, and excessive alcohol and caffeine consumption have also been linked with bone loss, although the associations are not strong (Osteoporosis Task Force, 1996; Bauer et al., 1993; Adachi 1993; Lydick et al., 1998; Johnell et al., 1995). Evidence for 
increased risk by hyperathyroidism, thyrotoxicosis, and long-acting psychotropic drugs is controversial and needs further evaluation (Odell \& Heath, 1993; Reid, 1998).

Furthermore, many studies show that women who have one osteoporotic fracture are more likely to experience further fractures. Consequently, prior fracture itself is an important predictor for future fracture (Ross et al., 1991; Ross et al., 1993; Ensrud et al., 1997).

\section{Osteoporosis in West Virginia (WV)}

Osteoporosis has become a serious public health issue in WV. As mentioned above, age, sex, and race all play an important role in the development of osteoporosis, with older white women at high risk for the disease. In 1996, WV had the oldest population in the nation, with a median age of 38 , and ranked fourth in the U.S. in the proportion of its population that is elderly $(\geq 65)$. Also, women compose a larger percentage of the population in WV than in the nation as a whole: $53.1 \%$ vs. $51.3 \%$ in 1996. Finally, WV has a very small minority population, with more than $96 \%$ of the population classified as white.

In WV, a total of 183,962 women suffered from either low bone mass or osteoporosis in 1996. Additionally, 416 out of every 1,000 people aged 50 and older in WV either already had osteoporosis or were at risk. Approximately six out of every ten women in this age group were at risk for osteoporosis or osteoporotic fractures (WV Bureau for Public Health, 1998). It is predicted that if no action is taken, a total of 227,246 women in WV will have osteoporosis or low bone mass by 2015 (National Osteoporosis Foundation, 1996). Among state women aged 45 years and older, the 
incidence of all fractures will increase by 19\% from 1995 through 2015, more than 41,000 women will experience hip fractures and result in about 5,200 deaths, as well as approximately 100,000 and 38,500 will experience vertebral and wrist fractures respectively (Abbott et al., 1996)

The estimated hospitalization costs related to osteoporotic fractures in 1996 exceeded $\$ 42$ million, of which an estimated $\$ 36$ million were billed to Medicare. Women accounted for approximately four out of five (81\%) osteoporotic fracture hospitalizations, with an average stay of 8.83 days. The average charge per osteoporotic fracture among female patients was $\$ 10,219$ of which two-thirds was for hip fractures. Given the aging of West Virginia's population, the public health burden of this disease will become worse if no effective action is taken (WV Bureau for Public Health, 1998).

\section{$\underline{\text { Prevention Strategies }}$}

Osteoporosis is a complex chronic disease that may progress silently for many years until a fracture occurs. Patient management is difficult because many patients with osteoporosis are asymptomatic. Most patients are not diagnosed in time for effective prevention or treatment. If untreated, more than half of white women will experience at least one osteoporotic fracture during their lifetimes (Jones et al., 1994; Ross, 1996; Cooper, 1997). Although effective treatment after the first fracture presents can substantially reduce the risk of further fractures, the risk remains appreciable. Also, patients' quality of life is reduced irreversibly due to continuing pain and impaired mobility from the first fracture. It is preferable to intervene early in the process of bone 
loss and thus reduce the risk of the first fracture. As suggested earlier, preventing the first fracture can dramatically decrease the risk of further fractures.

Preventive strategies are essential for reducing the incidence of osteoporosis and consequent fractures. Studies have shown that recommending preventive therapy for all menopausal women is not appropriate (Lindsay \& Cosman, 1990; Riggs \& Melton, 1986; JÖnsson, 1998). Nevertheless menopause, because of its association with acceleration of bone loss, is probably a useful time at which to evaluate women for their risk of osteoporosis or osteoporotic fractures and to target treatment for high risk women. Costeffectiveness analyses of the "high-risk approach" suggest economic benefits of policies targeting pharmacological treatments to women at high risk (Jönsson, 1998).

Bone density is a good predictor of future fracture risk. In clinical practice, BMD testing plays an important role in the detection and management of osteoporosis and fractures. BMD test can be used to select individuals for osteoporosis/osteoporotic fracture prevention and treatment and to monitor response to therapy. In practice, $\mathrm{T}$ scores (standard deviations below peak bone density) are often used to represent risk of osteoporosis and osteoporotic fractures. However, the bone mineral measurement procedure is "too expensive and time-consuming for general use in the unselected population" (Michaelsson et al., 1996). There is a great need for simple means of identifying osteoporosis/osteoporotic fractures risk in population-based studies. Several important risk factors have been used to evaluate patients' fracture risk through a BMD prediction model. Those include age, age at menopause (or years since menopause), and weight (Carroll et al., 1997). Although using risk factors as an indirect way to estimate 
patients' fracture risk may not be accurate enough to replace the BMD test in clinical practice, it may still be reliable and applicable for population-based studies (e.g., Michaelsson et al., 1996; Kroger et al.,1994; Ribot et al., 1992).

In brief, although treatment options exist, the most effective way of dealing with osteoporosis is prevention aimed at high risk population. For middle-aged and older individuals, modification of risk factors (e.g., changing diet and lifestyle by taking calcium supplementation and doing weight-bearing exercise) and drug therapies are the major forms of prevention strategies for osteoporotic fractures. Regarding preventive drug therapy, there are three main intervention strategies available for preventing and treating osteoporosis/osteoporotic fracture: Hormonal replacement therapy (HRT), Alendronate therapy, and Raloxifene therapy.

Hormonal Replacement Therapy. HRT is the most commonly used method for osteoporosis prevention and treatment, as well as the treatment of menopausal symptoms. Studies have indicated that HRT substantially increases BMD and reduces fracture risk (e.g., Weiss et al., 1980; Ettinger et al., 1985; Keil et al., 1987; Felson et al., 1993). The additional benefits associated with HRT include the reduced risk of cardiovascular disease and Alzheimer's disease, relief from the symptoms of estrogen deficiency such as hot flashes, and a decrease in urogenital effects (Adis International Limited, 1998; Cooper, 1997).

The major risk of unopposed estrogen therapy is endometrial cancer. The reported magnitude of relative risk has varied from 1.7 to 20 times (Heidrich \& Thompson, 1987). However, concomitant use of estrogen with a progestin in HRT for 
women with an intact uterus minimizes the increased risk of endometrial cancer. The association between HRT and breast cancer has not been definitively established. The data are conflicting and the relative risk is between 1.0 and 2.0 (Collaborative Group on Hormonal Factors in Breast Cancer, 1997; Tosteson, 1994; DeGregorio \& Taras, 1998). The use of estrogen is also reported to slightly increase risk of venous thromboembolic events (DeGregorio \& Taras, 1998; Meunier et al., 1999). Other major adverse effects include breast pain, vaginal bleeding, headache, fluid retention, and GI complaints (e.g., nausea, vomiting, and cramps). Although side effects can sometimes be alleviated by changing the treatment regimen, some women cannot tolerate estrogen or HRT. Alendronate Therapy. Alendronate, a bisphosphonate, inhibits osteoclasic bone resorption. Alendronate binds preferentially to active sites of bone resorption and is not biologically active; thus, unlike estrogen, its effect may last long after discontinuation. Also, unlike early bisphosphonates such as etidronate, alendronate does not suppress bone formation, and it can be given continuously. It is the only FDA approved bisphosphonate for the prevention and treatment of osteoporosis (Willhite, 1998). Numerous studies have demonstrated that Alendronate treatment effectively increases BMD in women aged 44 to 84 years. It significantly prevents bone loss in early postmenopausal women and reduces the risk of fracture at all sites (Karpf et al., 1997; Black et al., 1996). There are no reported additional benefits associated with Alendronate therapy. 
The most serious side effect of Alendronate is esophageal ulceration. Less serious, but more common adverse effects are gastrointestinal: abdominal pain, nausea, dyspepsia, constipation and diarrhea (Adis International Limited, 1998).

Raloxifene Therapy. Raloxifene is the first selective estrogen receptor modulator (SERM) to be approved for the prevention of osteoporosis in postmenopausal women. It prevents bone loss and significantly increases BMD at the lumbar spine, hip, femoral neck, and overall in postmenopausal women with or without osteoporosis (Balfour \& Goa, 1998). Although improvements in BMD may be greater with HRT than with raloxifene therapy in elder postmenopausal women, unlike unopposed estrogen and tamoxifen (the first SERM developed), raloxifene does not have endometrial stimulatory effects and therefore does not increase the risk of endometrial cancer (van Leeuwen et al., 1994). In addition, daily therapy with raloxifene lowers serum levels of total and lowdensity lipoprotein cholesterol. However, it is unclear whether this effect on lipids will translate into the clear cardiac benefit that HRT provides (Balfour \& Goa, 1998; Kushner, 1998).

Compared with continuous HRT, raloxifene appeared to cause less breast pain, vaginal bleeding, flatulence, and abdominal pain. However, unlike estrogen, raloxifene does not relieve menopausal symptoms such as hot flushes. Raloxifene's potential for causing leg cramps and venous thrombosis is similar to that of estrogen (Kushner, 1998; Eli Lilly and Company, 1997). 


\section{Need for Study}

The growth rate in health care spending has occurred at more than twice the general inflation rate for two decades in the U.S. (ACCP, 1997). Spending of both private and government health insurance programs (i.e., Medicaid and Medicare) has significantly increased. In order to curtail growth in health care expenditures and utilization, numerous nationwide policy interventions have been attempted over the past two decades. The major interventions include capitation, prospective payment system, drug formularies, and physician payment reforms. Those cost containment strategies make third-party payers/insurers take both a financial risk and responsibility for health service delivery. Health care providers are also given strong incentives for costconscious health care delivery under those policies. Although constraining health care cost has been driven largely by the private sector with employers seeking to slow the growth rates in health care premiums paid for their employees, federal and state governments that fund Medicaid and Medicare programs have also been playing very important roles in promoting cost control.

The national trend in health care cost is also evident in West Virginia (WV). The average per member costs for WV Medicaid have gone up from about $\$ 675$ in 1982 to about \$3,696 in 1999 (National Pharmaceutical Council, 1983; WV Medicaid HCFA 2082 Report, 1999). The drug costs percentage of total medical costs also increased from $8.3 \%$ in 1993 to $15.4 \%$ in 1999 , and the average drug cost per recipient rose from $\$ 335$ to \$712 during the same time period (National Pharmaceutical Council, 1983; WV Medicaid HCFA 2082 Report, 1999). The state's response to the rapid growth in health 
costs is the implementation of several cost-containment policies such as requiring a drug prior authorization process, increasing co-payment, reducing pharmacy reimbursements, and imposing a 10-prescription limit per month per member. For WV Medicaid, making every effort to control costs while maintaining health care quality has become increasingly important.

As mentioned before, osteoporosis/osteoporotic fractures is a serious problem in WV. Osteoporosis and its associated fractures are an important public health problem that will worsen as the population ages. The large number of postmenopausal women at risk and the high toll of osteoporotic fractures in regard to functional status and health care cost have motivated increased thirty-party payers' awareness. Although most hospitalization costs of fractures are born by Medicare, they also constitute significant Medicaid expenditures for pharmaceuticals. Since controlling costs is a priority of WV Medicaid, how to control the costs associated with osteoporotic fractures has become a major concern for Medicaid.

Under this circumstance, increased interest has been shown in detection and prevention of osteoporosis/osteoporotic fractures and determination of optimal preventive interventions. Regarding the prevention of osteoporotic fractures, most researchers and physicians recommend that treatment should be limited to those women at high risk of developing osteoporosis/osteoporotic fractures (e.g., Willhite, 1998; Jönsson, 1998). However, questions arise about how to identify women at different risk levels. Controversies exist regarding the evaluation for fracture risk. Although several studies have attempted to use various risk factors to predict BMD or incidence of osteoporotic 
fractures, the results were not satisfactory (Lydick et al., 1998; Carroll et al., 1997; Blalock et al., 1996; Franceschi et al., 1996). The sensitivity and/or specificity were low and most studies were limited by the relatively small sample size and the type of fractures. It is not clear which and how risk factor(s) should be measured to estimate and quantify the risk levels of osteoporotic fractures in the WV Medicaid population.

With respect to preventive drug therapies, the implementation of new therapies such as raloxifene and the existence of multiple drug therapy options have raised several questions for WV Medicaid. The raloxifene and alendronate therapies are much more expensive than the traditional HRT. In 1998, the average acquisition costs per year for raloxifene, alendronate, and HRT were about $\$ 594, \$ 642$, and $\$ 223$, respectively (Adis International Limited, 1998). In order to control the escalating drug expenditures, Medicaid needs to decide who should be covered for drug therapies to prevent osteoporotic fractures. Preventive drug therapy for all women may be cost-prohibitive, risky, and wasteful. Also, considering the benefits of HRT and raloxifene as well as their risks and side effects, the net economic and clinical consequences of these therapies are vital issues. In a cost conscious environment, the choice of a preventive strategy for any disease is highly dependent upon cost-effectiveness or cost-benefit considerations. In other words, in order to use health care resources efficiently, it is critical to consider both the costs and potential consequences of different prevention strategies.

Medication adherence is another key issue. A cost-effective therapy proven in the controlled settings of clinical trials may not be of the same effectiveness in the real world. It is important to find an approach reflecting the realities of women's choice in the levels 
of adherence achievable with each therapeutic intervention. Decisions regarding medication adherence depend on an individual woman's preferences, which may further rely on her understanding of the risk-benefit equation of a specific therapy. For example, in the case of HRT, discontinuation and noncompliance are major problems. Of women who do receive a prescription for HRT, nearly $30 \%$ never have it filled, $20 \%$ stop taking the medication within nine months (Ravnikar, 1987), and at least 25\% stop treatment within 2 years (Kushner, 1998). Perceived lack of need for HRT and concern for adverse effects (breast cancer and vaginal bleeding) are the main reasons for never using HRT or noncompliance (Wysowski et al., 1995; Salamone et al., 1996). Obviously, the effectiveness of HRT for non-adherents would be reduced. Therefore, the problem of non-adherence in medication taking behaviors should be considered in cost-effectiveness evaluations. Currently, little information is available on how medication adherence will influence the relative cost-effectiveness of HRT compared to alternative strategies.

Finally, the cost-effectiveness of an intervention may vary among women at different risk levels. For example, raloxifene may be cost-effective for women at high risk for osteoporotic fractures, but not for women at average or low risk. The usage of each therapy among women with different risks will determine the total possible costs to Medicaid for covering these preventive agents. Medicaid programs must determine which drug therapies are the most cost-effective at different risk levels to encourage their risk-appropriate utilization. Then the original question "who among Medicaid women should be covered for preventive drug therapies?" becomes "what are the costeffectiveness ratios of each therapy at different fracture risk levels?" In order to answer 
these questions, a decision model incorporating the costs and effectiveness of each therapy needs to be established and the most risk-appropriate therapy needs to be determined.

Currently, no comparisons of the three drug therapies have been reported that take into consideration their costs, preventive effectiveness, and discontinuation rates. Direct comparative trials of the effects of the three treatment strategies on fracture prevention have so far not been published. In addition, no study has linked women's fracture risks to the cost-effectiveness evaluation of the drug therapies.

\section{Research Questions}

Based on the need for the study and review of the literature, this study will try to answer the following research questions with respect to the WV Medicaid female population. Since all of them are exploratory questions, no hypotheses are proposed.

1. What are the costs and risk reduction rates related to the three drug therapies for preventing osteoporotic fractures?

2. What is the background fracture risk among women 45 year and older in the study population?

3. What is the expected cost-effectiveness of the three drug therapies based upon the risk distribution of the study population? 


\section{Study Goal and Scope}

The goal of this study is to evaluate the cost effectiveness of three drug therapies for preventing osteoporotic fractures and to assess the economic impact of risk-based utilization on Medicaid program costs. The three intervention strategies that will be evaluated are: Hormone Replacement Therapy (HRT), alendronate (Fosamax ${ }^{\circledR}$, and raloxifene $\left(\right.$ Evista $\left.^{\circledR}\right)$. The study population will consist of postmenopausal women who are enrolled in WV Medicaid and aged 45 to 85 years. The perspective of the WV Medicaid program will be taken for this study.

\section{Conceptual Framework}

To complete the study goals, different methodologies are required. Hence the study will be completed in three phases. Phase I will construct a hypothetical decision model to evaluate the cost effectiveness of the three drug therapies for preventing osteoporotic fractures. Phase II will assess the distribution of the background risk in the study population. And Phase III will combine the information obtained from the first two phases and calculate the cost-effectiveness ratios of the three drug therapies. The economic impact of risk-based utilization on the Medicaid program expenditures will also be evaluated. The rationale and conceptual framework of the three phases are described below.

\section{Phase I. Model Development}

To substantially reduce the population rate of osteoporosis and fractures, a preventive intervention would need to be safe and effective for long periods to be 
acceptable for use. Since each current drug intervention is associated with risks and benefits, how to balance the costs and effectiveness becomes a critical issue.

A decision tree will be constructed to identify the relevant costs and consequences of the three competing drug interventions. A hypothetical treatment model for a white postmenopausal woman aged 45 years or older and without past incidence of osteoporotic fractures will be established. The woman will be treated with one of the three treatment alternatives, and tracked over a period of 3 years. This length of intervention is chosen to coincide with the time period for which relevant clinical data from studies are available (Eli Lilly and Company, 1997; Carroll et al., 1997; Meunier et al., 1999; Rosner et al., 1998). The model follows the incidence of fracture and adverse events associated with each therapy over a three-year period. The woman's compliance with each drug therapy will also be incorporated in the model.

Treatment effectiveness will be measured in terms of the number of fractures averted by each drug therapy. Resource costs will include the direct medication costs of each treatment strategy $\left(\mathrm{C}_{\text {Medication }}\right)$, monitoring costs $\left(\mathrm{C}_{\text {Monitoring }}\right)$, and the cost of the adverse effects $\left(\mathrm{C}_{\mathrm{AE}}\right)$. The cost-effectiveness ratios are thus defined as:

$$
\begin{aligned}
\mathrm{C} / \mathrm{E} & =\left(\mathrm{C}_{\text {Medication }}+\mathrm{C}_{\text {Monitoring }}+\mathrm{C}_{\mathrm{AE}}\right) /(\text { Number of Fractures Averted }) \\
& =\left(\mathrm{C}_{\text {Medication }}+\mathrm{C}_{\text {Monitoring }}+\mathrm{C}_{\mathrm{AE}}\right) /\left(\mathrm{I}_{0} * \mathrm{R}\right)
\end{aligned}
$$

The number of fracture prevented can be calculated as $\mathrm{I}_{0} * \mathrm{R}$, in which $\mathrm{I}_{0}$ was the background risk; and $\mathrm{R}$ was the risk reduction rate by the treatment.

Phase I will identify the $\mathrm{C}_{\text {Medication, }} \mathrm{C}_{\text {Monitoring, }}$, and $\mathrm{C}_{\mathrm{AE}}$, as well as the risk reduction rate and women's discontinuation rate of each treatment. However, for the background 
risk used for "no therapy" $\left(\mathrm{I}_{0}\right)$, a different approach is needed. Therefore, Phase II study is designed to obtain the background risk in the study population.

\section{Phase II. Analysis of Background Risk}

Literature shows that one way to achieve cost-effectiveness of preventive interventions is to target interventions at the high-risk population (Jönsson, 1998). The effectiveness of a preventive drug therapy tends to be more obvious or stronger in high risk population than that in low risk population. Consequently, the ratio of costs to risk reduction (effectiveness) of a drug therapy will be affected by the risk levels of the population. For example, a drug therapy unable to significantly reduce the number of fractures (ineffective in prevention) may be only due to the originally low fracture rate ("floor effect"). Unless the drug therapy is very cheap and/or has extra benefits other than preventing fractures, it is difficult for the small prevention effects to offset the costs in low-risk population. Therefore, uniform treatments prescribed for all postmenopausal women are unrealistic. The direct medical costs would increase dramatically, even though some people may argue the savings in long-term costs are not clear.

On the other hand, physicians and patients may be reluctant to use drug therapies in the absence of indications or symptoms. Since osteoporosis can be developed asymptomatically before a fracture occurs, this may lead to inadequate preventive care for women at high risk. To avoid the two situations in which women with low risk of the disease are "over-treated" and women with high risk are "under-treated", it is critical to identify women's risk levels for osteoporotic fractures and evaluate preventive drug 
therapies based on their risk. Then, an appropriate cost-effective drug therapy can be chosen for each woman based upon the most cost-effective drug available for their risk levels.

In Phase II, a regression model will be built by comparing the risk factors between women without previous osteoporotic fractures (controls) and those who had fracture experience after 45 (cases) to predict the risk of osteoporotic fractures. The risk factors examined in the model include: a) age; b) age at menopause; c) current Body Mass Index (BMI) and BMI in the 20s to 30s of age; d) family history; e) calcium and vitamin D intake; f) smoking behavior; g) alcohol consumption, and h) exercise. Since more than $96 \%$ of WV population are white, race will not be included in the model.

The established prediction model will then be applied to the sample which only contains fracture-free women to estimate their fracture probability. The predicted probability $(\mathrm{P})$ of fracture occurrence in the target fracture-free sample can be calculated by the following formulas (Wright, 1995):

$$
\begin{aligned}
& \mathrm{g}=\mathrm{b}_{0}+\mathrm{b}_{1}\left(\mathrm{x}_{1}\right)+\mathrm{b}_{2}\left(\mathrm{x}_{2}\right)+\mathrm{b}_{3}\left(\mathrm{x}_{3}\right) \ldots \\
& \mathrm{P}=\mathrm{e}^{\mathrm{g}} /\left(1+\mathrm{e}^{\mathrm{g}}\right)
\end{aligned}
$$

For each woman in the fracture-free sample, her probability of developing a osteoporotic fracture can be estimated by the model. The fracture probability distribution (Mean, SD) of the sample can then be inferred to the fracture-free women in the study population. 


\section{Phase III. Cost-Effectiveness Analysis}

Phase III of the study will evaluate the cost-effectiveness of the three drug therapies for women with different risk levels, utilizing the information obtained from Phase I and Phase II. A cost-effectiveness analysis will be conducted to determine which drug therapy is of the most value for the hypothetical woman in the decision-making model established in Phase I. As mentioned before, the effectiveness of treatments varies with the risk levels in the study population. This characteristic of uncertainty in the effectiveness value should be considered and reflected in the calculation of costeffectiveness ratios. To incorporate uncertainties in the effectiveness variable as well as the most influential cost variables to the evaluation (detected by simple sensitivity analyses), a probabilistic analysis (Monte Carlo simulations) will be conducted based on the distribution of background fracture risk $\left(\mathrm{I}_{0}\right)$, treatment cost, and fracture risk reduction rate. The expected values (expected costs per fracture averted per woman) will be estimated for the three preventive drug therapies among fracture-free women in the study population.

Using the hypothetical model and decision tree structure developed in Phase I, the base formula to calculate $\mathrm{CE}$ ratio of the Monte Carlo simulations will be:

$$
f[\operatorname{costs} /(\text { fracture averted })]=f(\mathrm{C}) /\left[\left(f\left(\mathrm{I}_{0}\right) \times f(\mathrm{R})\right]\right.
$$

in which $f(\mathrm{C})$ is the distribution of treatment costs for the treatment, $f\left(\mathrm{I}_{0}\right)$ is the distribution of background fracture risks, and $f(\mathrm{R})$ is the distribution of fracture risk reduction rates. $f\left(\mathrm{I}_{0}\right)$ will be estimated from the actual risk distribution in the study population obtained from Phase II. The distributions of treatment costs and fracture 
reduction rates will be estimated by Triangle distribution using high, basic, and low values.

Incremental cost-effectiveness ratio will be calculated for each treatment strategy relative to the next most effective strategy. Since it is well accepted that women who have high risk of osteoporosis should be treated for preventing osteoporotic fractures, the cost-effectiveness of each drug therapy relative to not using any intervention will be obvious. The question then turns to whether the additional benefit is worth the additional cost among the competing alternatives. Thus, it is important to compare incremental cost-effectiveness of alternative interventions.

Finally, a matrix of expected CE ratios by risk levels and by preventive drug strategies will be created to illustrate the potential economic and clinical outcomes. Recommendations on the optimal drug utilization and coverage can be made to the WV Medicaid Program.

\section{Study Objectives}

\section{Objective 1}

To develop a decision model that identifies the costs and effectiveness of three preventive drug therapies for osteoporotic fractures in postmenopausal women aged 45 to 85 years old.

\section{Objective 2}

To assess the relationship between various risk factors and the occurrence of first osteoporotic fracture in the study population. 


\section{$\underline{\text { Objective } 3}$}

To determine the distribution of women on the basis of their probabilities for osteoporotic fractures in the study population.

\section{Objective 4}

To examine the expected cost-effectiveness of each of the three drug therapies based on the fracture risk distribution in the study population.

\section{Research Significance}

One of the most important trends in health care is the increasing emphasis toward disease prevention. Besides the efforts aimed at adjusting behavioral factors, more drugs aimed at both primary and secondary prevention have been developed. However, the health care system nationwide has been plagued by escalating drug product costs. The introduction of new chemo-preventive agents may further threaten drug budgets of thirdparty payers, especially if this trend continues or escalates. The question arises which and how preventive medication(s) should be covered by third-party payers such as Medicaid, managed care organizations, and other insurance payers among their members with different risks for the disease.

A novel aspect of this study is the establishment of a risk-based approach to evaluate preventive drug therapies and make appropriate coverage decision. A drug therapy may not be cost-effective among low risk women, even if it is cost-effective among high risk women. Consequently, an evaluation of the cost-effectiveness of three drug therapies is more relevant and practical if done in women with different risks. For 
the WV Medicaid program, information regarding the costs per fracture averted based on the possible treatment costs and effectiveness as well as the fracture risk distribution can provide decision makers a better understanding of the possible expenditures and risks (uncertainty) associated with each prevention strategy. Findings of this study will also help policy makers assess the economic and clinical benefits of providing coverage for the drug therapies in women with different risk levels. Policy makers so informed can determine what drugs should be covered and who can receive them to optimize outcomes given the budget constraints.

The study will also have relevance to clinical decision-making. In clinical practice, physicians and other decision makers should consider the benefits, risks, and costs of each drug therapy as it applies to the individual patient. Information from a thorough evaluation of alternative therapies is greatly needed. This study will help decision makers to determine whether the costs associated with each therapy are reasonable or within accepted limits when both costs and benefits are taken into account. By comparing incremental cost-effectiveness of three drug therapies based on the women's risk, decision makers can choose the most cost-effective treatment for women at different risk levels. The established model can be used in the process of making formulary decision and developing clinical practice guidelines to improve and standardize preventive health care delivery.

Furthermore, the risk prediction model obtained in this study can be used to develop a risk assessment algorithm that clinicians can utilize to estimate fracture risk for each woman, and identify high-risk women either for an appropriate therapy or more 
costly advanced test (i.e., BMD test). A clinical guideline can also be developed based on the matrix of cost-effectiveness ratios by therapy and by fracture risk.

Finally, the methodology developed for assessing cost-effectiveness of preventive drug therapy will be an addition to the literature that is currently lacking in this important area of need. The risk distribution-based evaluation method will not only be useful to Medicaid programs but also to managed care organizations who also face similar cost concerns from new preventive chemo-therapies. 


\section{CHAPTER 2}

\section{LITERATURE REVIEW}

This chapter summarizes the results of an extensive review of the available literature regarding osteoporosis/osteoporotic fracture and its prevention and treatment, pharmacoeconomic evaluation of the three pharmacotherapies, fracture risk assessment, and the West Virginia Medicaid program.

\section{Postmenopausal Osteoporosis and Related Fractures}

\section{Burden of Illness}

Osteoporosis is a systemic bone disease associated with altered bone architecture, reduced bone mass, and an increased fracture rate. The World Health Organization has defined osteoporosis as a bone mineral-density measurement (T-score) of more than 2.5 standard deviations (SDs) below the mean for young adults. Bone densitometry reports refer to $\mathrm{Z}$ scores or $\mathrm{T}$ scores. The $\mathrm{Z}$ score (age-matched control) compares the patient with a population adjusted for age, sex, and weight; the T score (young, normal control) compares the patient with a sex-adjusted population at peak bone mass (Consensus Development Conference, 1991). A bone will be considered to be osteoporotic if it has sufficiently decreased density measured by a $\mathrm{T}$ or $\mathrm{Z}$ score, or if there has already been spontaneous or nontraumatic fracture. 
Osteoporosis is a major health and economic problem. The National Osteoporosis Foundation estimated that in 1996 approximately 29 million people aged 50 and over in the United States either had osteoporosis or were at risk of developing the disease; this number is expected to rise to over 41 million by the year 2015 (WV Bureau for Public Health, 1998).

Postmenopausal osteoporosis is the most common type of osteoporosis, compared to senile and secondary osteoporosis (caused by certain medications and/or diseases). Age-related bone loss commences in both men and women at about 40 years of age and continues throughout life, with acceleration in the rate of loss in women during menopause. The average age of onset of menopause in the US is 52 years (Hormone Replacement Therapy Technical Bulletin, 1992). Postmenopausal osteoporosis is usually diagnosed in women 51 to 75 years of age. The reduction in estrogen levels associated with menopause is an important contributory factor to bone mineral loss and the development of osteoporosis. Estrogen deficiency not only decreases protective effects on serum lipid profiles and other cardiovascular parameters, causing an increased risk of ischemic heart disease, but also results in increased bone resorption without change in bone formation, particularly in trabecular bone (Whittington \& Faulds, 1994). This leads postmenopausal women at high risk of fracture. A report from National Institutes of Health (NIH) shows that one in every two women will suffer an osteoporotic fracture at some time in their lives. A woman's risk of a hip fracture is equal to her COMBINED risk of having breast, uterine, or ovarian cancer (WV Bureau for Public Health, 1998). It is estimated that by the end of year 2000, there will be more than 50 million American 
women older than 50 years of age (Mosca et al., 1997). This suggests a large number of women who will be at risk of osteoporosis and would need appropriate preventive treatment. At present, most of the drugs used in the management of osteoporosis are approved only for the prevention and treatment of postmenopausal osteoporosis; other forms of the disease, such as corticosteroid-induced osteoporosis and osteoporosis in men, have not been studied well due to their relatively low prevalence.

The most serious consequence of osteoporosis is fracture. The National Osteoporosis Foundation (NOF) estimates that approximately 1.5 million fractures per year are osteoporosis related: 300,000 hip fractures, 700,000 vertebral fractures, 250,000 forearm fractures, and 250,000 fractures at other sites (National Osteoporosis Foundation, 1997). Osteoporotic fractures are a major cause of morbidity and mortality in elderly population. For instance, half of all hip fracture patients will be unable to live independently, $12 \%$ to $36 \%$ will die within one year, and the mortality significantly increases with age (Egol et al., 1997). One-half of persons who have a hip fracture will be unable to walk without assistance during their remaining lifetime, and 25\% will require long-term care (National Institute of Arthritis and Musculoskeletal and Skin Diseases, 1997). Osteoporosis can also cause kyphosis (dowager's hump) which contributes to the compression of internal organs and related consequences. Other effects of osteoporosis include decreased height, reduced lung capacity, and chronic pain (Prestwood \& Weksler, 1997). Patients' quality of life can also be decreased significantly by a functional disability, restricted activities, fear of falling, and the pain associated with osteoporosis, osteoporotic fractures, or kyphosis. 
The economic costs of treating osteoporosis and related fractures are enormous, both to individual patients and to society in general. Ray et al. (1996) estimated the direct costs to the health care system to be nearly $\$ 14$ billion annually or $\$ 38$ million each day. By 2040, the total annual cost of hip fractures alone is predicted to be $\$ 240$ billion (Cummings et al., 1990 ${ }^{1}$. The total societal costs may be underestimated since some indirect costs such as workday loss of care givers have not been considered.

As the number of elderly people in the US and the world increases, osteoporosis and its related fractures have become a focus of attention to the public. Considering the high prevalence of osteoporosis and its impact on health care expenditure and patients' quality of life, consideration of more vigorous prevention strategies is warranted.

\section{Prevalence and Incidence in West Virginia}

Because osteoporosis primarily affects older, white women, it becomes a particular concern in West Virginia. According to the U.S. Census Bureau, West Virginia ranks fourth in the nation in the proportion of its population that is elderly and has the highest median age in the nation, 37.7 years. White women also compose a larger percentage of the population in West Virginia than in the nation as a whole (Hobbs, 1996).

Data from the 1997 Behavioral Risk Factor Surveillance System (BRFSS), a CDC funded annual telephone survey of West Virginians aged 18 and older that monitors health behaviors, provided information on the prevalence of eight risk factors for osteoporosis: bone structure/body weight, menopause, heredity, thyroid or cortisone-like medications, consumption of dairy products, cigarette smoking, physical inactivity, and 
alcohol abuse. Specifically, $23.5 \%$ postmenopausal women smoked, $70.3 \%$ were sedentary, $90.0 \%$ consumed fewer than three servings of dairy products per day, and $19.0 \%$ had a family history of osteoporosis. Nearly nine out of every ten postmenopausal women $(87.7 \%)$ reported having three or more risk factors for osteoporosis (WV Bureau for Public Health, 1998).

Prevalence of Osteoporosis. Based on research summaries from the National Health and Nutrition Examination Survey III (NHANES III) conducted from 1988 through 1994, the National Osteoporosis Foundation (NOF) reported that in 1996 approximately $13 \%-14 \%$ of the men and women aged 50 and over in any given state had osteoporosis. The exceptions to this were the District of Columbia which had the lowest prevalence rate of $10 \%$, and West Virginia, Iowa, Maine, Nebraska, and Rhode Island had the highest prevalence rate of $15 \%$. The NOF study also estimated that a total of 182,962 women in West Virginia suffered from either osteoporosis $(63,683$, or 210.2 per 1,000 women) or low bone mass (119,279, or 393.7 per 1,000 women). That means 210 out of every 1,000 women aged 50 and older in WV already had osteoporosis and 394 women were at risk due to low bone density. By 2015, the NOF predicts that a total of 227,246 women in the state will have osteoporosis or low bone mass.

Incidence of Osteoporotic Fractures. The state burden of fractures model developed by Merck and Company, Inc. (Abbott, 1996) predicted the number of fractures in women aged 45 and older in each of the 50 states and the District of Columbia annually from 1995 through the year 2015. It estimated that more than 41,000 women in WV would experience hip fractures during this period. Approximately 38,500 women 
would suffer a wrist fracture between 1995 and 2015, while nearly 100,000 women would experience vertebral fractures. Also, the model predicted that from 1995-2015 approximately 8,400 West Virginia women aged 50 and older would die within a year following a hip fracture. Of these, 5,200 deaths were attributable to the fracture itself.

The prevalence and incidence statistics in WV are alarming. With the aging of the population, the situation will worsen if no effective intervention strategy is done.

\section{Prevention and Treatment}

Currently, the management of osteoporosis includes both treatment and prevention. Patients with osteoporosis or osteoporotic fractures can be treated by HRT, bisphosphonates, calcitonin, or lifestyle modification (e.g., change in diet, smoking cessation, and exercise)(Umland et al., 1999). The primary goal of treatment in osteoporosis is to prevent fractures.

Although treatment options exist, the most effective method of dealing with osteoporosis and related fractures is prevention through lifestyle modification, increased calcium and vitamine D intake, and the use of pharmacotherapy such as estrogen, alendronate, or raloxifene. Preventing osteoporosis will ultimately prevent fractures. Too often the diagnosis of osteoporosis is made only after the occurrence of a fracture. An early identification and modification of a person's risk factors are therefore important to prevent osteoporotic fractures. Risk factors for osteoporosis include postmenopausal period, race (being white or Asian), thin body build, cigarette smoking, moderate to heavy alcohol use, sedentary lifestyle, diet deficient in calcium, and premature menopause (Consensus Development Conference, 1993). 
Several clinical guidelines for the prevention, treatment, and management of osteoporosis have been developed (e.g., Scientific Advisory Board, 1996; Osteoporosis Task Force, 1996; Kushner, 1998). All guidelines suggest that osteoporosis is preventable and treatable. Prevention and early treatment of osteoporosis are believed to be a key in limiting the progression of osteoporosis and therefore avoiding the fracture, which is a major public health problem. Appropriate treatment strategies, especially pharmacotherapies, can significantly avert or lessen the morbidity and mortality associated with fractures associated with the osteoporosis.

\section{Pharmacotherapy in the Treatment and Prevention of Osteoporosis}

Major changes in the prevention and treatment of osteoporosis and related fractures have occurred in the last three years with the approval of new drugs such as alendronate and raloxifene. These two new drugs can be used as an alternative to hormone replacement therapy, which has been used for many years. All of these therapies vary in their regimen, benefits, risks, and adverse effects. As with all therapeutic decisions, physicians should consider the benefits, risks, and costs of each treatment when applying it to the individual patient. The comparative efficacy of these agents have been discussed in many study reviews, especially for HRT and alendronate (e.g., Whittington \& Faulds, 1994; Meunier et al., 1999; Scientific Advisory Board, 1996; Willhite, 1998). Only a brief summary is presented here. 


\section{Hormone Replacement Therapy (HRT)}

\section{$\underline{\text { Regimens }}$}

All forms of estrogen (including transdermal, equine and synthetic estrogens) are effective in preventing bone loss (Scientific Advisory Board, 1996). Progestins are given along with estrogen therapy to prevent the development of endometrial hyperplasia and carcinoma. Two major hormone replacement schedules are used for women who have a uterus (Dosh et al., 1997). Estrogen and progesterone in combination can be taken either cyclically or continuously. Cyclical combined schedule more or less copies the natural menstrual cycle. Estrogen is taken on days one to twenty-five and progesterone is added on or about day fourteen. After day twenty-five, no hormone is taken for five days, during which time bleeding will probably occur. This bleeding gradually diminishes over the years and eventually disappears. Continuous combined schedule requires women to take both estrogen and progesterone every day. The lining of the uterus thins and never builds up, so there is no bleeding. However, because taking progesterone does not duplicate the cyclical biological schedule of the premenopausal woman, some researchers feel that it is less effective in protecting you against heart disease.

The most common dosage forms for the prevention and treatment of osteoporosis are: (1) cyclic estrogen $0.625 \mathrm{mg} /$ day + cyclic medroxyprogesterone $5 \mathrm{mg} /$ day; 2 ) continuous estrogen $0.625 \mathrm{mg} / \mathrm{day}+$ continuous Medroxyprogesterone 2.5mg/day; (3) cyclic transdermal estradiol 50 $\mathrm{g}$ /day + cyclic Medroxyprogesterone 5mg/day; and (4) Continuous transdermal estradio $150 \mu \mathrm{g} /$ day + continuous Medroxyprogesterone $2.5 \mathrm{mg} /$ day. 


\section{$\underline{\text { Effects on Bone Loss and Fractures }}$}

The protective effect of HRT on bone has been widely reported (Whittington \& Faulds, 1994; The Writing Group for the PEPI Trial, 1996; Mizunuma et al., 1997; Eiken et al., 1997; Schneider 1997; Lufkin et al., 1992). HRT is effective against osteoporosis in $85 \%$ of users and is the first line pharmacotherapy for prevention and treatment of osteoporosis in postmenopausal women (Scientific Advisory Board, 1996; Whittington \& Faulds, 1994; Willhite,1998; Meunier et al., 1999; Isenbarger et al., 1997). Estrogen decreases bone resorption by inhibiting osteoclastactivity and increases both cortical ( $1 \%$ to $3 \%$ ) and trabecular (2\% to $5 \%$ ) bone density (Osteoporosis Task Force, 1996). The total BMD can be increased up to $12.1 \%$. Estrogen therapy with or without concurrent progesterone therapy prevents accelerated bone loss that normally occurs with menopause. Combined estrogen and progestogen result in similar risk reductions to unopposed therapy.

Epidemiology studies have shown decreased osteoporotic fractures in HRT users (Miller, 1996). The Postmenopausal Estrogen/Progestin Intervention (PEPI) trial, a 3year, multicenter, randomized, double-blind, placebo-controlled study, examined the effects of ERT on BMD in postmenopausal women (The Writing Group for the PEPI Trial, 1996). It found that BMD increased by a mean of $5.1 \%$ in the spine and $2.3 \%$ in the hip in the treated group. Long-term therapy reduced the risk of hip fracture by $25 \%$.

A meta-analysis of 47 randomized controlled trials indicated that pooled RR rate was 0.5 for case-control and 0.84 for cohort studies (Robertson et al., 1993). Another comprehensive meta-analysis showed a $25 \%$ risk reduction $(R R=0.75)$ in osteoporotic 
hip fractures in ever-users of HRT compared with non-users from 11 epidemiology studies (Grady et al., 1992). Results also suggested that the relative risk would further decrease to 0.5 after $>10$ years of therapy. Longer duration of HRT may be associated with greater effect although the optimal duration is still unknown (Miller, 1996). To confirm its antiosteoporotic efficacy, BMD test after 2 years of treatment is recommended (Kushner, 1998).

Given the effects of HRT on bone loss and fracture risk, the purpose of using HRT in the prevention and treatment of osteoporosis is to provide the lowest and safest level of ovarian hormone dosage that will protect against osteoporotic fracture (Scientific Advisory Board, 1996).

\section{Effects on Cardiovascular Disease}

Cardiovascular disease is considered the most significant health problem. It is the number one cause of death in post-menopausal women (DeGregorio \& Taras, 1998). Many studies show that HRT has a protective effect against coronary heart disease in addition to its effect on preventing bone loss and fractures (Haines et al., 1996; The Writing Group for the PEPI Trial, 1995; Koh et al., 1999; Hulley et al., 1998). Results showed that HRT reduced low-density lipoprotein (LDL) levels by $15-19 \%$ and increased high-density lipoprotein (HDL) by $6-18 \%$. HRT in postmenopausal women decreases the risk of coronary heart disease through its effect on lipid profiles. Approximately $75 \%$ of estrogen's cardiovascular benefits come from vasodilation, antiplatelet effects, and other direct benefits on the arterial wall (Wild, 1996). 
The effect of estrogens on raising triglyceride levels may be offset by adding progestin. Although both ERT and HRT provide lipid benefits, concomitant progestin use may decrease estrogen's positive effects on serum lipid profiles (Whittington \& Faulds, 1994; Grady, 1992). However, this mechanism is still unclear and only partly responsible for the cardioprotective effect and depends on the type of progestogen used (Whittington \& Faulds, 1994).

Some studies also examined the association between ERT/HRT and the endpoint outcomes such as risk of cardiovascular disease and related morbidity and mortality (Grodstein et al., 1996; Grodstein et al., 1997). Nabulsi et al. (1993) used a crosssectional analysis and estimated a $42 \%$ reduction in the relative risk of coronary heart disease in HRT users compared with non-users. A large $(n=59,337), 16$-year follow-up in the Nurses' Health Study showed an approximately $60 \%$ decreased risk of major coronary artery disease for women on HRT or estrogen alone, compared with nonhormone users (Grodstein et al., 1996). However, large, randomized clinical trials are needed to evaluate the impact of HRT on heart disease risk as well as related morbidity and mortality.

\section{Other Benefits of HRT}

Estrogen can relieve menopause symptoms such as hot flashes, vaginal atrophy, and vaginal dryness (Miller, 1996). The role of estrogen in memory and Alzheimer's disease is not definitive (Henderson et al., 2000; Paganini-Hill, 1997; Paganini-Hill \& Henderson, 1996; Paganini-Hill, 1996). The Leisure World Cohort Study showed a relative risk of Alzheimer's disease or dementia in ERT ever-users of 0.69 compared to 
non-users (Paganini-Hill \& Henderson, 1996). But other studies have shown no benefit or an increased risk of dementia in estrogen users (Paganini-Hill, 1996).

The association between estrogen/progestogen use and stroke is unclear.

Evidence is controversial in the literature. For example, Grady et al. (1992) estimated a relative risk (RR) of 0.96 for stroke among estrogen users. Another two large cohort studies estimated the RR of any stroke to be 0.69 and 0.90 for estrogen users, and RR of stroke mortality as 0.37 (Finucane et al., 1993; Falkeborn et al., 1993). However, estrogen use did not shown any protective effect against stroke in the Nurses' HealthStudy - a large prospective trial (Grodstein et al., 1996).

\section{$\underline{\text { Risks and Adverse Effects of HRT }}$}

Breast Cancer. The relationship between HRT and breast cancer is complicated and highly controversial in literature. Many studies revealed no apparent increased risk of breast cancer in ever ERT/HRT users (Colditz et al., 1993; Grady et al., 1992). In contrast, some studies revealed that there is an increased risk of breast cancer in current HRT users, especially in long-term users, with relative risks (RR) ranging from 1.2 to 1.63 (Colditz et al., 1993; Sillero-Arenas et al., 1992; Collaborative Group on Hormonal Factors in Breast Cancer, 1997; Roy et al., 1996). For example, the Collaborative Group on Hormonal Factors in Breast Cancer reanalyzed data from 51 epidemiology studies of 52,705 women with breast cancer and 108,411 women without breast cancer (Collaborative Group on Hormonal Factors in Breast Cancer, 1997). In women who used ERT for 5 years or more, the RR of breast cancer was 1.35. In women who had never used estrogen, the RR rose by 1.03 for each year past menopause. The authors concluded 
that the risk of breast cancer increased with the duration of ERT use. However, the data also showed that the risk of breast cancer reverted to normal after 5 years of stopping ERT.

No relationship was found between the dose of estrogen and RR of breast cancer. Also, adding progestin to ERT did not reduce the overall relative risk of breast cancer (Colditz et al., 1993; Sillero-Arenas et al., 1992; Collaborative Group on Hormonal Factors in Breast Cancer, 1997). And interestingly, no increase in RR of breast cancer was seen among women with a family history of the disease, compared with those without a family history (Colditz et al., 1993).

Results and conclusions from many of those studies regarding the effect of HRT use on the risk of breast cancer were limited by a strong detection bias, because women on ERT/HRT tend to receive more thorough breast cancer screening. Also, many studies failed to control some key variables such as alcohol intake which may also influence the risk of breast cancer (Zumoff 1993). Large, prospective, randomized, and wellcontrolled trials are needed to establish any relationship between HRT and breast cancer.

Uterine Cancer. Unopposed estrogen use is related to endometrial cancer and this relationship has been well proved by many case-control and cohort studies (Grady et al, 1992). However, the risk of uterine cancer in women with an intact uterus can be virtually eliminated when estrogen is combined with progestogen. The conclusion has been well accepted by researchers and clinicians that the relative risk of endometrial cancer in women on HRT (estrogen + progenstogen) is the same as that in untreated 
women (e.g., Whittington \& Faulds, 1994; Willhite, 1998; Meunier et al., 1999; Grady et al., 1992).

Major Adverse Effects. The major side effect of HRT is irregular vaginal

bleeding. An increased risk of deep venous thromboembolism and pulmonary embolism is also associated with HRT. Studies found that the increased risk is only in current users and past users are not at increased risk for these adverse effects. Short-term current users appeared to have highest risk (Grodstein et al., 1996; Daly et al., 1996). The risk of venous thromboembolism doubled with HRT use, but due to the low baseline risk, HRT produced only one to two additional cases per 10,000 women per year (Gutthann et al., 1997). The risk of pulmonary embolism was also showed as twice as that of non-users (Grodstein et al., 1996) and had low incidence rate.

Other side effects of HRT are nausea, vomiting, abdominal cramps, mastalgia, bloating, hair loss, and headaches. The effects of HRT on blood pressure and glucose tolerance are not significant enough to prevent women with controlled hypertension or diabetes from taking HRT (Scientific Advisory Board, 1996).

\section{Contraindications}

The absolute contraindications to HRT include: history of unexplained vaginal bleeding, active liver disease, breast cancer, and active vascular thrombosis. In addition, cautions or specific monitoring should be given and low dosages are preferred in the conditions such as migraine, history of thromboembolism, familial hypertriglyceridemia, endometriosis, uterine cancer, gall bladder disease, and strong family history of breast cancer (Scientific Advisory Board, 1996). 


\section{Onset and Duration of Treatment}

The optimal time to start HRT and duration of HRT for preventing and treating osteoporosis are uncertain. Many studies suggest that all postmenopausal women should consider HRT/ERT (e.g., Whittington \& Faulds, 1994; Willhite, 1998; Cauley et al., 1995; Lindsay, 1987). Long-term treatment is required to achieve the full protective effect of HRT. The greatest benefit may occur if it is used for 10 years or more.

However, some studies argued that since there is a long-time interval between menopause and the peak incidence of fracture (from the seventh decade onwards), a five to ten yeartherapy initiated at menopause is unlikely to provide optimal protection against fracture some decades later (Adis International Limited, 1997). Although for women with menopausal symptoms, an/or at high risk of heart disease, initiation of HRT at menopause may be appropriate, starting HRT at menopause seems not cost-effective if short term intervention at a later stage can also prevent fractures.

Another issue is the rate of bone loss after cessation of therapy. Ettinger \& Grady (1993) stated that rapid bone loss will resume when HRT/ERT is discontinued, similar to that observed in untreated women at the onset of menopause. The protective effect of long term HRT is greatest at the end of treatment and will have decreased to approximately half after a 10-year period following treatment for 10 years. Thus, even 10 years of HRT cannot be expected to confer protection over the entire remaining life span. Several other studies also showed that although women who take estrogen have fewer fractures than those who do not, previous users of estrogen do not appear to have any significant residual reduction in fracture risk compared with women who never take 
estrogen after menopause (e.g., Ettinger et al., 1985; Felson et al., 1993; Kiel et al., 1987). Therefore, to significantly reduce fracture risk, HRT must be taken continuously. However, most postmenopausal use of estrogen is prescribed for treating menopausal symptoms. Once these symptoms are subsided, the majority of women discontinue estrogen use. The long-term compliance to HRT regimens is suboptimal and becomes a big concern (Meunier, 1999).

\section{Compliance}

Compliance to HRT is low, especially for a long-term use (Meunier, 1999; Salamone et al., 1996; Ravnikar, 1987). Therapy discontinuation and noncompliance are major problems for women taking HRT to prevent and treat osteoporosis. This is even true in symptomatic women (Whittington \& Faulds, 1994). Studies have indicated that of women who do receive a prescription for HRT, $30 \%$ never have it filled, $20 \%$ stop taking the medication within nine months (Ravnikar, 1987), and about one-fourth stop treatment within two years (Kushner, 1998). The major reasons for non-compliance include perceived lack of need for HRT and concern for adverse effects such as breast cancer and intermittent vaginal bleeding. The addition of cyclic progestin to an estrogen regimen will cause regular withdrawal bleeding in $50 \%$ to $80 \%$ of women, but the bleeding becomes less prevalent as the length of treatment increases.

The low treatment compliance and the risks/adverse effects associated with HRT make researchers and clinicians seek for more treatment solutions with high safety, efficacy, and effectiveness. Treatments other than HRT need to be considered, especially for patients contraindicated to HRT or not willing to take HRT for a long term. Other 
drug therapies such as alendronate and raloxifene also play an important role in osteoporosis prevention and treatment.

\section{Alendronate Therapy}

Alendronate was approved in November 1995 for the treatment of osteoporosis. It was approved in the summer of 1996 for the prevention of osteoporosis in postmenopausal women and recently, for steroid-induced osteoporosis. Dosage and Duration of the Treatment

Alendronate is prescribed at a continuous dose of $10 \mathrm{mg} /$ day for treatment and 5mg/day for prevention (Merck \& Co., Inc., 1995; Kushner, 1998). Currently, it is not clear on the optimal duration of alendronate therapy (Scientific Advisory Board, 1996).

\section{Effects on Bone Loss and Fractures}

Bisphosphonates are powerful inhibitors of osteoclastic bone resorption. Bisphosphonate drugs bind avidly to bone resorption sites, resist enzymatic degradation, and have half-lives similar to that of bone (Scientific Advisory Board, 1996). At present, etidronic acid, alendronic acid, and resedronic acid are approved by FDA for the treatment of osteoporosis. Etidronate inhibits bone mineralization at antiresorptive doses. Concerns regarding osteomalacia and increased fracture rates have diminished its role in the treatment of osteoporosis (Scientific Advisory Board, 1996). Residronate is very new and only few clinical studies have been reported so far.

Alendronate does not impair bone mineralization and it is the only FDA-approved bisphosphonate for the prevention and treatment of osteoporosis (Willhite, 1998). Many studies have shown that alendronate $10 \mathrm{mg}$ /day for two to three years effectively increases 
lumbar (5\% to $10 \%)$, remoral neck (1\% to $5 \%)$, trochanter $(7 \%)$, and distal forearm (0.3\% to $2.0 \%)$ BMD in osteoporotic women aged 44 to 84 years (Karpf et al., 1997; Chestnut et al., 1995). Alendronate 5mg/day for two years also produced significant increases in lumbar $(3.5 \%)$, femoral neck (1.9\%), and trochanter (3\%) BMD (Hosking et al., 1998), but not in distal forearm BMD. Unlike HRT, accelerated bone loss was not observed two years after discontinuation of alendronate. Therefore, the previous gains in BMD are maintained although bone loss resumes once a patient stops taking alendronate. The continued therapy is required to obtained progressive increase in BMD (Stock et al., 1997; Chestnut et al., 1995).

Furthermore, alendronate have been found to significantly decrease the risk of fractures, especially among high risk women. Data are available from several large, randomized, controlled clinical trials of up to 5 years duration in patients with osteoporosis or low BMD treated with alendronate (Liberman et al., 1995; Chesnut et al., 1995; Adami et al., 1995; Black et al., 1996; Bone et al., 1997; Karpf et al., 1997; Cummings et al., 1998). For example, a study conducted in post-menopausal women aged 51 to 88 years with at least one vertebral fracture at baseline showed that alendronae $10 \mathrm{mg} /$ day decreased new vertebral, hip, and wrist fractures by $47 \%, 51 \%$, and $48 \%$, respectively (Black et al., 1996). In another three-year controlled study of alendronate, the rate of new vertebral fractures was $48 \%$ lower in the treatment group than in the placebo group (Liberman et al., 1995). A meta-analysis of five placebocontrolled studies in osteoporotic women aged 42 to 85 without baseline fractures showed that alendronate reduced non-vertebral fractures by $29 \%$, hip fractures by $54 \%$, 
and forearm/wrist fractures by $61 \%$. Fracture reduction was seen in patients both younger and older than 65 (Karpf et al., 1997).

Efficacy and safety of bisphosphonates beyond 4 years are still unknown. The longest study duration so far is 4.25 year (Cummings et al., 1998). The research is continuing and more updated results will be expected.

\section{$\underline{\text { Other Benefits }}$}

There is no extra benefit of alendronate reported.

\section{$\underline{\text { Adverse Effects }}$}

Common adverse effects of alendronate therapy are gastrointestinal: dyspepsia, dysphagia, nausea, diarrhea, and constipation. The most serious side effects of alendronate is esophageal ulceration. However, apart from periodic monitoring of BMD to detect the occasional non-responder, more intrusive monitoring is usually unnecessary (Merck \& Co., Inc., 1995).

The adverse experience profile was similar for the patients treated with 5, 10, or $20 \mathrm{mg}$ doses of alendronate (Merck \& Co., Inc., 1995). The side effect rates reported in clinical trials and population-based studies or from actual clinical practice are diverse. In clinical trial studies, no significant difference was reported in the adverse experience rates between alendronate and placebo group (e.g., Merck \& Co., Inc., 1995; Black et al., 1996; Tucci et al., 1996). However, a frequency much greater than that reported in clinical trials was found by Ettinger et al. $\left(1998^{1}\right)$. About one in three women $(32.8 \%)$ taking alendronate for osteoporosis reported gastrointestinal symptoms. Furthermore, $12.3 \%$ of women taking alendronate for osteoporosis received healthcare for upper 
gastrointestinal disorder in a health plan population, of which $14 \%$ prompted hospital admission and $86 \%$ prompted outpatient visits. The risk of having GI side effects and a clinical encounter among alendronate users were 1.6 times higher than that among nonusers. This risk also increased with age and current use of NSAIDS.

Similarly, another study conducted by researchers at the Kaiser Permanente Medical Care Program of Northern California (KPNC) also found that alendronate use results in higher costs due to GI disorders (Levin, 1999). The direct health care costs of 792 women enrolled in KPNC, a large HMO, and took alendronate therapy were examined and compared to a population-based sample of the health plan's membership. The study showed that outpatient care was the major source of excess costs, which were \$40-\$67 per member per month higher than that of the control groups not using the medication. In addition, alendronate users were found to incur approximately $\$ 3.80$ more in monthly acid-related disorder inpatient expenses compared to the control groups. Excess pharmacy costs to treat acid-related disorders were also $\$ 2.80$ per member per month higher among the study group. Using the same database (KPNC), Ray et al. (2000) found that the clinic and pharmacy costs for acid-related disorders per patient were $\$ 715$, after adjustment for the age, gender, and comorbidities.

The difference in GI adverse event frequency may be due to differences in patient selection. Most clinical trials of alendronate excluded women who had active upper GI disease or who regularly use $\mathrm{H}_{2}$ blockers or NSAIDs (Liberman et al., 1995; Tucci et al., 1996; Black et al., 1996). In clinical practice, however, many women with osteoporosis also have GI diseases or use these drugs. Also, in clinical trials, participants are more 
likely to have received intensive counseling on the dosing instructions or medication adherence. The higher adverse event rate can furthermore cause a higher discontinuation rate of therapy in general practice than that in clinical trials.

Administration Requirements and Contraindications

Bisphosphonates, as a class, are poorly absorbed from the gastrointestinal tract and as a consequence, there are strict administration requirements. The drug need to be taken on an empty stomach, 2 or more hours before the next meal, with a full glass of plain water (not coffee, juice, tea, or mineral water). Furthermore, patients should remain fully upright and fasting for at least 30 minutes after dosing (Willhite, 1998). Failure to follow these requirement could result in a reduction in bioavailability and/or esphageal irritation or ulceration.

Alendronate is contraindicated in patients with esophageal abnormalities or inability to sit or stand upright for 30 minutes (Merck \& Co., Inc., 1995)

\section{Compliance}

Patients' compliance may be hampered by the strict administration requirements of alendronate. Patients' non-compliance is a serious issue due to gastrointestinal discomforts caused by inappropriate drug administration. Some clinical trial studies reported that the discontinuation rates of a 2-3 year therapy due to adverse reactions were $4.1 \%$ and $6.0 \%$ in the alendronate and placebo groups, respectively, for the dosage of $10 \mathrm{mg} /$ day, and $7.5 \%$ and $5.7 \%$ for the dosage of $5 \mathrm{mg} /$ day (Merck \& Co., Inc., 1995). However, Ettinger et al. $\left(1998^{1}\right)$ reported that the rate of discontinuation was linearly 
increasing over time and by the end of 10-month follow-up, almost half of the women (46.1\%) had discontinued alendronate treatment.

\section{Raloxifene Therapy}

Roloxifene is a selective estrogen receptor modulator (SERM). SERMs are "nonsteroidal tissue-selective modulators of estrogen receptor-mediated actions" (Willhite, 1998). As alternatives to HRT, SERMs positively affect some tissues such as bone and the cardiovascular system via estrogenic action, while lack estrogenic effects on the uterus. Therefore, SERMs are developed to optimize the positive ERT-like effects while decreasing or eliminating adverse events and risks of other diseases. So far, raloxifene is the only FDA-approved SERM for osteoporosis prevention in postmenopausal women.

\section{$\underline{\text { Dosage }}$}

The dosage of raloxifene for the prevention and treatment of osteoporosis and related fractures is $60 \mathrm{mg} / \mathrm{d}$ orally. There is no special requirement on the drug administration (Eli Lilly and Company, 1997).

\section{Effects on Bone Loss and Fractures}

Several randomized, double-blind, placebo-controlled, two-year studies have been conducted to examine the effects of raloxifene $(60 \mathrm{mg} / \mathrm{d})$ on BMD in postmenopausal women aged 40 to 60 years: the North American trial, the European trial, and the international trial (Eli Lilly and Company, 1997; Delmas et al., 1997). Results in all three studies showed that the mean total BMD percentage increased significantly at 12 and 24 months, compared with calcium-supplemented placebo groups. BMDs of the hip 
ranged from $1.3 \%$ to $2.4 \%$; the femoral neck, $1.6 \%$ to $2.5 \%$; the trochanter, $1.3 \%$ to $2.7 \%$; intertrochanteric, $1.3 \%$ to $2.3 \%$; and lumbar spine, $1.8 \%$ to $2.4 \%$. Conjugated estrogens produced increases in total hip BMD approximately twice that produced by raloxifene. Another study found that compared to the effects HRT and alendronate on BMD, raloxifene was slightly less effective at the hip and has approximately half effectiveness on lumbar vertebra (Delmas et al., 1997).

The effects of raloxifene on fracture risk are not thoroughly known yet. Research on the raloxifene in fracture risk reduction is ongoing. By now the Multiple Outcomes of Raloxifene Evaluation (MORE), a 3-year randomized clinical trial, is the longest trial for raloxifene (Ettinger et al., 1999). It was designed to examine the ability of raloxifene to treat postmenopausal women with osteoporosis and to prevent fractures in these women. Meanwhile, some secondary study end points such as the incidence of breast and endometrial cancer were also examined. More than 7,700 postmenopausal women participated in this clinical trial with a mean age of 66.5 years. Based on MORE study, reloxifene reduces the risk of first-time vertebral fracture in women with osteoporosis by $55 \%$ and reduces the risk of subsequent vertebral fracture by $30 \%$. Frequency of vertebral fracture was reduced both in women who did and did not have previous fracture. However, risk of nonvertebral fracture for raloxifene $v s$ placebo did not differ significantly, although raloxifene group had less fractures than placebo group.

\section{$\underline{\text { Other Benefits }}$}

Like estrogen, raloxifene has been proved to reduce major cardiovascular risk factors although it may be less effective than HRT (Walsh et al., 2000; Umland et al., 
1999; de Valk-de Roo et al., 1999). In a double-blind, randomized trial, 390 postmenopausal women aged 45 to 72 years used raloxifene $(60 \mathrm{mg} / \mathrm{d}$ and $120 \mathrm{mg} / \mathrm{d})$, HRT or placebo (Walsh et al., 1998). It found that raloxifene produced LDL decreases similar to those of HRT ( $12 \%$ and $14 \%$, respectively) and significantly lowered lipoprotein by $7 \%$ to $8 \%$ (HRT $19 \%$ decrease). Raloxifene increased $\mathrm{HDL}_{2}$, a subfraction of HDL that may be an important cardiovascular risk factor, by $15 \%$ to $17 \%$ versus $33 \%$ by HRT but did not significantly affect total HDL cholesterol. Triglycerides were unaffected and fibrinogen decreased by raloxifene. Another recent double blind, randomized, and placebo-controlled, 6-month clinical trial showed that raloxifene significantly lowered serum homocysteine levels which are independent risk factors for the development of cardiovascular disease by $8 \%$, and similar to the $7 \%$ reduction obtained with HRT (Walsh et al., 2000).

However, raloxifene's effects on cardiovascular end points such as morbidity and mortality of coronary heart disease have not been reported. More research need to be done to determine whether the beneficial effects produced by raloxifene on cardiovascular risk factors will translate into a reduction of cardiovascular risk.

Recently, with the release of interim data from the ongoing MORE study, there is a debate on the preventive effect of raloxifene on breast cancer. The MORE study showed that postmenopausal women with osteoporosis receiving raloxifene had a $70 \%$ reduced risk of developing newly diagnosed breast cancer, compared with placebo group (Ettinger et al., 1999). Similar results were produced by raloxifene 60 and 120mg/day group. Researchers in MORE study concluded that approximately two and a half years 
of treatment with raloxifene reduced the risk of breast cancer in postmenopausal women who had osteoporosis and no history of breast or endometrial cancer. However, the researchers also mentioned that since the study subjects were osteoporotic women and osteoporosis itself is associated with a reduced risk of developing breast cancer, caution must be given in interpreting the results. The MORE trial is continuing and the long-term effects of raloxifene on prevention of fractures and breast cancer are being studied. So far, there is no definite answer on whether raloxifene could prevent breast cancer or not. A study called STAR (Study of Tamoxifen and Raloxifene), which is comparing the efficacy of the two agents in the prevention of breast cancer in postmenopausal women, is on the way. More than 20,000 postmenopausal women will be recruited in this study. The results of this study are expected to provide a better explanation to the role of raloxifene in the prevention of breast cancer (Carlson, 1998).

The protective effects of raloxifene on bone, cardiovascular risk (through total and LDL-cholesterol reduction), and probably breast cancer risk make raloxifene as a promising agent with a "do-all" therapeutic profile for the postmenopausal women. Raloxifene may therefore offer better overall prevention for women than other agents such as HRT, alendronate and tamoxifen when the risks of endometrial cancer, fracture, and breast cancer are all taken into account. However, many researchers believe that it is too early to recommend this approach, as the clinical studies conducted to date have been designed to primarily evaluate effects on fracture risk and not breast cancer and cardiovascular disease prevention (Prestwood et al., 2000; Carlson, 1998).

\section{$\underline{\text { Adverse Events and Contraindications }}$}


Compared to HRT and alendronate, adverse effects of raloxifene is minimal. Raloxifene is well tolerated by most patients. Studies show that there is no significant difference in the incidence of adverse events between treatment group and placebo group (Eli Lilly and Company, 1997; Delmas et al., 1997). Compared to HRT, hot flashes are more common with raloxifene. Unlike estrogen, raloxifene does not relieve menopausal symptoms, and it may actually cause hot flashes. However, raloxifene appears to cause less breast pain, vaginal bleeding, flatulence, and abdominal pain (Eli Lilly and Company, 1997; Ettinger et al., 1999). Also, it does not stimulate the postmenopausal endometrium (Goldstein et al., 2000; Meunier et al., 1999).

Raloxifene's potential for causing deep venous thrombosis is similar to that of estrogen (Eli Lilly and Company, 1997; Kushner, 1998; Physicians’ Desk Reference, 1999). The MORE study showed that the risk of venous thromboembolus in women receiving raloxifene is three times higher than that in placebo group (Ettinger, 1999). It is rare but can cause serious consequences. Therefore, like HRT, Raloxifene is contraindicated in women with current or past thromboembolic events.

\section{Compliance}

Patients are expected to have relatively high compliance rate due to the good tolerance, easy administration and dosage form. Compared to HRT, Raloxifene treatment was reported to have less dropout rate (Willhite, 1998). A study conducted by Lilly Research Laboratories (1999) combined eight randomized clinical trials (6-30 months' duration) of raloxifene to examine the incidence of adverse events and dropout rate. It revealed that discontinuation rates were not significantly different between treatment 
groups and placebo group. Vaginal bleeding was responsible for significantly more discontinuations from the HRT groups compared with the raloxifene group. Hot flashes was significantly increased in the raloxifene group. However, it did not increase the discontinuation rates. Also, the incidence of leg cramps was greater in raloxifene group compared with placebo group, but did not cause any discontinuation of therapy. The study concluded that raloxifene can be well tolerated by women and the discontinuation rates were low compared to HRT.

\section{Pharmacoeconomic Research On The Three Drug Therapies}

Based on the clinical trial data, pharmacoeconomic research has been done to evaluate the cost-effectiveness, cost-utility of HRT, alendronate, and raloxifene in the treatment/prevention of osteoporosis. There are numerous pharmacoeconomic studies on HRT and most of them were published in 1980's and the early of 1990's . Pharmacoeconomic evaluations on alendronate and raloxifene are relatively rare, especially for raloxifene, due to inadequate clinical trial data. The following will review the multiple effects of the three drug therapies and the associated risk rates frequently used in the literature.

\section{Hormone Replacement Therapy (HRT)}

\section{Effects on Fracture Risk}

Many studies have been done in assessing the cost effectiveness of HRT for fracture prevention since 1980's. Early pharmacoeconomic studies usually based their analysis on a retrospective, case-control, 1-year study performed by Weiss et al. (1980), 
who found a relative risk (RR) of 0.43 for current users of estrogen (Weinstein \& Schiff, 1983; Cheung \& Wren, 1992; Daly et al., 1992). Various effectiveness rates (risk reduction rates) have been applied in the analysis. For example, Weinstein (1980) assumed a RR of 0.33 while patients received HRT, and 0.5 for a period after ceasing therapy equivalent to that of its duration. Tosteson et al. (1990) utilized a computer simulation model and used an estimated RR of 0.84 and 0.52 for women aged 50 to 74 years after 5 and 10 years of HRT, respectively. In general, a RR of 0.5 for all types of fractures has been used (Tosteson et al., 1990; Weinstein \& Tosteson, 1990).

Some pharmacoeconomic analyses examined fracture risk by fracture types. Hip and vertebral fractures are the two types of fractures most often studied. Researchers used a relative risk of hip fracture of 0.8 for the first 5 years of treatment and 0.4 thereafter, with the risk increasing gradually after stopping the treatment (Weinstein \& Schiff, 1983; Cheung \& Wren, 1992; Daly et al., 1992; Roche \& Vessey, 1990; Weinstein \& Tosteson, 1990). For example, in a cost-effectiveness analysis of HRT conducted by Cheung and Wren (1992), a hypothetical model of women of age 50 with and without HRT was established. The RR of hip fracture was assumed between 0.8 to 0.4 depending on treatment durations ( 5 - 15 years). A more recent study conducted by the Swedish hip fracture study group found that the risk of hip fracture decreased at a rate of $6 \%$ per year of HRT use with an RR of 0.36 (95\% CI, 0.24 to 0.53 ) for women currently taking HRT (Michaelsson et al., 1998). Rosner et al. (1998) compared cost-effectiveness of HRT, calcium, etidronate, and alendronate treatment in the prevention of vertebral fractures in 
postmenopausal women with osteoporosis. The risk of a vertebral fracture was assumed to decrease by $55 \%$ by HRT for 3 years ( $R R=0.45$, compared with no therapy).

Unlike other pharmacoeconomic studies, Clark and Schuttinga (1992) calculated health care cost savings of HRT in preventing osteoporotic fractures based on the fracture risk levels and possible usage of HRT (compliance). The study evaluated a hypothetical cohort of 100,000 American white women who reached 50 years of age during 1988. Baseline risks for hip fracture, vertebral fracture, and other fractures were estimated in high, medium, and low risk categories. Risk reduction rate of 50\% $(\mathrm{RR}=0.5)$ for total fractures after 5 years of HRT was applied for all three risk groups (i.e., the fracture incidence for each group was assumed to decrease by $50 \%$ with HRT). The study showed that the total net savings was $\$ 27.6$ million (1988 dollars) attributable to screening and HRT. Although this study did not state clearly how the three risk categories were classified (no classification criteria was reported), it is one the few studies that take into account the baseline risk levels and apply this to the cost-saving calculation.

Although treatment duration and types of fractures varied in pharmacoeconomic research, almost all studies concluded that long-term HRT for menopausal women, or at least for symptomatic women, is cost-effective. However, few studies have considered women's compliance with the treatment and examined how non-compliance affects the extent of cost-effectiveness of HRT. Effects on Cardiovascular Disease Risk 
Generally, a 50\% risk reduction rate was assumed for unopposed HRT after 10 years of treatment (Cummings et al., 1990² Cheung \& Wren 1992; Tosteson \& Weinstein, 1991). When progesterone is added to the estrogen replacement therapy, some pharmacoeconomic studies assume the effect on the relative risk of heart disease is the same as that of estrogen therapy (Whittington and Faulds, 1994). On the other hand, many pharmacoeconomic studies have assumed that the cardioprotective effect of HRT, compared that of ERT, is halved (Cheung \& Wren 1992; Daly et al., 1992; Roche \& Vessey, 1990) or completely negated (Tosteson \& Weinstein, 1991; Rosner et al., 1998). Some researcher argued that although long-term use of HRT could provide significant cardioprotective effects, there was also evidence that it could increase the risk of breast cancer (Rosner et al., 1998). The two effects may countervail to some extent. Also, these long-term effects may not be substantial due to the low patient compliance with HRT. Uncertainty in the effects of long-term HRT on heart cardiovascular disease and breast cancer as well as low treatment compliance rate make it disputable whether and how these extra benefits or risks should be included in pharmacoeconomic research.

\section{Effects of HRT on Breast Cancer}

As stated before, a definitive association between HRT use and breast cancer risk has not been established. Consequently, the relative risk rates used in pharmacoeconomic research are not consistent. Most of research did not include the increased risk of breast cancer, or only included it in a sensitivity analysis (e.g., Rosner et al., 1998; Weinstein, 1980). Daly et al. (1992) assumed that 5 years of HRT did not increase risk, but that after 10 and 15 years of treatment, the risks of breast cancer would increase by $30 \%(\mathrm{RR}=$ 
$1.3)$ and $50 \%(R R=1.5)$, respectively. The risk was assumed to remain elevated after treatment cessation for a period equal to that of treatment. Other studies applied relative risk rates of 1.25 (Weinstein \& Schiff, 1983; Weinstein \& Tosteson, 1990, Cheung \& Wren, 1992) or 1.36 (Tosteson \& Weinstein, 1991). The treatment costs of breast cancer were assumed to be $\$ 4,000$ in 1982 dollars (Weinstein \& Schiff, 1983).

$\underline{\text { Risk Rates Used for Other Benefits and Risks }}$

Cerebrovascular disease. In a study conducted by Daly et al. (1992), a cohort of postmenopausal women were followed with HRT for 10 years after onset of menopause at age 50 years. The relative risk of stroke with combined estrogen and progestrogen therapy was 0.88 to 1.0 , depending on duration of therapy. Roche and Vessey (1990) assumed that the effect of HRT on stroke was the same as that for heart disease: a $50 \%$ or $25 \%$ risk reduction after 10 years of ERT or HRT, respectively. The benefits of both therapies were assumed to be halved at 5 years duration of treatment, and to continue after HRT cessation for a period equal to the duration of treatment.

Gallbladder disease. Earlier pharmacoeconomic studies also examined the effect of HRT on the increased risk of gallbladder disease. A relative risk of 2.5 after 5 years of therapy was used (Weinstein, 1980; Weinstein \& Schiff, 1983). However, most pharmacoeconomic studies later did not include this effect in the analyses.

The use of HRT also increases the risk of vaginal bleeding, breast pain, venous thrombosis, and other side effects. Unscheduled uterine bleeding is the most common adverse events and may lead to a dilatation and curettage (Cheung \& Wren, 1992). The incidence of adverse events may decrease over time because of the discontinuation of the 
therapy, developed patients' tolerance, and/or adjusted dosages. Therefore, different adverse event rates were used for the first year and subsequent years of therapy in some studies (Rosner et al., 1998). Other studies also used a cumulative rate of the side effect for the treatment duration (Cheung \& Wren, 1992). Although most adverse events of HRT will not incur extra health care costs and are not considered by most pahramcoeconomic studies in their calculation of cost-effectiveness ratios, these side effects do decrease patients' quality of life and tolerance of the therapy. The impact of adverse events on the potential costs and effectiveness of HRT may be reflected by the reduced treatment compliance and utility (effectiveness). For example, in a costeffectiveness study conducted by Rosner (1998), only $21.5 \%$ of the patients were believed to be willing to continue HRT for 3 years based on the results from a previous study (Rosner et al., 1998; Ettinger et al., 1998²). Another study calculating health care cost-savings of HRT used a WTC (willingness-to-continue) rate of $90 \%$ for high-, $70 \%$ for mid-, and 0\% for low-risk group (Clark and Schuttinga, 1992). This study also found that the savings were sensitive to compliance, especially to the compliance of the highrisk group. Each increase of $10 \%$ in compliance resulted in increased net savings of $\$ 0.4$ million. The breakeven points of cost savings balance could be achieved with a compliance of approximately $70 \%$ and $40 \%$, for the high- and mid-risk groups, respectively. Therefore, it is very important to consider patients' compliance in pharmacoeconomic studies. Studies that ignore the patients' compliance or the impact of side effects on patients' quality of life may overestimate the cost-effectiveness of HRT. 


\section{Alendronate Therapy}

There are several large, prospective, randomized, controlled clinical trials that have provided valuable efficacy and safety data for alendronate in the prevention and treatment of osteoporosis and fractures (e.g., Cummings et al., 1998; Chesnut et al., 1995; Liberman et al., 1995). These trials have demonstrated approximately a 50\% reduction in vertebral, hip, and wrist fractures. The risk reduction rate was consistent across each of the studies and at each major site of osteoporotic fracture including the hip and wrist. For example, in the Clinical Fracture Study of the Fracture Intervention Trial (FIT), treatment with alendronate for a mean duration of 4.25 years was associated with a $50 \%$ and $56 \%$ reduction in the incidence of first vertebral fracture and hip fracture, respectively, among postmenopausal and osteoporotic women. (Cummings et al., 1998). In another study conducted by Liberman et al., treatment with alendronate overall (both $5 \mathrm{mg} /$ day and $10 \mathrm{mg} /$ day group) was associated with a $48 \%$ risk reduction of new vertebral fractures among postmenopausal and osteoporotic women, although the 5-mg dose was found less effective than the 10-mg dose.

On the other hand, only few pharmacoeconomic studies have been done that consider both the effectiveness and costs as well as compare alendronate with other alternatives (Rosner et al., 1998). Most clinical trial studies compared alendronate with “no therapy”, HRT, etidronate, or calcium, but no economic evaluation was conducted. However, alendronate is commonly believed as an effective drug in reducing the likelihood of fractures but at a cost that may be prohibitive to many patients (UllomMinnich, 1999; Truter \& Serfontein, 1999). 
Rosner (1998) compared four treatment strategies in the prevention of vertebral fractures in postmenopausal and osteoporotic women: two bisphosphonates (alendronate or intermittent cyclic etidronate plus calcium), HRT, and calcium therapy for three years. The baseline vertebral fracture risk (in "no therapy group") was assumed to be 390.3 vertebral fractures per 1,000 patient years. The relative risk of a vertebral fracture for alendronate compared with no therapy over 3 years was assumed to be 0.37 (risk reduction rate of $63 \%)$.

The major adverse event associated with alendronate use is upper gastrointestinal disorders. The incidence rates of GI adverse events reported in the literature are inconsistent. As mentioned before, the incidence rates widely ranged from nonsignificant to as high as $32.8 \%$. The cost-effectiveness study conducted by Rosner (1998) used a rate of 10.3 and 4.1 for minor GI for the first year and the subsequent second and third year of treatment, respectively. A rate of 1.5 and 0 for esophagitis/esophagael ulcer for the first year and the subsequent second and third year of treatment, respectively. However, they did not mention whether or how the treatment costs were included in the calculation of cost-effectiveness ratios. The compliance rate used in this study was estimated based on a post-marketing surveillance study of etidronate since no WTC values outside of clinical trials could be found for alendronate (Procter \& Gamble Pharmaceuticals, 1996). They assumed that only $24 \%$ of women were willing to continue their alendronate therapy for 3 years. 


\section{Raloxifene Therapy}

To date no pharmacoeconomic studies have been published that evaluate effectiveness and costs of raloxifene compared with its alternatives such as alendronate and HRT. Like alendronate, long-term effects of raloxifene are still unclear. The effects of raloxifene on other disease conditions such as breast cancer and heart disease are also unknown. This makes it difficult to evaluate the cost-effectiveness considering both short-term and long-term effects, and both benefits and risks on multiple organ systems. There is a great need of research to explore the clinical, economic, and humanistic effects of raloxifene in the prevention and treatment of osteoporosis.

\section{Challenges to Assessing Cost-Effectiveness of the Three Drug Therapies}

The accuracy and validity of pharmacoeconomic evaluations are markedly affected by the various assumptions made in the analyses on risks and benefits, compliance levels, and utility values. Many of the assumptions concerning risks and benefits made in the pharmacoeconomic studies have not been supported by current clinical data. For example, evidence is still lacking on the optimal duration of therapy, the change in relative risk after discontinuation of therapy, the risk reduction for cardiovascular/cerebrovascular diseases, and the risk increase for breast cancer and some long-term adverse events. Another challenge is how to estimate patients' compliance and utility values. Compliance levels are usually overestimated and utility values underestimated in pharmacoeconomic analyses (Whittington \& Faulds, 1994). Future pharmacoeconomic studies should estimate these values based on the most updated and valid studies instead of using arbitrary values. 
Controversial areas surrounding the assessment of treatment effectiveness also include the choice of appropriate health outcomes or end points for evaluation (i.e., BMD v.s. fracture averted v.s. quality-adjusted-life years gained), the skeletal sites that should be evaluated (i.e., vertebral v.s. non-vertebral fractures), and the duration of treatment effects. Many agents can maintain and may increase BMD level, but it is not clear whether such changes always relate to reduced fracture risk. Therefore, many researchers have proposed fractures as the preferred end point in both clinical trials and pharmacoeconomic studies. However, nonosteoporotic fractures have seldom been controlled for the evaluation. Another question is what types of fractures should be considered. Most published pharmacoeconomic studies are restricted to hip fractures. Vertebral fractures have rarely been included. Although hip fractures have deep impact on economics and patients' quality of life, risk reduction in all types of fractures risk should be considered for an accurate assessment of drug therapies.

The clinical and economic evaluation of the three preventive drug therapies is also related to the baseline fracture risk (i.e., population risk without any study treatment). The cost-effectiveness of an intervention varies among women at different risk levels (Seeman eta 1., 1995; Clark and Schuttinga, 1992; Kanis, 1984). For HRT and raloxifene that may provide benefits on multiple organ systems, the risk of diseases other than osteoporosis may also affect the effectiveness results. In most clinical and pharmacoeconomic studies, however, the subjects are women at the great risk for fracture (e.g., postmenopausal women with osteoporosis or with a previous fracture). Therefore, the efficacy and cost-effectiveness data of HRT, alendronate, or raloxifene obtained may 
compare favorably with other uses of health care resources only in selected high-risk groups. More pharmacoeconomic studies need to be done associating cost-effectiveness evaluations of preventive drug therapies with the study population risk levels. A valid risk assessment model is needed to evaluate the risk distribution for specific populations. Assumptions and baseline risk rates varied considerably in pharmacoeconomic evaluations. This makes it difficult to compare the results between studies. However, despite all of these challenges, literature-based pharmacoeconomic evaluation models are still useful in helping researchers and clinicians obtain a better understanding of the role of each competing drug therapy, and in developing health policy before clinical trials are completed. With the progress of the ongoing studies, the updated data can be used to validate and improve the existing evaluation models.

\section{Risk Prediction for Osteoporotic Fractures}

Prevention strategies are essential for reducing the incidence of osteoporosis and related fractures. Effective prevention requires identification of those at risk prior to fracture. Three common methods used to predict fracture risk independently or together are: (1) techniques to measure bone mass; (2) biochemical tests; (3) clinical risk factor analysis (Council of the National Osteoporosis Foundation, 1996). Although no single clinical finding or test result can quantify risk of postmenopausal osteoporosis and fractures with certainty, risk assessment can always help clinicians to identify women at increased risk. 
Bone mass (or BMD) and risk of osteoporotic fracture. Evidence of increased bone turnover from serum and urine markers and low bone mass as determined by densitometric techniques are highly predictive for osteoporosis and fracture risk. The greater the rate of bone loss or the lower the bone mass (especially the peak bone mass which usually achieved in the 20 s to 30 s of age), the greater the risk of osteoporosis and fracture (Scientific Advisory Board, 1996). Although osteoporosis may be heterogeneous, and although bone quality may also contribute to fracture susceptibility, BMD is usually used as an indicator of fracture risk. Both longitudinal and crosssectional studies have shown a strong relationship between low BMD and fracture occurrence (Ross, 1999; Ross et al., 1990). With each decline in bone mass of one standard deviation from the young adult mean fracture risk doubles. For example, osteopenia (BMD level between 1 and 2.5 SDs below the mean for young adults) carries a 2-fold increase in fracture risk compared with normal (BMD greater than 1 SD below the mean for young adult). And osteoporosis (BMD more than 2.5 SDs below the mean for young adults) carries a 4- to 5- fold increase in risk for fracture. The presence of previous osteoporotic fractures increases the risk of further fractures 20-fold (Scientific Advisory Board, 1996).

Other Risk factors of osteoporotic fracture. Risk factors other than BMD may also be used to estimate fracture risk. Age is one of the most important predictors in calculations of fracture probability. Risk factors such as Caucasian or Asian descent, slender body build, family history, smoking, alcohol abuse, low calcium diet, and sedentary lifestyle are also well documented. Some researchers suggest that frequency of 
falls and/or potential fall-related risk factors (e.g., physical capacity, neuromuscular function, mobility, visual function, and use of medications) should also be included as risk predictors for fractures (Lips, 1997; Ross, 1996; Dargent-Molina et al., 1996).

In general, the more risk factors a woman has, the higher the likelihood that she has or will develop osteoporosis/fracture. However, the effects of many risk factors have not been quantified, making it difficult to determine the importance of each risk factor and design most effective strategy to the target population.

In order to examine the association between various risk factors and the occurrence of osteoporotic fractures, estimate fracture risk in a population, and develop effective prevention strategies, a valid risk assessment model is needed. It will enable clinician to project an individual's lifetime fracture risk and therefore conduct preventive interventions. A few models have been developed to predict fracture risk through BMD and age (Ross et al., 1988' ; Carroll et al., 1997; Hui et al., 1988; Black et al., 1992; Kroger et al., 1995). Models that directly predict an individual's future risk of fractures from other risk factors mentioned above are scarce due to the difficulty of accurately predicting BMD level and fracture risk from risk factors for bone loss. BMD obtained from dual energy X-ray absorptiometry (DXA) scanning is so far the best predictor of osteoporosis and osteoporotic fracture. Measuring BMD directly is always recommended whenever possible (Ross, 1996).

On the other hand, measuring BMD to diagnose osteoporosis and predict future fracture may not be cost-effective for the whole population (mass screening), and in most situations, it is simply not applicable. Although the use of risk factors to predict BMD 
and fracture risk has been considered to be inadequate for precise diagnostic purpose, risk factors other than low BMD, may be helpful as a population-based screening tool to estimate the risk prevalence and determine who actually needs BMD assessment. There is a great need for establishing a population-based screening model using risk indicators for which data could be easily obtained.

Carroll et al. (1997) proposed a predictor equation for bone loss using BMD, age, years since menopause, and weight. The population to which this prediction model applied was normal and osteoporotic women aged 40 - 80 years. When a spinal BMD cutoff value of $0.86 \mathrm{gm} / \mathrm{cm}^{2}$ was used, their model had a sensitivity of $90 \%$ and a specificity of $60 \%$ for detecting women with vertebral fractures and was therefore defined as a high-risk BMD. Then they derived prediction curves using the prediction model they established to forecast the age at which that individual would reach the above-defined high-risk BMD. The person's expected number of remaining life-years at high risk for fractures thus could be estimated. This study provided a conceptual framework for constructing a risk assessment model for osteoporosis and related fractures through changes in bone loss rates. The prediction model they established had high sensitivity and fair specificity. However, the model only predicts high risk BMD, no direct connection between the risk factors (e.g., age, years since menopause, weight, family history, body mass, and calcium intake) and the clinical endpoint - fracture, was attempt to be made in this study.

Another study examined the predictive accuracy of objectively measured, selfreported, and recalled body mass index (BMI) for low BMD/osteoporosis (van der Voort 
et al., 2000). More than one thousand postmenopausal women aged 50-80 were asked for their present height and their weight at age 20-30 years. Subsequently their actual weight, height and BMD of the lumbar spine were measured. Predictive values (PV) and odds ratios were used to evaluate the prediction accuracies of the three factors. The study found the objective BMI test detected 50\% of the osteoporotic patients. And interestingly, the self-reported BMI test and the recalled BMI test had higher detection rates: $55 \%$ and $69 \%$, respectively. The study presents a possibility that detecting osteoporotic or low BMD patients by just measuring/asking a woman's weight and height. However, due to the low sensitivity of the prediction model, the authors suggested that age and BMI are not adequate when used for screening the population for osteoporosis.

Studies using risk factors to predict fractures are rare and the results are far away from satisfactory. Torgerson et al. (1996) conducted a prospective cohort study to build a fracture risk assessment model from BMD and other risk factors among perimenopausal women. Women aged 47-51 years were followed up for two years to assess the incidence of any self-reported fractures. The study revealed that the odds ratio (OR) for having at least one fracture during a 2-year perimenopausal period was 1.6 for every standard deviation $(\mathrm{SD})$ reduction in BMD at the spine. The fracture risk of women who were postmenopausal, or having a hysterectomy or prior history of fracture, was approximately twice than that of women without corresponding experience. A family history of fracture also significantly increased the fracture risk $(\mathrm{OR}=3.7)$. The researchers concluded that BMD measurements and other risk factors were predictive of perimenopausal fractures. 
Furthermore, they found that BMD at the hip and spine could also predict nonhip and nonspine perimenoausal fractures. However, no more information regarding model coefficients such as sensitivity and specificity was reported in their study. Another major limitation of this study is that the participating women were those who underwent a bone density measurement 2 years previously. This may cause a selection bias and greatly influence the interpretation of the results. The findings of this study may only applicable among high-risk women. Finally, the study was limited to a 2-year perimenoausal period, further follow-up is required to assess the predictive performance of BMD measurements and other risk factors for the long-term fracture risk.

Margolis et al. (2000) examined how body size influenced the risk of clinical fractures in older women (age $>=65$ years). Weight, weight change since 25 years of age, body mass index, lean body mass and percent body fat, and nonspine fractures were measured during an average 6.4 years of follow-up period. The study found the OR was from 2-2.4 for each factor when examined separately. Adjustment for total-hip BMD eliminated the elevated risk. Therefore, the study concluded that total body weight, as a factor can be easily measured, is useful in the risk assessment of clinical nonspine fractures (hip, pelvis, and rib fractures) when bone density measurements are not available. The results of this study also suggest that measurements of other attributes of body size or composition do not provide clinically relevant information about risk for hip, pelvis, and rib fracture beyond that provided by weight. Finally, measurement of body size or body composition contributes little to the clinical risk assessment for wrist, humerus, ankle, and foot fractures. This study did not control other risk factors such as 
calcium intake and family history. Also, spine fractures, a common type of fracture in women with osteoporosis were not examined.

Different techniques have been used to build risk assessment model. Multilogistic regression is the most commonly used tool to create the prediction model. Artificial Neural Network (ANN), a non-linear computational model has also been used in clinical diagnosis and classification. A study showed that the two techniques produce similar sensitivity, specificity, and accuracy in the prediction (Ongphiphadhanakul, 1997). Therefore, the unsatisfactory performance of the prediction models in the prediction of low BMD may be due to the lack of adequate association between BMD and the risk factors selected rather than the nature of the computational models.

In conclusion, osteoporosis may be detected before signs and symptoms occur through BMD tests and/or risk factor assessment. The challenge in the literature of this area is to obtain a risk function with high accuracy that predicts fracture risk based on major risk factors. Although there are a few such equations available (e.g., Tosteson et al., 1990; Melton 1988; Ross et al., 1988 ${ }^{1}$ ), questions remain about the validity of these models and applicability for different populations. 


\section{West Virginia Medicaid Program}

Medicaid is the joint federal and state funded health insurance program for the indigent, disabled, and members of families with dependent children (NPC, 1999). It is a federal-state partnership, with state laws and regulations, federally approved state waivers, and approved state plans. The federal government provides fiscal assistance and establishes regulations, guidelines, and policies that provide a framework within which the state governments operate their individual Medicaid programs. State governments are responsible for administration of the Medicaid program such as determination of eligibility and services provided, claims processing, and monitoring (NPC, 1999).

\section{Demographics of WV Medicaid Women Population}

Reflecting the gender, age, and race constitutions of the state, elderly white women compose a larger proportion of the total Medicaid eligibles population, compared to Medicaid programs in other states. The total number of female eligibles is 174,244 (58\%) in 2000. Among these women, more than $92 \%$ are white. Approximately $26 \%$ of white women are with age of 45 years or older. More specifically, the number of white female eligibles is 20,422 in the $45-64$ age group, 8,180 in the 65-74 age group, 7,526 in the 75-84 age group, and 5,858 in the 85+ age group (National Pharmaceutical Council, 2000).

\section{West Virginia Medicaid Spending Growth}

Medicaid programs nationwide have been plagued by escalating health care costs. Medicaid program costs have grown from $\$ 5.3$ billion in 1970 to $\$ 193.4$ billion by the end of 2000 , and is expected to grow to $\$ 335.7$ billion by 2007 . Although 
pharmaceuticals costs only account for a small proportion of total health care costs compared to hospital care, physician services, and long-term care services costs, it is the fastest growing component of the health care budget. Prescription drug costs in Medicaid programs have grown from $\$ 400$ million in 1970 to $\$ 19.7$ billion in 2000, and is expected to grow to $\$ 44.8$ billion by 2007 (Madhavan, 2000).

The national trend in healthcare costs is also evident in WV Medicaid program. Based on a recent review of WV Medicaid Program utilization and costs, the Medicaid drug expenditures per recipient increased dramatically, from less than $\$ 100$ in 1982 to over $\$ 700$ in 2000 (Madhavan, 2000). The percentage of drug expenditures in total Medicaid expenditures also increased from 7\% in 1982 to more than 15\% in 1999. The possible reasons for the significant growth in health care costs, especially pharmaceutical costs, have been widely discussed. For WV Medicaid program, a rapid growth in the number of recipients and introductions of new drugs with high price can contribute together to the increase in pharmaceutical costs. WV has high poverty rate and unemployment rate in its population. The Medicaid recipient population of prescription drugs had been doubled from 112,497 in 1982 to 274,214 in 1999 (Madhavan, 2000). Meanwhile, the R\&D costs had increased dramatically from $\$ 2$ billion in 1980 to about \$24 billion in 1999. New drugs are introduced at much higher prices that they were about several years ago.

The recent introduction of new chemo-preventive agents brings about a lot of concerns on the continuously increased drug budgets. Although most prevention interventions are more cost-effective comparing to the treatment, new drugs or 
technologies applied in the preventive treatment may add considerable present dollars and offset the possible benefits in the future. Under a cost-sensitive environment, it is extremely important for WV Medicaid policy maker to decide the most appropriate treatment compared to available alternatives, especially for preventive treatments, of which benefits can be only presented in the future with high uncertainty. 


\section{CHAPTER 3}

\section{METHODS}

The objectives of this study were: (1) to develop a decision model that compares the cost-effectiveness of three preventive drug therapies for osteoporotic fractures in postmenopausal women; (2) to assess the relationship between various risk factors and the occurrence of first osteoporotic fracture in the study population; (3) to determine the distribution of women on the basis of their probabilities for osteoporotic fractures in the study population; and (4) to examine the expected cost-effectiveness of the three drug therapies based on the fracture risk distribution in the study population. The study population consists of postmenopausal women who are enrolled in WV Medicaid and aged 45 to 85 years. To accomplish these study objectives, different methods were required. Hence, the study was completed in three phases.

\section{Phase I. Model Development}

Phase I constructed a decision model to identify and examine the relevant costs and consequences of HRT, alendronate, and raloxifene therapies for preventing osteoporotic fractures. The treatment costs and risk reduction rates of the three alternative drug interventions were identified. A hypothetical cohort of white women aged 45-85 years, postmenopausal, and without past incidence of osteoporotic fractures was studied in the model. The women in the cohort were treated with one of three treatment alternatives, and tracked over a period of 3 years. This length of intervention was chosen 
to coincide with the time period for which relevant clinical data from studies were available (Eli Lilly and Company, 1997; Carroll et al., 1997; Meunier et al., 1999; Rosner et al., 1998).

\section{$\underline{\text { Data Collection }}$}

Extensive literature review was conducted to obtain the relevant information. Studies that reported the direct relationship between the three drug therapies and the observed risk of fracture were identified by a MEDLINE search. Studies that reported the association between the drug therapies and BMD were not used, because BMD is an intermediary end point in terms of the primary outcome of interest - osteoporotic fractures.

Medicaid claims data (1999) were used to estimate the treatment costs. A Delphi panel of experts was also used to assess variables not available in literature or in the Medicaid database. The participating experts included a faculty in health services research from the School of Pharmacy, an internist, an endocrinologist, and a clinical pharmacist from West Virginia University Hospitals, and an internist from Johns Hopkins Hospital in Baltimore, MD. Experts' suggestions and opinions were collected in an iterative manners: first by an expert panel survey, then in a panel meeting, and finally by follow-up telephone calls. The expert panel questionnaire asked the experts about drug dosage forms and related patient distributions, drug adverse events' probability and recommended treatment, type of monitoring necessary for each therapy, major considerations for choosing each therapy, and other issues relevant to this study (Appendix C). 


\section{Model Structure}

Treatment effectiveness was measured in terms of the number of fractures averted by each drug therapy. Resource costs included the medication costs $\left(\mathrm{C}_{\text {Medication }}\right)$ of each treatment strategy, monitoring costs $\left(\mathrm{C}_{\mathrm{Monitoring}}\right)$, and the cost of the adverse effects $\left(\mathrm{C}_{\mathrm{AE}}\right)$. The expected economic savings associated with the additional benefits (other than preventing osteoporotic fractures) of each treatment, such as the risk reduction effects on cardiovascular disease, breast cancer, and hyperlipidemia, were not included in the model at this time since it is difficult to evaluate these effects from a short-term intervention and there are many uncertainties in this area. The cost-effectiveness ratios were thus defined as:

$$
\mathrm{C} / \mathrm{E}=\left(\mathrm{C}_{\text {Medication }}+\mathrm{C}_{\text {Monitoring }}+\mathrm{C}_{\mathrm{AE}}\right) /(\text { Number of Fractures Averted })
$$

The treatment duration was three years. No cessation effect was considered for all of the three therapies since a continuous, long-term therapy ( $>3$ years) was highly recommended in the prevention of osteoporotic fractures. Furthermore, the willingness of the woman to continue each therapy for three years (i.e., the probability of taking each drug for three years) was incorporated in the model. A cumulative discontinuation rate during the three-year study period was used. Figure 3-1 shows an example of the proposed decision tree. 


\section{Figure 3-1. The Structure of the Proposed Decision Tree.}

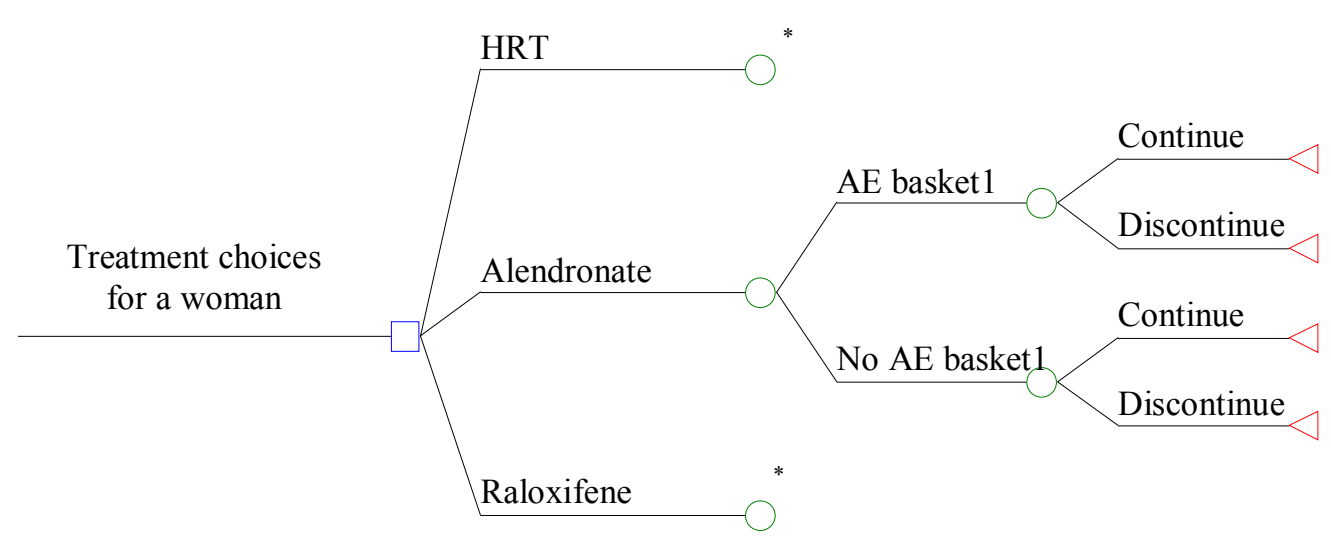

* The subsequent branches will be the same as those for alendronate.

Intervention Strategies and Effectiveness Parameter

The choice of three drug intervention strategies - HRT, alendronate, and raloxifene, was based on published guidelines and clinical practice. Doses, formulations, and patient distributions for the three drug therapies were determined to calculate appropriate treatment costs. The effectiveness variable was the number of osteoporotic fractures averted by each drug therapy. It was calculated as: $\left(\mathrm{I}_{0}-\mathrm{I}_{\mathrm{t}}\right)$ or $\left(\mathrm{I}_{0} * \mathrm{R}\right)$, in which $I_{0}$ was the fracture incidence (risk) of women not on any study treatments; $I_{t}$ was the fracture incidence (risk) of treatment group; $\mathrm{R}$ was the risk reduction rate by the treatment.

Based on the information available from clinical trial data, risk of any type of clinical fractures (more commonly hip and vertebra fractures, and less frequently wrist fractures) was considered. The two major fractures reported in literature are hip fracture 
which is the most costly and serious consequence of osteoporosis, and vertebral fracture which is the most common osteoporotic fracture. The clinical trial did not examine the effects of raloxifene on wrist fracture risk (Ettinger et al., 1999). The positive effect of alendronate on the risk of wrist is not definitive either. However, from the Medicaid point of view, the direct medical expenditures caused by wrist fracture is minimal. The effect of including wrist fractures had been shown to be negligible in previous studies (Johannesson \& Jönsson, 1993). For simplicity, risk reduction rates and treatment effectiveness (number of fracture averted) were not examined by different types of fractures. Nevertheless, the model developed in this study can be always applied to a specific type of fracture once the data on risk reduction rate for different types of fractures becomes available.

Fracture risk reduction rate associated with each therapy was obtained from published literature. When different published rates exist, the approximately median rate was chosen and subject to sensitivity analysis.

The incidence of fracture in the untreated women was viewed as the background risk and estimated from of West Virginia Medicaid women who do not have osteoporotic fracture experience, and have not taken any of the 3 preventive drug therapies. The risk distribution for the first osteoporotic fracture in this population is investigated in Phase II.

\section{Cost Parameters}

The direct costs of interventions included the average drug costs and monitoring costs for each drug therapy during the 3-year treatment. The perspective of cost analysis 
was that of third-party payers. All costs were calculated in 1999 prices (constant dollar method).

The average annual medication costs were estimated from the $1999 \mathrm{WV}$ Medicaid reimbursement rates. The brand name drugs were used for extracting average wholesale price (AWP) information from the database. For estrogen and progesterone, selected common brand name drugs were used to estimate the drug costs (Appendix A). The average of the highest and lowest prices was used as the medication AWP. The WV Medicaid medication reimbursement formula (AWP * 88\% $+\$ 3.90$ ) per prescription was then used to calculate the actual monthly and yearly Medicaid drug payments.

The cost of physician visits, endometrial monitoring (biopsy or aspiration curettage) for HRT and raloxifene and the cost of BMD tests for all three therapies were also added to the treatment costs. The number of follow-up physician visits was estimated based on literature (Johannesson \& Jönsson, 1993) and expert panel suggestion. Conservatively, three follow-up physician visits were needed per year for all three drug therapies. Also, based on the literature (Weinstein and Schiff, 1983; Johannesson \& Jönsson, 1993; Whittington \& Faulds, 1994) and experts' suggestion, we estimated that HRT would result in 1.5 extra mammography during the three-year study period compared to the "no therapy" group (increasing from biannual test to yearly test). In addition, 1.5 extra biopsy (a biannual examination) would be needed for HRT and raloxifene, and 2 extra BMD tests for all three therapies during the three-year study period. The CPT codes were used to extract the procedure cost based on $1999 \mathrm{WV}$ Medicaid reimbursement rate (Appendix B). 
Finally, the medical costs associated with adverse events of interventions were included, if there were any. Both clinical trial data and expert panel suggestions were used to identify these adverse effects and their probabilities as well as the related treatment procedures. Adverse events were grouped into "baskets" of symptoms based on underlying common body system affected, resource use, and cause. For example, the gastrointestinal basket included nausea, diarrhea, heartburn, etc. This will avoid doublecounting of physician visits that may occur for a group of symptoms rather than for each individual symptom. Only the side effects that could incur extra health care costs were examined in the study. The costs of OTC drugs that may have been used to treat side effects were also not included in the analysis. Since the study period is only three years, the long-term adverse effects were not considered.

The cumulative rates of the adverse events during the three-year treatment period were used to determine the probability of the woman experiencing each adverse event (AE). Then, the appropriate treatment cost was applied based on the treatment needed. According to the literature and experts' opinion, the major adverse events that need further treatment include unscheduled bleeding and DVT/leg pain for HRT, severe GI disorders for alendronate, and DVT/leg pain for raloxifene. Again, the CPT codes of the corresponding treatment procedures such as vaginal ultrasound, biopsy, and diagnostic ultrasound were used to obtain the unit cost information from 1999 WV Medicaid data (Appendix B). The costs for treating GI disorders were estimated from the literature (see Chapter 2: Ray et al., 2000; Levin, 1999; Ettinger, 1998). 
The monitoring costs and AE related treatment costs during the three-year treatment period were divided by three to obtain an average annual costs. Therefore, the total annual treatment costs would be the annual medication costs plus the annual monitoring and AE related costs. These total annual costs (assumed occurred at the end of each year) were then converted to the present value (the value same as that in the first year) using a discount rate of $3 \%$. The range of the discount rate tested in sensitivity analysis was from $2 \%$ to $5 \%$. The discounting formula was: NPV $=U(P / U, i, n)$, where $U$ is the annual costs, "( $\mathrm{P} / \mathrm{U}, \mathrm{i}, \mathrm{n})$ " is the discount uniform series factor, $\mathrm{i}$ is the discount rate, $\mathrm{n}$ is the number of years ( $\mathrm{Au} \& \mathrm{Au}, 1992)$.

\section{Treatment Discontinuation Rate}

Hormone Replacement Therapy. Based on the literature (see Chapter 2: Ettinger et al., 1998²; Clarkand Schuttinga, 1992; Rosner et al., 1998), a cumulative willingnessto-continue (WTC) rate of $25 \%$ for the 3 -year treatment was used in the model for HRT (i.e., an average annual discontinuation rate of $25 \%$ and a cumulative discontinuation rate of $75 \%$ were assumed). The range of cumulative WTC rate tested in the sensitivity analysis was from $20 \%$ to $30 \%$.

Alendronate Therapy. Information regarding patients' compliance with alendronate was inconsistent. The discontinuation rate of a 3-year therapy varied greatly from 7.5\% to 76\% (see Chapter 2: Merck \& Co., Inc., 1995; Rosner et al., 1998; Ettinger et al., $1998^{1}$ ). Since this study was a population-based study, data from general practice settings rather than clinical trials would be preferred. Therefore, a discontinuation rate of $60 \%$ or WTC rate of $40 \%$ at the end of 3 year was assumed (i.e., the modeled 
hypothetical woman would have $40 \%$ chance to complete the 3 -year treatment). Due to the great inconsistency in the literature, the range of $28 \%$ to $52 \%$ (base value $\pm 30 \%$ ) WTC rate was tested in the sensitivity analysis.

Raloxifene. Since there are no studies of treatment compliance with raloxifene in general practice, an assumption was made prudently based on the existing clinical trial data and recommendations in the literature (see Chapter2). There are many factors that may influence women's compliance with drug therapies such as side effects and treatment costs. Compared to HRT, raloxifene is unlikely to increase the risk of breast cancer, endometrial cancer, and vaginal bleeding. Thus, it seems safer for many women with a fear of these diseases and side effects. Other adverse events of raloxifene are similar to those of HRT. Although the extent to which how other factors such as drug costs affect compliance with raloxifene was not clear, we estimated that the discontinuation rate of raloxifene was much less than that of HRT, which is one of the most important advantages of raloxifene over HRT reported in research and practice. The hypothetical woman in the model will be assumed to have a relatively high WTC rate of $70 \%$. Conservatively, the average annual discontinuation rate of raloxifene was $10 \%$. Due to the lack of knowledge about patients' compliance with raloxifene, the range of cumulative WTC rate tested in the sensitivity analysis was from $49 \%$ to $91 \%$ (base value $\pm 30 \%)$.

How to model the cost and effectiveness for the non-compliant group is always a question. Few research studies have incorporated treatment compliance into a decision tree model and made the assumptions clearly regarding the possible reduced costs and 
effectiveness. For preventing osteoporosis and related fractures, long-term treatment is necessary. It has been suggested that all three drug therapies should be continually used for several years to demonstrate their positive effects on fracture risk. In this short-term study, both treatment effectiveness (risk reduction rate) and costs were assumed to occur in a linear way over the 3-year study period (Rosner et al., 1998).

\section{Main Assumptions}

The key assumptions in the construction of this model are:

- Because of the difficulty in differentiating osteoporotic fractures from fractures due to other causes, we assumed that women over 45 years of age and having a vertebral, wrist, or hip fracture, had osteoporosis and their fractures were osteoporotic fractures.

- Fracture risk reduction rates were obtained from published literature. When different rates were reported, the median value was chosen given the three-year study period and subject to sensitivity analysis over its reported range.

- Probabilities of adverse events were the average incidence rate for all major adverse event baskets. The effects of minor or rare adverse events were assumed negligible for all three therapies. A woman with adverse events but no additional treatment costs needed (e.g., breast tenderness, or minor GI with only over the counter drugs needed) would be included in the "No AE" group. For HRT, since the chance of having DVT is very small $(<1 \%)$, having both DVT and vaginal bleeding was assumed to be a very rare situation and not considered in this model. Also, for both HRT and raloxifene treatment, less than $1 \%$ of women may have 
diagnostic ultrasound due to the leg pain and if the ultrasound results are positive, further anticoagulant treatment (heparin + warfarin) may be needed. The costs of anticoagulant therapy are minimal due to the small probability. Therefore, only the ultrasound costs were included and the further anticoagulant treatment costs were ignored.

- The discontinuation rates were assumed to be same for women with and without adverse events since the overall cumulative rates for the 3-year treatment period were used.

- No study has been done in modeling the decreased effects of the three therapies after the termination of treatment (Jönsson, 1998). In this study, the treatment effects (risk reduction rate) and costs were assumed to occur in a linear way over the 3-year period. The average treatment discontinuation time was assumed to occur at the end of each year. The effect of the medications was assumed to begin immediately on start of therapy and to cease immediately on discontinuation (i.e., no delayed effects). Therefore, the treatment costs for the dropout women can be calculated based on the average annual treatment costs and the retention rates by the end of each year. The treatment effects (risk reduction rates) for the dropout women were assumed to be approximately $50 \%$ of the full effects (the average of the best effects and the least effects).

- In practice, women who use each drug therapy may also use vitamin D supplements and/or take calcium. The overall influence of medications other than 
HRT, alendronate, and raloxifene on health outcomes are assumed to be same for all three interventions.

- Treatment and prevention of osteoporosis induced by chronic use of high-dose corticosteroids are not examined in this study.

- The effectiveness of each therapy is not discounted in this study. Discounting health benefits through long-term preventive measures is controversial, because of the potential to excessively devalue downstream benefits (Fuchs \& Zeckhauser, 1987; Lipscomb et al., 1996; Jillman \& Kim, 1995). Whereas some researchers suggest that the same discount rate should be applied to both healthcare costs and health benefits, others argue that health benefits should not be discounted. The costs are discounted at $3 \%$ per year. This relatively low discount rate (comparing to $5 \%$ which is commonly used in the literature) is used because long-term treatment with three preventive drugs are desired and the low discount rate can avoid underestimating long-term benefits (Fuchs \& Zeckhauser, 1987; Lipscomb et al., 1996; Jillman \& Kim, 1995). Although in this model, only 3-year treatment duration is used due to the limited clinical trial information, the model is expected to incorporate long-term treatment costs and effects whenever it is possible.

\section{Phase II. Analysis of Background Risk}

In the hypothetical model built in Phase $\mathrm{I}$, one key variable - $\mathrm{I}_{0}$, which stands for the fracture risk in the women without any treatments, needs to be defined to calculate the number of fractures averted. The results of cost-effectiveness analysis are affected by the 
background fracture risk which varies in different populations. To apply the decision analytic model and calculate cost-effectiveness ratio to the study population, data are needed on the disease risk without the interventions. A risk assessment study of osteoporotic fractures needs to be conducted in the study population. The objective of Phase II is therefore to build a prediction model and estimate the distribution of women with different background risks for osteoporotic fractures in the study population. The prediction model is based on those widely reported risk factors such as age, family history, and body mass index (see chapter 2).

\section{Sample Selection}

A retrospective, case-control study design was used in Phase II of the study. The WV Medicaid data for 1998 and 1999 were used to extract information for women who were aged $45-85$ years and had claims with a date of service between January 1, 1998 to December 31, 1999. The number of cases in women between 45 and 64 years of age were expected to be small in the WV Medicaid data. Also, diagnosis information was not available for all women over 65 years of age in the Medicaid population since Medicare is the primary source of coverage for those 65 years or older. Therefore, two approaches were utilized for the selection of cases and controls for each age group (Figure 3-2). The approximate number of woman recipients in the two age groups was close: 21,000 in 4564 age group v.s. 19,000 in 65-85 age group in 1999 (WV Medicaid Internal Report, as of 9/17/1999). Thus, the sample size of cases and controls was the same for the two age groups. 


\section{Figure 3-2. Data extraction for the two age groups.}
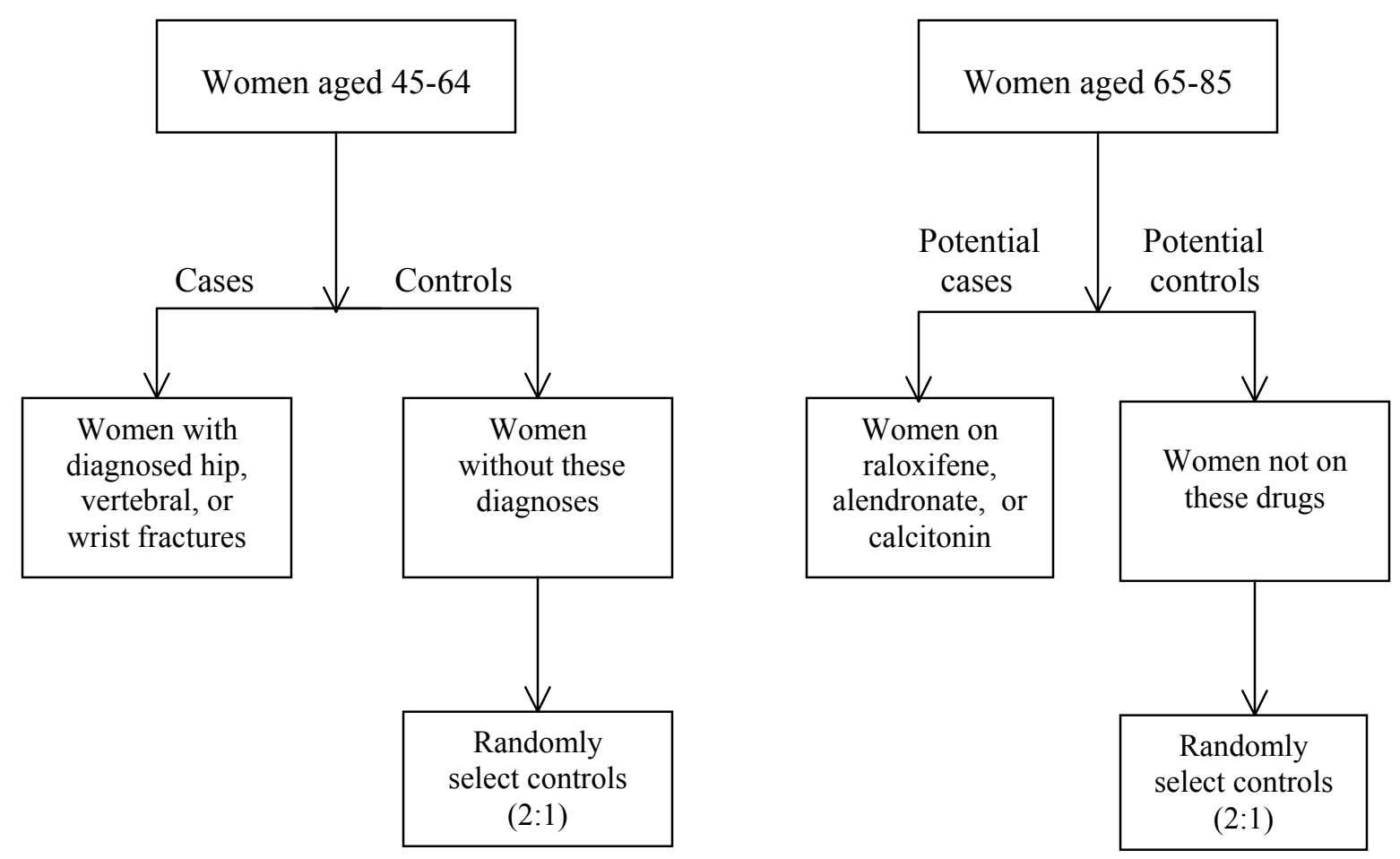
Sample selection among women aged 45 to 64: Cases were all women with diagnosed hip, vertebral, and/or wrist fractures between January 1, 1998 to December 31, 1999, a two-year period deemed sufficient to generate adequate number of cases in this younger age group. ICD-9 codes of the relevant diagnoses were used to identify and select case samples (Appendix D). The sample size goal was approximately 500 for cases. Once the cases had been identified, controls would be randomly selected from the remaining 45 to 64 age group using a 2:1 ratio of controls to cases. The sample size goal was approximately 1,000 for women who would serve as controls.

Sample selection among women aged 65 to 85: The potential cases were identified on the basis of prescription drugs used for treating osteoporotic fractures such as raloxifene, alendronate, and calcitonin. Potential controls for this older group were women randomly selected from those who were not on any of the above medications.

Note, the eventual number of cases and controls for both age groups might change later based on self-reported information from the survey. Women who reported never having a fracture in the case groups and women who reported having fracture experience after 45 year-old in the control groups would be excluded from the analysis.

Inclusion and Exclusion Criteria: Generally, women were eligible for this study only if they were aged 45 to 85 years and experienced menopause. Also, there were some specific selection criteria for cases and controls. For cases, the screening question was "Have you ever had an osteoporotic fracture?" Women with an answer of "No" would be excluded. For control group, there were two screening questions. One was "Have you ever had an osteoporotic fracture?" Women with an answer of "Yes" would be excluded 
from the control group. The second screening question asked the women whether they had ever taken any of the three drug therapies for bone weakness or for menopausal symptoms. Women who checked any one of the three treatments would be excluded from the control group.

\section{Sample Size Estimation}

A multiple logistic regression model was used to establish the equation model for fracture risk assessment. Under the assumptions that $\alpha$ is 0.05 (i.e., the probability of type I error is $5 \%$ ), $\beta$ is 0.2 (i.e., $80 \%$ detection power), an average probability of exposure in controls is $10 \%$, odds ratio of exposures between cases and controls is 2 , and the ratio of control to case is 2 , the estimated case sample size would be 205 for uncorrected chi-squared test and 223 for Fisher's exact test or corrected chi-squared tests (Strom 2000; Schlesselman 1982; Casagrande et al., 1978; Fleiss 1981).

Based on the response rates of previous studies in the WV Medicaid population (Ambegaonkar et al., 1997; Al-Momani et al., 1999), the response rate for this study was expected to be about $20 \%-30 \%$. Assuming a response rate of $20 \%$, the 3,000 surveys would generate approximately 600 responses (200 cases and 400 controls). Therefore, the sample size of 600 , if achieved, would be adequate to run the analysis for the prediction model.

\section{$\underline{\text { Data Collection }}$}

Data were collected over a two-month period from October to November 2000. The IRB approved final surveys were accompanied by a cover letter from the Medicaid Commissioner and mailed to all cases and controls (Appendix E). A second mailing of 
the questionnaire took place after 3 weeks to non-respondents of the first mailing. A sample of non-respondents to both mailings (cases: 50; controls: 100) was sent an abbreviated survey to determine critical demographic and study variables and reasons for nonparticipation to assess non-response bias.

\section{Instrument Construction}

A self-administered questionnaire was mailed to the selected sample. The final versions of the survey and cover letters are presented in Appendix E. The questionnaire has three parts: (1) Demographics, (2) Health Profile, and (3) Risk Assessment. The first two parts asked women about their demographic characteristics (e.g., age and education level), general health, and co-morbidity. As already mentioned, screening questions with regarding the osteoporotic fracture and menopause status were also included. In addition, the use of medications that may affect bones were assessed by asking women whether they had been taking steroids, thyroid medications, diuretics, sedatives, and antiseizure drugs. The commonly used brand names of these medications were listed to help women answer this question.

The Risk Assessment part measures several well-established risk factors for osteoporosis and osteoporotic fractures among the sample women. These factors included: a) age, b) age at menopause, c) current body mass index (BMI), d) peak BMI, e) family history, f) calcium and vitamin D intake, g) smoking, h) alcohol consumption, i) exercise; and j) physical job. Since more than $96 \%$ of WV population including the Medicaid population are white, race was not included in the model. 
The variables of age, age at menopause, current weight and height (which are used to calculate current BMI), and peak weight and height (which are used to calculate peak BMI) were examined by an open question directly asking the value of these variables. Calcium intake was evaluated by asking women about their daily consumption of some selected dairy products based on calcium supplements tables (Willhite, 1998; Sagraves et al., 1993; Calcium Supplements, 1989). Smoking and alcohol consumption were investigated by two questions with frequency-categorized responses. Finally, to simplify the questionnaire and reduce the respondents' burden, family history, vitamin D intake, exercise status, and whether women had a physical job were evaluated by "Yes"/"No" questions.

While the first two parts of Demographics and Health Profile were common for both cases and controls, the risk assessment part was different for the two groups. Women in the control group were surveyed mainly about their current risk factor information. Women in the case group and reporting fractures were asked to report their present age and age at the time of the first osteoporotic fracture. These women were also asked about their risk behaviors prior to the first osteoporotic fracture. Several answers were listed to help women to recall, and respondents were instructed to check all that apply.

\section{$\underline{\text { Data Analysis }}$}

Statistical Package for Social Sciences (SPSS-version 8.0) was used for all data analyses. Multiple logistic regression analysis was used to investigate the relationship between various risk factors and the occurrence of fracture and to establish a prediction 
model. The dependent variable was thus the fracture status, and the independent variables were the risk factors discussed above. Women's health status and affecting medication use were also included in the model to control their effects since these variables may confound the study relationship. The odds ratio (OR) was calculated for each predictor. Important model parameters such as goodness of fit, sensitivity, specificity, positive predictive value (PPV), negative predictive value (NPV), and overall percentage accuracy classification (PAC) were determined.

The $c$ statistic, a frequently used measure of the ability of a model to discriminate between the two groups of cases was also examined. The $c$ statistic can be interpreted as the proportion of pairs of cases with different observed outcomes in which the model results in a higher probability for the cases with the event than for the case without the event (SPSS Inc., 1999). The $c$ statistic ranges in value from 0.5 to 1 . A value of 0.5 means that the model is no better than assigning cases to groups by chance. A value of 1 means that the model always assigns higher probabilities to cases with the event than to cases without the event (SPSS Inc., 1999). The $c$ statistic is equal to the area under the Receiver Operating Characteristic (ROC). Therefore, ROC analyses was conducted to obtain the $c$ statistic.

To validate the model generated in logistic regression, the respondent data would be split into two subsets by randomly selecting $50 \%$ of subjects. One-half of the subjects (selected subjects) would be used in model building, and the other half (unselected subjects) would be used for the model validation. Statistics and classification results 
were generated and compared for both selected and unselected samples. This provided a mechanism for partitioning the data to perform a sound validation test (Wright, 1995).

Furthermore, the validated model was applied to all the controls to estimate the fracture probability. The predicted fracture probability for each individual in the control group calculated based on the model was saved as a new variable. Then the risk distribution (mean, standard deviation, range) can be estimated. Since the control women were randomly selected sample from the population, the fracture probability distribution in this sample can be inferred to the fracture-free women in the study population.

More specifically, the logistic model yielded a constant term $\left(b_{0}\right)$ and regression coefficients for the significant predictor variable $\left(b_{1,2,3, \ldots}\right)$. Then the predicted probability (P) of fracture occurrence in the control group can be calculated by the following steps (Wright, 1995):

$$
\mathrm{g}=\mathrm{b}_{0}+\mathrm{b}_{1}\left(\mathrm{x}_{1}\right)+\mathrm{b}_{2}\left(\mathrm{x}_{2}\right)+\mathrm{b}_{3}\left(\mathrm{x}_{3}\right) \ldots
$$

Then,

$$
\mathrm{P}=\mathrm{e}^{\mathrm{g}} /\left(1+\mathrm{e}^{\mathrm{g}}\right)
$$

For example, if the regression analysis shows $\mathrm{b}_{0}=10, \mathrm{~b}_{1}=.8, \mathrm{~b}_{2}=-.5, \mathrm{~b}_{3}=-.1$, and $\mathrm{x}_{1}$, $\mathrm{x}_{2}, \mathrm{x}_{3}$ represent age, weight, exercise (yes/no), respectively, for a 65 -year-old, 110-pound woman with no exercise habit, her fracture probability will be:

$$
\begin{aligned}
& \mathrm{g}=10+.8 * 65-.5 * 110-2 *(0)=7 \\
& \mathrm{P}=\mathrm{e}^{7} /\left(1+\mathrm{e}^{7}\right)=1.95 / 2.95=.66
\end{aligned}
$$

So, this woman will have a probability of $66 \%$ for developing a fracture. 


\section{Data Base Management}

The Medicaid data was only accessible to the principal investigator. After data cleaning and extraction, the recipients were assigned an identification number and all traceable personal identifiers (e.g., SSN and name) were removed from the claims data prior to analysis. Completed anonymous surveys were maintained by the principal investigator and only aggregate data were used in research reports. The principal investigator takes all responsibility for obtaining, organizing, analyzing, and maintaining the data base used in this study.

\section{Phase III. Cost-Effectiveness Analysis}

Phase III combined the information obtained from Phase I and Phase II to evaluate the cost-effectiveness of the three preventive drug therapies based on different levels of osteoporotic fracture risk in the study population. The economic impact of each drug therapy was examined for the whole WV Medicaid study population, and suggestions on the coverage policy were made to the WV Medicaid program.

\section{Conventional Cost-Effectiveness Analysis Using Mean Values}

A decision-analytic model incorporated all the relevant information from Phase I and Phase II. The cost-effectiveness (CE) ratio defined by the formula of $\mathrm{C} / \mathrm{E}=$ $\left(\mathrm{C}_{\text {Medication }}+\mathrm{C}_{\text {Monitoring }}+\mathrm{C}_{\mathrm{AE}}\right) /($ Number of Fractures Averted) was calculated for each therapy using a decision tree construction. The software DATA ${ }^{\mathrm{TM}}$ (version 3.5 for Healthcare, TreeAge Software Inc.) was used to construct the decision tree and conduct cost-effectiveness analysis as well as sensitivity analyses. A preliminary analysis used the 
"Roll Back" method to calculate CE ratios. The mean or base value of the direct medical costs and risk reduction rate obtained from Phase I, and the mean risk of a woman without any interventions estimated from Phase II will be used in the "Roll Back"

procedure (actually, in $\mathrm{DATA}^{\mathrm{TM}}$, during all analyses other than Monte Carlo simulation, the value taken from a distribution will always be its mean).

Simple sensitivity analyses in which variables were tested individually were utilized to evaluate key assumptions and test the robustness of the model. The stability of the results was assessed by varying the assumptions for each critical study variable. The highest and lowest possible values for each cost, effectiveness, probability, and compliance variable were tested in the model. Those values were decided based on the literature, expert panel survey, or assumptions made by the researchers. Through simple sensitivity analysis, variables that had significant impact on the results would be identified.

\section{Probabilistic Analysis and Monte Carlo Simulation}

Probabilistic analysis considers all possible cases/situations simultaneously. It allows researchers to assign plausible ranges and/or estimated distributions to the input variables. In other words, for each variable used in the formula, a range of values and their probabilities of occurrence are used as input instead of a single value. Since inputs are probabilistic, most of uncertainty or risk inherent in the intervention is reflected in the range of input variables. A computer program using a method called Monte Carlo simulation can be run to generate hundreds of variations of input variables' combinations (i.e., hundreds of scenarios) and corresponding output values for an intervention or 
strategy.

In this study, a probabilistic analysis (Monte Carlo simulation) was conducted to calculate the probabilistic CE ratio for each thearpy based on the distributions of background fracture risk $\left(f\left(\mathrm{I}_{0}\right)\right)$, treatment costs $(f(\mathrm{C}))$, and fracture risk reduction $(f(\mathrm{R}))$ in the treatment group. Then, the expected values (median costs per fracture averted per woman) were estimated for the three preventive drug therapies among fracture-free women in the study population. For a better understanding of the value of the three therapies, incremental effects and costs relative to the next most effective strategy were also calculated for each treatment strategy.

Specifically, the formula used as the base of Monte Carlo simulations was:

$$
f[\text { costs } /(\text { fracture averted })]=f(\mathrm{C}) /\left[\left(f\left(\mathrm{I}_{0}\right) \times f(\mathrm{R})\right]\right.
$$

in which $f(\mathrm{C})$ was the distribution of treatment costs for the treatment, $f\left(\mathrm{I}_{0}\right)$ was the distribution of background fracture risks, and $f(\mathrm{R})$ was the distribution of fracture risk reduction rates. In this study, the $f\left(\mathrm{I}_{0}\right)$ was estimated from the actual risk distribution in the study population obtained from Phase II. The distributions of treatment costs and fracture reduction rates were estimated by Triangle distribution using high, basic, and low values. Unlike using a fixed mean value in the conventional CE analysis for the treatment costs, risk reduction rate, and the fracture risk in the non-study group, the probabilistic $\mathrm{CE}$ calculation took into account the distributions of these variables simultaneously.

In order to evaluate how cost-effectiveness of each drug therapy was affected by the fracture risks in the study population and illustrate the potential economic and clinical 
outcomes, a matrix of expected CE ratios by risk levels (e.g., $<10 \%, 10 \% \leq$ Risk $<20 \%$, etc.) and by preventive drug strategies were created. It would then be possible to compare cost-effectiveness of various combinations of the three therapies based on the fracture risk levels. The recommendations to the WV Medicaid program on the coverage policy of the preventive drug therapies were made based on the comparison results.

The advantages of probabilistic $\mathrm{CE}$ analysis over the conventional $\mathrm{CE}$ analysis are obvious. Information obtained from a probabilistic simulation is superior to that obtained from a single-value sensitivity analysis (Torries, 1997). It takes into account the distributions of input values rather than just uses a single mean value or a value that occurs the most frequently. Its ability to focus on unknowns in data elements can reduce major concerns caused by these uncertainties for most decision makers.

\section{Limitations of the Study}

The study has several limitations inherent in the data. Those include:

1. The main limitation of this study is that the data currently available makes it difficult to simulate the cost-effectiveness values precisely. For example, the effects of raloxifene and HRT on risks and benefits of multi-organ systems are not clear. However, even minor changes in the relative risks of these conditions may have profound effect on cost-effectiveness ratios. If HRT proves beneficial in the prevention of heart disease, the issue about osteoporosis prevention may become unimportant. Although it is impossible for this study to incorporate the most accurate 
information in the economic assessment model, it should be possible to improve the accuracy and precision of the analysis as new data become available.

2. When evaluating the outcomes of chronic diseases and treatments, a lifetime horizon is favored. The model is designed to consider only three-year or less effects of each therapy and hence ignored long-term effects. Currently, the long-term effects of alendronate and raloxifene are still unknown. The long-term use of HRT may provide significant cardioprotective effects but there was also evidence that it could increase the risk of breast cancer. Additionally, because of the low patient compliance with HRT, whether these long-term effects would result in a substantial difference is unknown. Therefore, the long-term effects of all three drug therapies are difficult to measure and incorporated into the study model at present.

3. Patients' compliance is an important issue, especially for HRT and alendronate therapy. The relationship of treatment duration and treatment effects is unknown. Future research is needed to evaluate the discontinuation effects for each studied drug therapy.

4. In the risk assessment survey, all disadvantages associated with mail survey, especially recall bias, will impair the accuracy of the inferences made about the population. However, we feel that these effects will not significantly alter the results because the questions were made as simple and clear as possible and several possible answers were listed to improve the accuracy of responses. In addition, questions with too many missing values were reevaluated and were not included in the analysis. 
Finally, the same background risk distribution was used for all three therapies. Therefore, the recall bias may not affect the cost-effectiveness conclusion.

5. More information is required to further validate the risk prediction model and enhance its application. There is a great need for well-designed longitudinal studies to examine the association between bone density, clinical risk factors, and risk of fractures.

6. Inconsistency may exist within the literature on treatments' costs and effectiveness. But the probabilistic analysis used in this study can minimize discrepancies and identify most likely values.

7. In the literature, although there are a few fracture prediction models developed by clinical and population-based studies, no recognized cutoff values for categorizing risk levels, and no fracture incidence rates for each risk group have been reported. The model developed in this study is based on the WV Medicaid population, the generalization of study results (e.g., cutoffs for risk classification and CE ratios at each risk level) to other populations may not be appropriate.

8. A major disadvantage associated with probabilistic analysis is that many decision makers do not understand probabilistic evaluation and how to interpret the results. The result distribution itself does not indicate which intervention should be chosen. Multiple factors such as the decision makers' attitude toward the risk and the probability and amount of maximum costs for preventing one fracture should be considered together to make a decision (Torries, 1997). Although the analysis provides greater amount of useful information in a more accurate way, decision 
makers may feel more comfortable with a single value, even it is less precise or even misleading. However, as the discipline of pharmacoeconomics expands to include more disease and prevention/treatment modeling, probabilistic analysis and Monte Carlo simulations are expected to become more commonly used and will be gradually familiarized and accepted by decision makers.

9. This study assessed the cost-effectiveness of the three study drugs for the population. However, the most appropriate therapy for an individual woman may vary from the recommendation about the population.

10. Some may argue that regardless of fracture risk and treatment cost-effectiveness, drug therapies should always be considered as long as there is a probability of fracture, albeit low. Decisions about care are always difficult, especially in case of osteoporotic fractures with high morbidity and mortality rates. Only descriptive analyses and suggestions will be made in this study. Decisions on which therapy is preferred and who should obtain the drug therapies will depend upon individual decision makers and their special circumstances. 


\section{CHAPTER 4}

\section{RESULTS}

\section{PHASE I. MODEL DEVELOPMENT}

Phase I built an economic evaluation model for HRT, alendronate, and raloxifene therapies in the prevention of osteoporotic fractures. A hypothetical white woman aged 45 years or older and without past incidence of osteoporotic fractures was treated with one of the three alternatives: HRT, alendronate, or raloxifene, and was tracked for 3 years. To calculate the cost-effectiveness ratios, the corresponding costs and risk reduction rates need to be evaluated.

\section{Decision Tree Structure and Relevant Variables}

The software DATA ${ }^{\mathrm{TM}}$ (version 3.5 for Healthcare, TreeAge Software Inc.) was used to construct the decision tree and conduct the cost-effectiveness analysis. Figure 4-1 shows the structure and relevant variables of the decision tree. 
Figure 4-1. The Structure of Decision Tree*

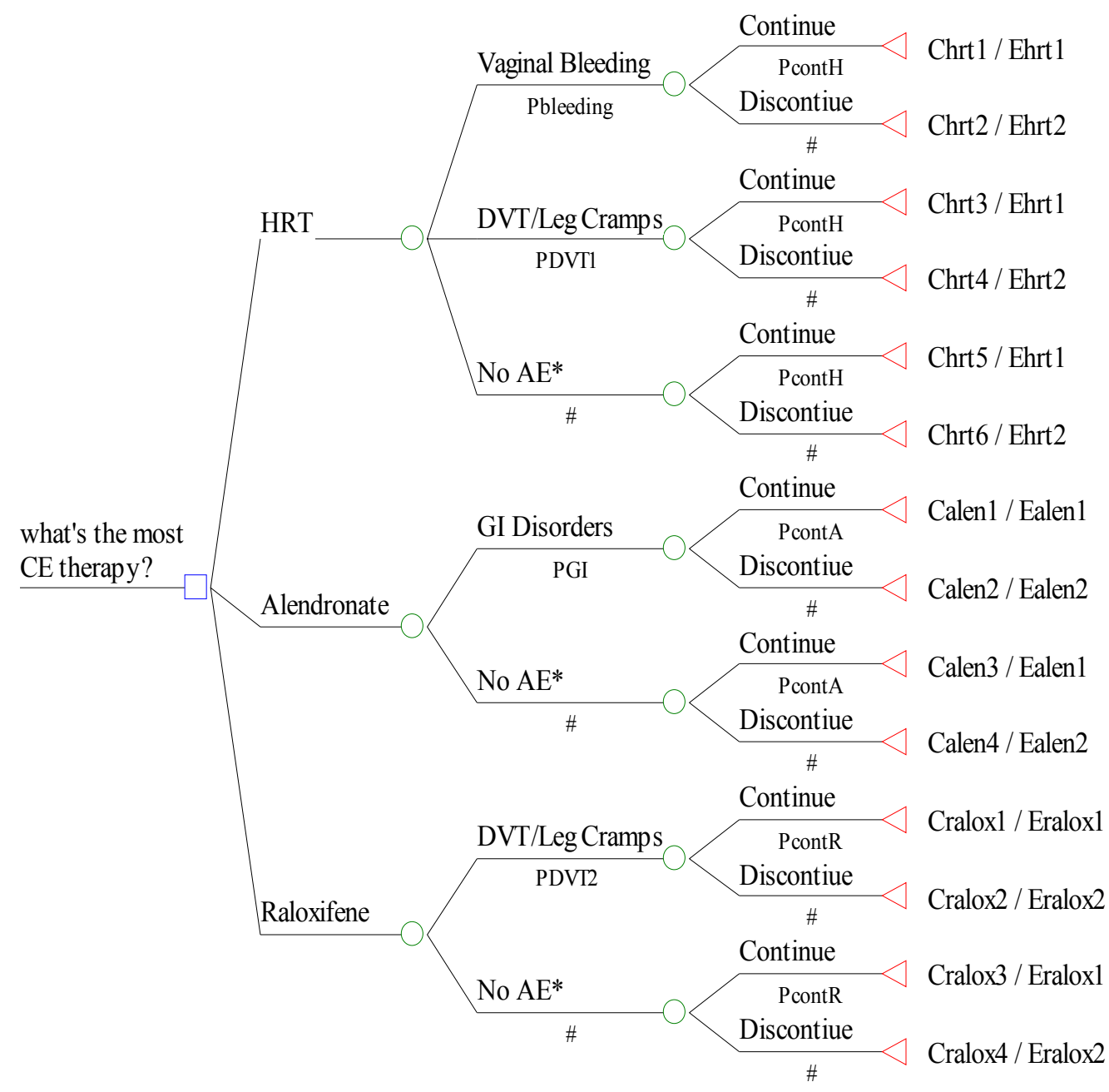

${ }^{*}$ The variables shown in the decision tree (Pbleeding, Calen1, Eralox1 etc.) will be described below in detail. 


\section{Intervention Strategies}

Doses, formulations, and patient distributions for the three drug therapies included in the model were based on clinical guidelines and Delphi panel suggestions (Table 4-1). Results from expert panel survey revealed that for HRT, most women (90\%) were on oral cyclic or continuous schedules. Only $10 \%$ of women were on an expensive trandermal schedule. This estimation was consistent with the literature (Rosner et al., 1998).

\section{Cost Parameters}

The direct cost of interventions included the average costs of drugs, monitoring costs, and adverse event costs for each drug therapy during the 3-year treatment. All costs were calculated in 1999 dollars.

Medication costs: The total costs paid by Medicaid per prescription (for one month) was calculated as: [(Unit AWP price $) *(1-0.12) *($ Usage/month $)+\$ 3.90]$. Therefore, the annual costs were calculated by multiplying monthly costs by 12 (Table 42). Ten representative products were used to calculate the medication costs: Premarin ${ }^{\circledR}$, Ortho-Est $^{\circledR}$, Ogen $^{\circledR}$, Menest $^{\circledR}$, Estratab $^{\circledR}$, Provera $^{\circledR}(5 \mathrm{mg}$ and $2.5 \mathrm{mg})$, Climara $^{\circledR}$, Fosamax $^{\circledR}$, and Evista ${ }^{\circledR}$ (Appendix A). The weighted average of the medication costs/person/year for HRT, alendronate, and raloxifene were \$338.92, \$664.56, and $\$ 689.88$, respectively. 
Table 4-1. Therapy Formulations and Patient Distribution for Each Dosage Form Based on Clinical Guidelines and Delphi Panel Estimates

\begin{tabular}{|c|c|c|}
\hline Therapy & Therapy Dose and Formulation & $\begin{array}{l}\text { Patient } \\
\text { Distribution (\%) }\end{array}$ \\
\hline \multirow[t]{4}{*}{ HRT } & $\begin{array}{l}\text { Cyclic estrogen } 0.625 \mathrm{mg} / \text { day }+ \text { cyclic } \\
\text { Medroxyprogesterone } 5 \mathrm{mg} / \text { day }\end{array}$ & $45 \%$ \\
\hline & $\begin{array}{l}\text { Continuous estrogen } 0.625 \mathrm{mg} / \text { day }+ \\
\text { continuous Medroxyprogesterone } 2.5 \mathrm{mg} / \text { day }\end{array}$ & $45 \%$ \\
\hline & $\begin{array}{l}\text { Cyclic transdermal estradiol } 50 \mu \mathrm{g} / \text { day }+ \text { cyclic } \\
\text { Medroxyprogesterone } 5 \mathrm{mg} / \text { day }\end{array}$ & $5 \%$ \\
\hline & $\begin{array}{l}\text { Continuous transdermal estradiol } 50 \mu \mathrm{g} / \mathrm{day}+ \\
\text { continuous Medroxyprogesterone } 2.5 \mathrm{mg} / \text { day }\end{array}$ & $5 \%$ \\
\hline Alendronate & $5 \mathrm{mg} /$ day & $100 \%$ \\
\hline Raloxifene & $60 \mathrm{mg} /$ day & $100 \%$ \\
\hline
\end{tabular}




\section{Table 4-2. Medication Costs (1999 dollars)}

\begin{tabular}{lccc}
\hline \multicolumn{1}{c}{ Treatment } & $\begin{array}{c}\text { Actual } \\
\text { (i) }\end{array}$ & $\begin{array}{c}\text { Annual } \\
\text { Paid Costs/Rx } \\
\text { (ii) }\end{array}$ & $\begin{array}{c}\text { Costs } \\
\text { (iii) }\end{array}$ \\
\hline HRT & 21.77 & 23.06 & 276.72 \\
$\quad$ Cyclic combined schedule (pill) & 34.84 & 34.56 & 414.72 \\
$\quad \begin{array}{l}\text { Cyclic combined schedule (patch) } \\
\text { Continuous combined schedule (pill) }\end{array}$ & 31.20 & 31.36 & 376.32 \\
$\quad$ Continuous combined schedule (patch) & 41.62 & 40.53 & 486.36 \\
Adjusted by usage ${ }^{\dagger}$ & - & 28.24 & 338.92 \\
Alendronate & 58.50 & 55.38 & 664.56 \\
Raloxifene & 60.90 & 57.49 & 689.88 \\
\end{tabular}

1. $($ ii $)=($ i $) * .88+3.90$;

2. $\quad($ iii $)=($ ii) $* 12$;

3. $\dagger$ calculated based on the patient distribution as showed in Table 4-1. 
Monitoring costs: Besides the medication costs, the costs of physician visits, endometrial monitoring (biopsy or aspiration curettage) for HRT and raloxifene, and the cost of BMD tests for all three therapies were added to the treatment costs (Table 4-3). According to the literature (Weinstein and Schiff, 1983; Johannesson \& Jönsson, 1993; Whittington \& Faulds, 1994) and experts' suggestion, HRT would results in 1.5 extra mammography at the cost of $\$ 11 /$ test during the 3-year study period compared to the "no therapy" group (increasing from biannual test to yearly test). Also, 1.5 extra biopsy (a biannual examination) would be needed for HRT and raloxifene. The costs were approximately $\$ 106 /$ biopsy. Furthermore, the expert panel suggested that the BMD test should be conducted for all the three therapies every 1 to 2 years. Therefore, approximately 2 extra BMD tests were needed during the 3-year treatment for each therapy, at a cost of $\$ 39 / \mathrm{BMD}$ test. Finally, three follow-up physician visits were needed per year for all three drug therapies, at a cost of $\$ 24 /$ visit, compared to no therapy group. Thus, the average monitoring costs during the 3-year treatment period was $\$ 469.50$ (\$156.50/year) for HRT, \$294 (\$98/year) for alendronate, and \$453 (\$151/year) for raloxifene (Table 4-3). 
Table 4-3. Average Monitoring Costs During the 3-year Treatment Period

\begin{tabular}{|c|c|c|c|c|c|c|}
\hline \multirow[b]{2}{*}{ Treatment } & \multicolumn{2}{|c|}{ HRT } & \multicolumn{2}{|c|}{ Alendronate } & \multicolumn{2}{|c|}{ Raloxifene } \\
\hline & Utilization & Costs $(\$)$ & Utilization & Costs $(\$)$ & Utilization & Costs $(\$)$ \\
\hline Physician visits & 9 & 216 & 9 & 216 & 9 & 216 \\
\hline Mammography & 1.5 & 16.5 & 0 & 0 & 0 & 0 \\
\hline Endometrial biopsy & 1.5 & 159 & 0 & 0 & 1.5 & 159 \\
\hline BMD test & 2 & 78 & 2 & 78 & 2 & 78 \\
\hline Total monitoring costs & - & 469.5 & - & 294 & - & 453 \\
\hline
\end{tabular}


Costs related to adverse events: Both clinical trial data and expert panel suggestions were used to identify these adverse effects and their incidence rates as well as the related treatment/diagnostic procedures. The cumulative rates of the adverse events during the three-year treatment period were used to determine the probability of the woman experiencing each adverse event. The major adverse events that need additional treatment and incurred costs are listed in Table 4-4. All costs were examined based on 1999 prices and reported in 1999 dollars.

The major adverse events that may require extra medical care include unscheduled bleeding and DVT/leg pain for HRT, severe GI disorders for alendronate, and DVT/leg pain for raloxifene. The extra costs for treating GI disorders were estimated from the literature (see Chapter 2: Levin, 1999; Ettinger, 1998). The GI disorders included were peptic ulcer, gastric ulcer, gastroesophageal reflux disease, gastritis, esophagitis, dyspepsia, nausea, vomiting, and abdominal pain (Ettinger, 1998). The average outpatient care costs (clinic and pharmacy costs) were approximately $\$ 700$ per person per episode (Ray et al., 2000, Levin, 1999; Ettinger, 1998). Other reimbursement rates were obtained from 1999 WV Medicaid database based on CPT codes (Appendix B). The extra AE costs for HRT and raloxifene during the 3-year treatment period were $\$ 197$ (\$65.67/year) and \$47 (\$15.67/year), respectively (Table 4-4). 
Table 4-4. Adverse Events and Related Treatment Costs

\begin{tabular}{|c|c|c|}
\hline $\begin{array}{l}\text { Therapy } \\
\& \text { AE }\end{array}$ & $\begin{array}{l}\text { Treatment } \\
\text { needed }\end{array}$ & Related Costs (\$) \\
\hline \multicolumn{3}{|l|}{ HRT } \\
\hline $\begin{array}{l}\text { Unscheduled } \\
\text { bleeding }\end{array}$ & $\begin{array}{l}\text { Vaginal ultrasound and } \\
\text { endometrial biopsy }\end{array}$ & $\begin{array}{c}44 \\
106\end{array}$ \\
\hline DVT/Leg pain & Diagnostic ultrasound & 47 \\
\hline $\begin{array}{l}\text { Alendronate } \\
\text { GI disorders }\end{array}$ & $\begin{array}{l}\text { Outpatient care } \\
\text { (clinic visit \& Rx drugs) }\end{array}$ & 700 \\
\hline \multicolumn{3}{|l|}{ Raloxifene } \\
\hline DVT/Leg pain & Diagnostic ultrasound & 47 \\
\hline
\end{tabular}


The monitoring costs and AE related treatment costs during the three-year treatment period were divided by three to obtain an average annual costs. Therefore, the total annual treatment costs with or without AE can be calculated. These total annual costs were assumed to occur at the end of each year and were converted to the present value (the value same as that in the first year) using a discount rate of $3 \%$. The range of the discount rate tested in sensitivity analysis would be from $2 \%$ to $5 \%$. The discounting formula was: $\sum \mathrm{PV}=\sum\left[\mathrm{U}^{*}(\mathrm{P} / \mathrm{U}, \mathrm{i}, \mathrm{n})\right]$, where $\mathrm{U}$ is the annual costs, $(\mathrm{P} / \mathrm{U}, \mathrm{i}, \mathrm{n})$ is the discount uniform series factor, $\mathrm{i}$ is the discount rate, $\mathrm{n}$ is the number of years ( 1 to 3 ) (Au $\& \mathrm{Au}, 1992)$. At this time, the treatment costs were calculated for the entire 3-year treatment period (i.e., the woman completed the treatment). The results are presented in Table 4-5. 


\section{Table 4-5. Complete Treatment Costs Summary}

\begin{tabular}{ccc}
\hline & $\begin{array}{c}\text { Average Annual Costs } \\
\text { (Constant \$) }\end{array}$ & $\begin{array}{c}\text { Total Costs } \\
\text { (Present Value } \$)\end{array}$ \\
\hline HRT & & \\
$\mathrm{C}_{\text {drug }}+\mathrm{C}_{\text {monitoring }}$ & & $1,401.35$ \\
$\mathrm{C}_{\text {drug }}+\mathrm{C}_{\text {monitoring }}+\mathrm{C}_{\text {bleeding }}$ & 545.42 & $1,542.78$ \\
$\mathrm{C}_{\text {drug }}+\mathrm{C}_{\text {monitoring }}+\mathrm{C}_{\mathrm{DVT}}$ & 511.09 & 1445.67 \\
Alendronate & & \\
$\mathrm{C}_{\text {drug }}+\mathrm{C}_{\text {monitoring }}$ & & $2,156.99$ \\
$\mathrm{C}_{\text {drug }}+\mathrm{C}_{\text {monitoring }}+\mathrm{C}_{\mathrm{GI}}$ & 762.56 & $2,816.99$ \\
Raloxifene $_{\mathrm{C}_{\text {drug }}+\mathrm{C}_{\text {monitoring }}}$ & 995.89 & \\
$\mathrm{C}_{\text {drug }}+\mathrm{C}_{\text {monitoring }}+\mathrm{C}_{\mathrm{DVT}}$ & & $2,378.52$ \\
& 840.88 & $2,422.85$ \\
\hline
\end{tabular}

* All costs were calculated in 1999 dollars.

${ }^{* *}$ A discount rate of $3 \%$ was used with a range of $2 \%-5 \%$ tested in the sensitivity analysis. 


\section{Treatment Discontinuation}

In reality, not all women can complete their treatment. In another word, there is a probability that the studied hypothetical woman could discontinue her treatment during the 3-year period. To calculate the costs correctly, treatment continuation rate or willingness-to-continue (WTC) rate should be considered. Table 4-6 presents the cumulative WTC rate for the three-year treatment and WTC rate at the end of each year. According to the literature, the dropout rate among HRT users is high. The cumulative probability or percentage of discontinuing the treatment was $75 \%$. That means the hypothetical woman in this study had only $25 \%$ chance to complete the 3 -year treatment. The average annual discontinuation rate was $25 \%$ for HRT. For women who took alendronate, about $40 \%$ women remained after 3-year treatment. The average annual discontinuation rate was $20 \%$. Women on raloxifene had a relatively high WTC rate. The cumulative WTC rate was $70 \%$ and the average annual dropout rate was $10 \%$. The treatment costs of the discontinuation group was assumed to occur linearly over the 3-year period. They were calculated based on the WTC rate by the end of each year. The same method used in the calculation of total treatment costs (present value) for the completion group was utilized for the discontinuation group (Table 4-7). 
Results

Table 4-6. Treatment Willingness-To-Continue (WTC) Rate (\%)

\begin{tabular}{lcccc}
\hline & $\begin{array}{c}\text { Cumulative } \\
\text { WTC Rate }\end{array}$ & Year 1 & Year 2 & Year 3 \\
\hline HRT & 25 & 75 & 50 & 25 \\
Alendronate & 40 & 80 & 60 & 40 \\
Raloxifene & 70 & 90 & 80 & 70 \\
\hline
\end{tabular}




\section{Table 4-7. Treatment Costs Summary for Discontinuation Group}

\begin{tabular}{|c|c|c|c|c|}
\hline & \multicolumn{3}{|c|}{ Average Annual Costs (Constant \$) } & \multirow{2}{*}{$\begin{array}{c}\text { Total Costs } \\
\text { (Present Value) }\end{array}$} \\
\hline & Year 1 & Year 2 & Year 3 & \\
\hline \multicolumn{5}{|l|}{ HRT } \\
\hline $\mathrm{C}_{\text {drug }}+\mathrm{C}_{\text {monitoring }}$ & 371.57 & 247.71 & 123.86 & 707.59 \\
\hline $\mathrm{C}_{\text {drug }}+\mathrm{C}_{\text {monitoring }}+\mathrm{C}_{\text {bleeding }}$ & 409.07 & 272.71 & 136.36 & 779.00 \\
\hline $\mathrm{C}_{\text {drug }}+\mathrm{C}_{\text {monitoring }}+\mathrm{C}_{\mathrm{DVT}}$ & 383.32 & 255.55 & 127.77 & 729.96 \\
\hline \multicolumn{5}{|l|}{ Alendronate } \\
\hline $\mathrm{C}_{\text {drug }}+\mathrm{C}_{\text {monitoring }}$ & 610.05 & 457.54 & 305.02 & $1,302.69$ \\
\hline $\mathrm{C}_{\text {drug }}+\mathrm{C}_{\text {monitoring }}+\mathrm{C}_{\mathrm{GI}}$ & 796.71 & 597.53 & 398.36 & $1,701.29$ \\
\hline \multicolumn{5}{|l|}{ Raloxifene } \\
\hline $\mathrm{C}_{\text {drug }}+\mathrm{C}_{\text {monitoring }}$ & 756.79 & 672.70 & 588.62 & $1,907.50$ \\
\hline $\mathrm{C}_{\text {drug }}+\mathrm{C}_{\text {monitoring }}+\mathrm{C}_{\mathrm{DVT}}$ & 770.90 & 685.24 & 599.59 & $1,943.06$ \\
\hline
\end{tabular}

${ }^{*}$ The costs were assumed to occur in a linear way over the 3-year period.

${ }^{* *}$ A discount rate of $3 \%$ was used. 


\section{Effectiveness Variables}

The effectiveness variable was the number of osteoporotic fractures averted by each drug therapy. It was calculated as: $\mathrm{I}_{0} * \mathrm{R}$, in which $\mathrm{I}_{0}$ was the fracture incidence rate of "no therapy group"; and R was the risk reduction rate by the treatment. The risk reduction rates and the range tested in sensitivity analysis are presented in Table 4-8. The estimation was based on literature reports: both previous pharmacoeconomic studies and the most updated clinical trials (see Chapter 2). The rates were adjusted for the shorter study duration compared to most clinical trials. Therefore, the rates were lower than those used in some pharmacoeconomic studies. For women who completed the 3-year treatment, the risk reduction rates of any clinical fractures were $40 \%$ for HRT, $50 \%$ for alendronate, and $55 \%$ for raloxifene therapy. The average risk reduction rates used for women who stop the therapy were $20 \%, 25 \%$, and $27.5 \%$ for HRT, alendronate, and raloxifene, respectively.

In summary, the treatment costs, risk reduction rates, and probability variables listed in the decision tree (Figure 4-1) are described in Table 4-9. 
Table 4-8. Risk Reduction Rates Used for the Three Preventive Drug Therapies (for a 3-year treatment).

\begin{tabular}{ccc}
\hline Therapy & $\begin{array}{c}\text { Risk Reduction Rate } \\
\text { (any clinical fractures) }\end{array}$ & Range Tested \\
HRT & $40 \%$ & $20 \%-60 \%$ \\
Alendronate & $50 \%$ & $45 \%-65 \%$ \\
Raloxifene & $55 \%$ & $30 \%-60 \%$ \\
\hline
\end{tabular}


Results

Table 4-9. The Value of Costs and Probability Variables

\begin{tabular}{|c|c|c|c|}
\hline Variables & Description & Base Value & Range Tested \\
\hline Costs $(\$)$ & & & $\pm 10 \%$ \\
\hline Chrt1 & costs of HRT: have bleeding, continue & $1,542.78$ & \\
\hline Chrt2 & costs of HRT: have bleeding, discontinue & 779 & \\
\hline Chrt3 & costs of HRT: have leg cramps, continue & $1,445.67$ & \\
\hline Chrt4 & costs of HRT: have leg cramps, discontinue & 729.96 & \\
\hline Chrt5 & costs of HRT: no AE, continue & $1,401.35$ & \\
\hline Chrt6 & costs of HRT: no AE, discontinue & 707.59 & \\
\hline Calen1 & costs of alendronate, have GI, continue & $2,816.99$ & \\
\hline Calen2 & costs of alendronate, have GI, discontinue & $1,701.29$ & \\
\hline Calen 3 & costs of alendronate, no AE, continue & $2,156.99$ & \\
\hline Calen4 & costs of alendronate, no AE, discontinue & $1,302.69$ & \\
\hline Cralox 1 & costs of raloxifene, have leg cramps, continue & $2,422.85$ & \\
\hline Cralox 2 & costs of raloxifene, have leg cramps, discontinue & $1,943.06$ & \\
\hline Cralox 3 & costs of raloxifene, no $\mathrm{AE}$, continue & $2,378.52$ & \\
\hline Cralox 4 & costs of raloxifene, no $\mathrm{AE}$, discontinue & $1,907.50$ & \\
\hline \multicolumn{4}{|l|}{ Effectiveness } \\
\hline Ehrt1 & risk reduction rate by HRT, continue & 0.40 & $0.20-0.60$ \\
\hline Ehrt2 & risk reduction rate by $\mathrm{HRT}$, discontinue & 0.20 & $0.10-0.30$ \\
\hline Ealen1 & risk reduction rate by alendronate, continue & 0.50 & $0.45-0.65$ \\
\hline Ealen2 & risk reduction rate by alendronate, discontinue & 0.25 & $0.225-0.325$ \\
\hline Eralox1 & risk reduction rate by raloxifene, continue & 0.55 & $0.30-0.60$ \\
\hline Eralox2 & risk reduction rate by raloxifene, discontinue & 0.275 & $0.15-0.30$ \\
\hline \multicolumn{4}{|l|}{ Probabilities } \\
\hline Pbleeding & probability of having unscheduled bleeding & 0.04 & $0.02-0.05$ \\
\hline PDVT1 & probability of DVT or leg pain due to HRT & .005 & $0.001-0.01$ \\
\hline PGI & probability of having GI disorders & 0.10 & $0.05-0.15$ \\
\hline PDVT2 & probability of DVT or leg pain due to raloxifene & 0.005 & $0.001-0.01$ \\
\hline PcontH & probability of completing 3-year HRT & 0.25 & $0.20-0.30$ \\
\hline PcontA & prob. of completing 3-year alendronate treatment & 0.40 & $0.28-0.52$ \\
\hline PcontR & prob. completing 3-year raloxifene treatment & 0.70 & $0.49-0.91$ \\
\hline
\end{tabular}




\section{PHASE II. ANALYSIS OF BACKGROUND RISK}

Phase II cross-sectional study evaluated the fracture risk in "no therapy" group $\left(\mathrm{I}_{0}\right)$ by estimating the actual risk distribution of the study population.

\section{Subjects}

Results of the data extraction from WV Medicaid claims database are presented in Figure 4-2. The total number of enrolled women aged 45 - 85 and having claims in 19981999 was 37,349 (the mean age was $62.9 \pm 11.7$ years old and $91.3 \%$ were white). Among women aged 45-64 years old $\left(\mathrm{N}_{1}=24,323\right), 431$ women had claims with a diagnosis code of hip, vertebral, or wrist fractures (see Appendix D for related ICD-9 Codes) and all of them were included as sample cases. The sample base for controls $\left(\mathrm{N}_{10}{ }^{\prime}\right)$ was 23,892 , from which 1,000 women were randomly sampled as controls. For women aged 65-85 $\left(\mathrm{N}_{2}=13,026\right)$, the number of women who had been prescribed raloxifene, alendronate, or calcitonin $\left(\mathrm{N}_{20}{ }^{\prime}\right)$, and women who was not on these drugs $\left(\mathrm{N}_{21}{ }^{\prime}\right)$ was 1,295 and 11,731, respectively (see Appendix G for related NDC Codes). In order to have 1000 cases from the two age groups, 569 women were randomly selected from the potential case base. Again, 1000 women were randomly selected from the potential control base. In summary, the overall sample size was 3,000 for the two age groups, including 1,000 possible cases and 2,000 controls. 


\section{Figure 4-2. Results of Data Extraction for the Two Age Groups.}
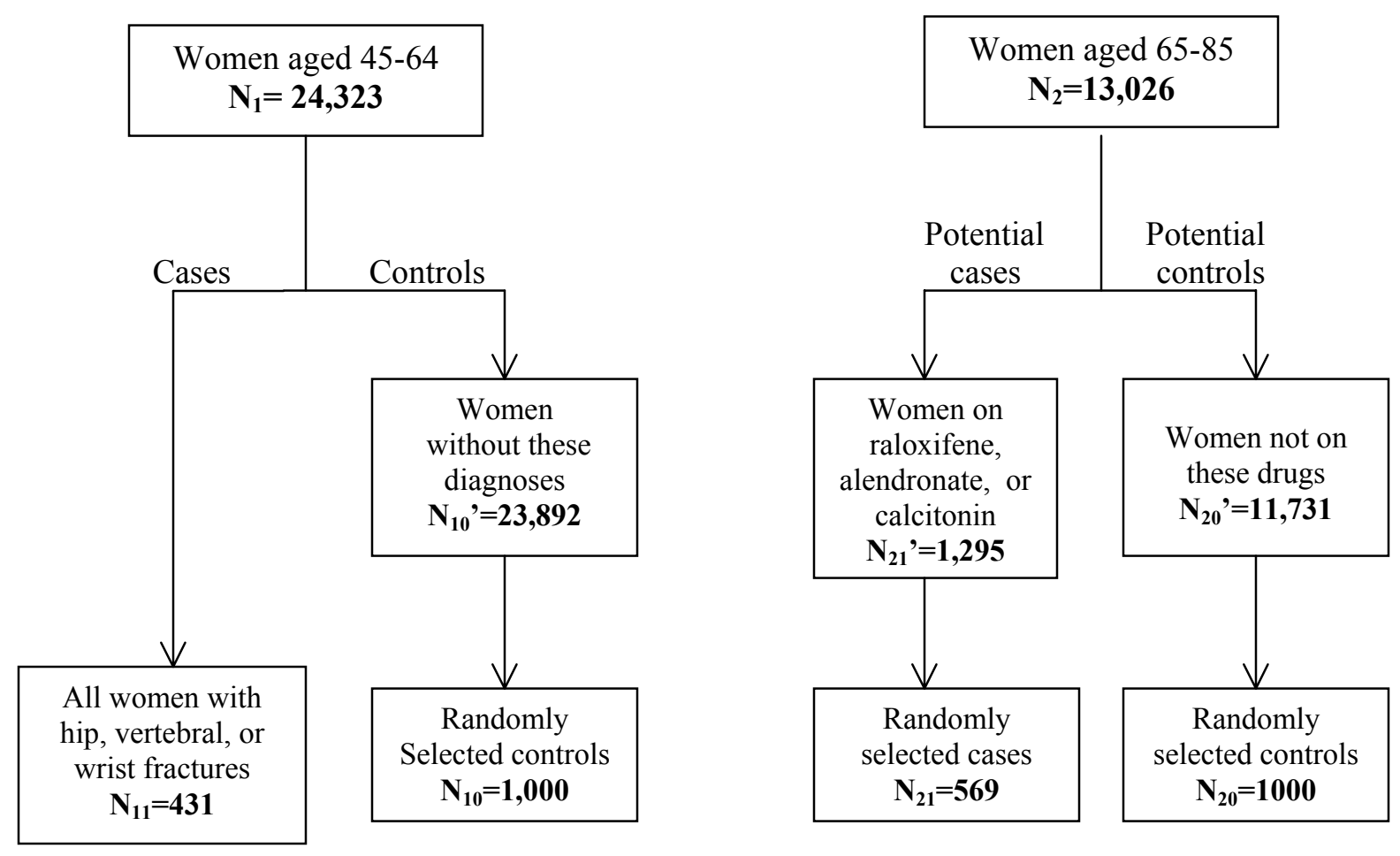
Three thousand surveys were sent out at the first mailing which was followed by a second mailing to the non-respondents of the first mailing after 3 weeks. The total number of responses from the two mailings was 1,466 (cases: 478, controls: 988). The number of surveys returned due to a wrong address or deceased recipients was 273 (cases: 84, controls: 189). The response rates were quite high for both case and control groups: $52.18 \%$ and $54.56 \%$ respectively. However, the eligibility rate of these returned responses was much lower after the inclusion and exclusion criteria were applied (Figure 4-3). Among the 1,466 women who returned the survey, 78 were pre-menopausal (cases: 9; controls: 69); 72 did not answer the question: "Have you ever had an osteoporotic fracture?" (cases: 55; controls: 17); 154 pre-classified cases reported that they had not experienced any osteoporotic fracture, and 121 pre-classified controls reported that they had been diagnosed an osteoporotic fracture; finally, 269 controls indicated that they had taken at least one medication for preventing osteoporotic fracture. After excluding all the above women who met the exclusion criteria, 772 (cases: 260, 34\%; controls: 512, 66\%) out of 1,466 returned responses were eligible for further analysis. The eligibility rate was $54.39 \%$ and $51.82 \%$ for cases and controls, respectively.

Seven cases and 16 controls returned the non-respondent survey (after excluding wrong address or deceased recipients). The most common reasons for not responding the survey were "I did not receive it" ( 2 cases and 5 controls), "I haven't completed it yet" (2 cases and 3 controls); and "I do not respond to mail surveys" ( 1 case and 5 controls). One case and 2 controls indicated that they had already sent the survey back. The mean age of cases and controls were $66.70(n=4)$ and $68.42(n=10)$, respectively. 


\section{Figure 4-3. Results of Data Screening for Cases and Control Groups.}

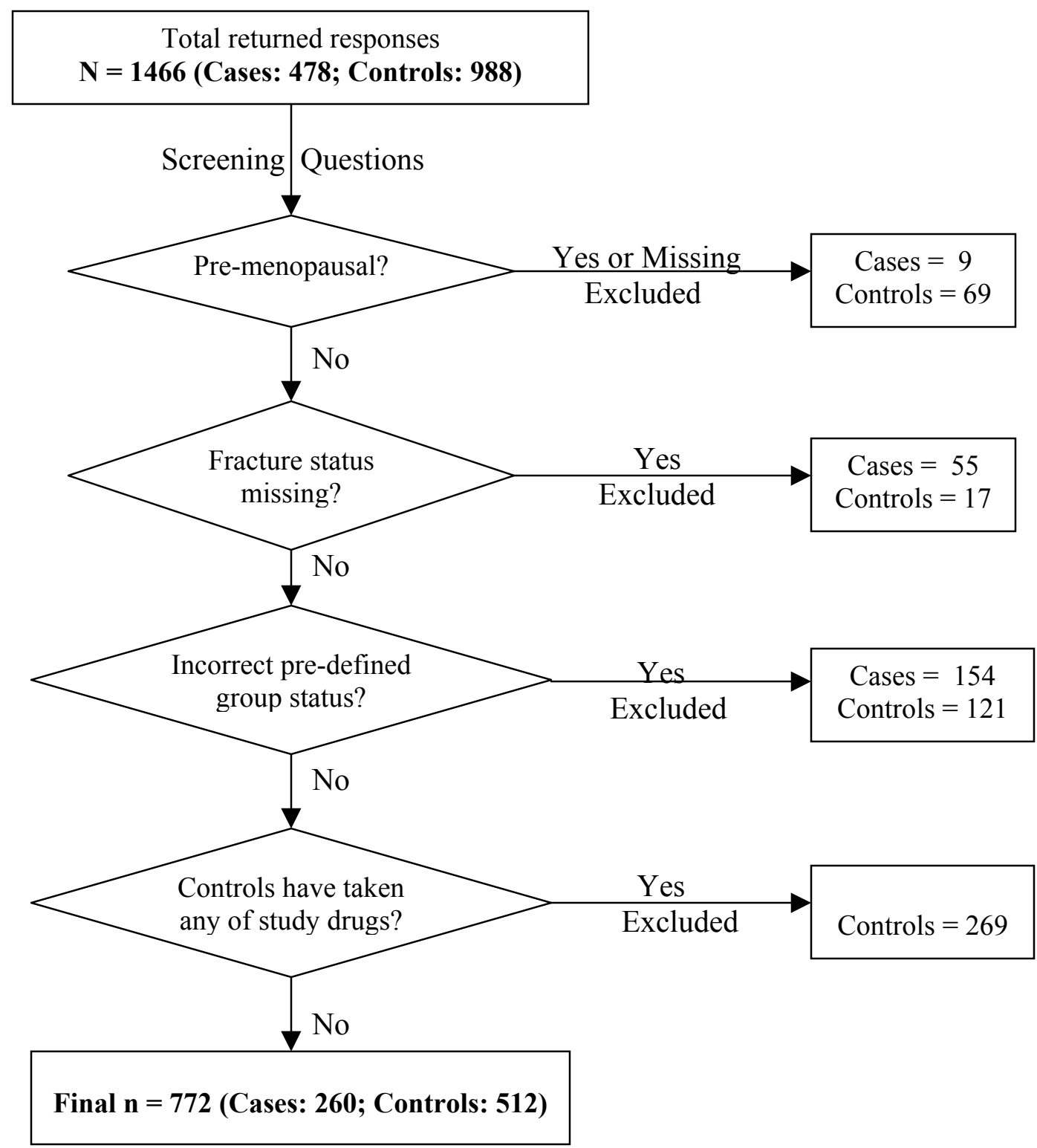

Note: There is a possibility that a woman could meet two or more exclusion criteria and would be excluded by the first one. 


\section{Data Analysis}

Statistical Package for Social Sciences (SPSS-10.0) was used for data analysis. Comparisons on demographic characteristics and health related risk factors between cases and controls are presented in Table 4-10. Overall, the general health status was not good in the study subjects: nearly $50 \%$ of women rated their health as poor, and less than $5 \%$ of women rated their as excellent or very good. Compared to those having osteoporotic fractures, women without osteoporotic fractures reported better general health $\left(X^{2}=\right.$ $31.03, p<.001)$. Women who had female first-degree relative(s) with osteoporosis or osteoporotic fractures accounted for $57 \%$ in cases, compared to $19 \%$ in controls $\left(X^{2}=\right.$ $106.66, p<.001)$. Furthermore, more cases $(47 \%)$ reported their job involved intensive physical activities than controls (35\%). More cases than controls smoked occasionally or everyday $\left(43 \%\right.$ vs. $\left.18 \%, X^{2}=42.46, p<.001\right)$ and drank alcohol $\left(21 \%\right.$ vs. $8.0 \%, X^{2}=$ $25.73, p<.001)$.

There were no statistically significant differences between cases and controls on calcium and multivitamin intake, calcium supplement, exercise, and education. Reported calcium contents of the selected dietary products were used for calculating an average calcium intake (Willhite, 1998; Sagraves et al., 1993; Calcium Supplements, 1989). The majority of all subjects $(60 \%)$ had inadequate calcium intake $(<1200 \mathrm{mg})$. Only a small number of subjects took a calcium supplement $(26 \%)$, vitamin D $(10 \%)$, or did exercises regularly (34\%). The education level of both cases and controls was uniformly low.

Overall, almost $50 \%$ of all subjects had 8 th or less than 8 th grade education, and $95 \%$ of subjects did not go to college. 
Table 11 presents the range and number of valid responses for the cases and controls for the continuous variables. Table 12 provides the means and standard deviations of these continuous variables. The mean age of the study subjects was about $66.5(\mathrm{SD}=10.5)$ years, with $66.3(\mathrm{SD}=10.8)$ years for cases and $66.7(\mathrm{SD}=10.3)$ years for controls. The mean age at menopause was $41.9(\mathrm{SD}=7.8)$ for cases and $46.9(6.4)$ for controls $(F=82.23, p<.001)$. The mean current weight for cases and controls were $145.9(\mathrm{SD}=41.4)$ pounds and $169.3(\mathrm{SD}=45.7)$ pounds, respectively $(F=48.18, p<$ $.001)$. The mean current height for cases and controls were $158.8(\mathrm{SD}=8.3) \mathrm{cm}$ and $160.3(\mathrm{SD}=7.3) \mathrm{cm}$, respectively $(F=6.85, p<.001)$. The current body mass index (BMI) was calculated based on current weight and height. The mean current BMI was significantly lower in cases $\left(26.1 \pm 6.7 \mathrm{~kg} / \mathrm{m}^{2}\right)$ than that in controls $\left(29.8 \pm 7.7 \mathrm{~kg} / \mathrm{m}^{2}\right)(F$ $=42.20, p<.001)$. The mean weight for cases and controls at age of 20-30 years were $129.9(\mathrm{SD}=27.9)$ pounds and $136.4(\mathrm{SD}=30.9)$ pounds, respectively $(F=7.48, p=$ .006). Regarding the height at age of 20-30 years, although the mean current height was significantly higher in controls than that in cases, there was no significant difference in the mean peak height between cases and controls. The mean peak height for cases and controls were $161.9(\mathrm{SD}=6.7) \mathrm{cm}$ and $161.2(\mathrm{SD}=7.2) \mathrm{cm}$, respectively. The peak BMI was also significantly lower in cases $\left(22.4 \pm 4.9 \mathrm{~kg} / \mathrm{m}^{2}\right)$ than that in controls $(23.8 \pm 5.6$ $\left.\mathrm{kg} / \mathrm{m}^{2}\right)(F=9.25, p=.002)$. 
Results

Table 4-10. General Comparisons of Cases and Controls on Categorical Variables.

\begin{tabular}{|c|c|c|c|c|}
\hline Variables & Overall & Cases & Controls & $X^{2}$ \\
\hline \multicolumn{5}{|l|}{ General Health } \\
\hline Excellent & $5(0.7 \%)$ & $1(0.4 \%)$ & $4(0.8 \%)$ & \\
\hline Very good & $29(3.9 \%)$ & $6(2.4 \%)$ & $23(4.7 \%)$ & 31.03 \\
\hline Good & $91(12.2 \%)$ & $16(6.3 \%)$ & $75(15.2 \%)$ & $p<0.001$ \\
\hline Fair & $270(36.3 \%)$ & $77(30.7 \%)$ & $193(39.2 \%)$ & \\
\hline Poor & $348(46.9 \%)$ & $151(60.2 \%)$ & $197(41.0 \%)$ & \\
\hline \multicolumn{5}{|l|}{ Calcium Intake (CI) } \\
\hline $0 \mathrm{mg} \leq \mathrm{CI}<800 \mathrm{mg}$ & $355(48.5 \%)$ & $115(46.7 \%)$ & $240(49.4 \%)$ & \\
\hline $800 \mathrm{mg} \leq \mathrm{CI}<1000 \mathrm{mg}$ & $95(13.0 \%)$ & $32(13.0 \%)$ & $63(13.0 \%)$ & 7.16 \\
\hline $1000 \mathrm{mg} \leq \mathrm{CI}<1200 \mathrm{mg}$ & $63(8.6 \%)$ & $20(8.2 \%)$ & $43(8.8 \%)$ & $p=0.21$ \\
\hline $1200 \mathrm{mg} \leq \mathrm{CI}<1500 \mathrm{mg}$ & $85(11.6 \%)$ & $28(11.4 \%)$ & $57(11.7 \%)$ & \\
\hline $\mathrm{CI} \geq 1500 \mathrm{mg}$ & $134(18.3 \%)$ & $51(20.7 \%)$ & $83(17.1 \%)$ & \\
\hline \multicolumn{5}{|l|}{ Family History } \\
\hline Yes & $231(31.2 \%)$ & $135(56.7 \%)$ & $96(19.1 \%)$ & 106.66 \\
\hline No & $510(68.8 \%)$ & $103(43.3 \%)$ & $407(80.9 \%)$ & $p<0.001$ \\
\hline \multicolumn{5}{|l|}{ Calcium Supplement } \\
\hline Yes & $199(26.1 \%)$ & $66(25.7 \%)$ & $133(26.4 \%)$ & 0.04 \\
\hline No & $562(73.9 \%)$ & $191(74.3 \%)$ & $371(73.6 \%)$ & $p=0.45$ \\
\hline \multicolumn{5}{|l|}{$\underline{\text { Vitamin D }}$} \\
\hline$\overline{\text { Yes }}$ & $78(10.3 \%)$ & $26(10.1 \%)$ & $52(10.4 \%)$ & 0.02 \\
\hline No & $679(89.7 \%)$ & $232(89.9 \%)$ & $447(89.6 \%)$ & $p=0.50$ \\
\hline \multicolumn{5}{|l|}{ Multivitamin Pills } \\
\hline Yes & $163(21.4 \%)$ & $54(21.1 \%)$ & $109(21.6 \%)$ & 0.03 \\
\hline No & $597(78.6 \%)$ & $202(78.9 \%)$ & $395(78.4 \%)$ & $p=0.47$ \\
\hline \multicolumn{5}{|l|}{ Exercise } \\
\hline $\bar{Y}$ Yes & $259(34.0 \%)$ & $92(35.9 \%)$ & $167(33.1 \%)$ & 0.62 \\
\hline No & $502(66.0 \%)$ & $164(64.1 \%)$ & $338(66.9 \%)$ & $p=0.24$ \\
\hline \multicolumn{5}{|l|}{ Physical Job } \\
\hline Yes & $293(39.1 \%)$ & $120(46.7 \%)$ & $173(35.1 \%)$ & 9.55 \\
\hline No & $457(60.9 \%)$ & $137(53.3 \%)$ & $320(64.9 \%)$ & $p=0.001$ \\
\hline
\end{tabular}


Table 4-10. Continued.

\begin{tabular}{lcccc}
\hline \multicolumn{1}{c}{ Variables } & Overall & Cases & Controls & $\boldsymbol{X}^{\mathbf{2}}$ \\
\hline Smoking & & & \\
\hline Never & $310(40.4 \%)$ & $85(33.2 \%)$ & $225(44.0 \%)$ & \\
Quit & $239(31.2 \%)$ & $61(23.8 \%)$ & $178(34.8 \%)$ & 42.46 \\
Occasionally & $31(4.1 \%)$ & $14(5.5 \%)$ & $17(3.4 \%)$ & $p<0.001$ \\
Every day $(<1$ pack) & $100(13.0 \%)$ & $47(18.4 \%)$ & $53(10.4 \%)$ & \\
Every day ( $\geq 1$ pack) & $87(11.3 \%)$ & $49(19.1 \%)$ & $38(7.4 \%)$ & \\
& & & & \\
Alcohol & & & & \\
Never & $529(69.2 \%)$ & $160(62.3 \%)$ & $369(72.6 \%)$ & \\
Quit & $141(18.4 \%)$ & $44(17.1 \%)$ & $97(19.1 \%)$ & 25.73 \\
Occasionally & $79(10.4 \%)$ & $42(16.4 \%)$ & $37(7.3 \%)$ & $p<0.001$ \\
2-3 times a week & $8(1.0 \%)$ & $5(1.9 \%)$ & $3(0.6 \%)$ & \\
Daily/almost daily & $8(1.0 \%)$ & $6(2.3 \%)$ & $2(0.4 \%)$ & \\
& & & & \\
Education & & & & \\
8th or less than 8th grade & $357(46.9 \%)$ & $118(46.1 \%)$ & $239(47.3 \%)$ & \\
9th to 12th grade or GED & $366(48.0 \%)$ & $130(50.8 \%)$ & $236(46.6 \%)$ & 5.38 \\
College 1 year to 3 years & $35(4.6 \%)$ & $6(2.3 \%)$ & $29(5.7 \%)$ & $p=0.15$ \\
College 4 years or more & $4(0.5 \%)$ & $2(0.8 \%)$ & $2(0.4 \%)$ & \\
& & & & \\
\hline
\end{tabular}


Results

Table 4-11. Valid N and Value Range for Continuous Variables

\begin{tabular}{|c|c|c|c|c|c|c|}
\hline \multirow{2}{*}{ Variables } & \multicolumn{2}{|c|}{ Overall } & \multicolumn{2}{|c|}{ Cases } & \multicolumn{2}{|c|}{ Controls } \\
\hline & Valid N & Range & Valid N & Range & Valid N & Range \\
\hline Age (years) & 771 & $45.0-85.0$ & 260 & $45.0-85.0$ & 511 & $45.0-85.0$ \\
\hline Age at menopause & 678 & $20.0-61.0$ & 235 & $20.0-57.0$ & 443 & $26.0-61.0$ \\
\hline Current Weight (pounds) & 765 & $66.0-340.0$ & 260 & $66.0-294.0$ & 505 & $76.0-340.0$ \\
\hline Current Height (cm) & 752 & 121.9-182.9 & 255 & $121.9-176.5$ & 497 & $121.9-182.9$ \\
\hline Current BMI (kg/m²) & 746 & $13.3-54.9$ & 255 & $13.7-51.3$ & 491 & $13.3-54.9$ \\
\hline Peak Weight (pounds) & 679 & $80.0-310.0$ & 242 & $89.0-261.0$ & 437 & $80.0-310.0$ \\
\hline Peak Height (cm) & 653 & 129.5-182.9 & 232 & $142.2-182.9$ & 421 & $129.5-182.9$ \\
\hline Peak BMI (kg/m²) & 619 & $14.4-67.6$ & 222 & $14.4-49.3$ & 397 & $14.6-67.6$ \\
\hline
\end{tabular}


Table 4-12. General Comparisons of Cases and Controls for Continuous Variables.

\begin{tabular}{|c|c|c|c|c|}
\hline Variables & $\begin{array}{c}\text { Overall } \\
(\mathrm{Mean}+\mathrm{SD})\end{array}$ & $\begin{array}{c}\text { Cases } \\
(\mathrm{Mean} \pm \mathrm{SD})\end{array}$ & $\begin{array}{c}\text { Controls } \\
(\mathrm{Mean} \pm \mathrm{SD})\end{array}$ & $F$ \\
\hline Age (years) & $66.54 \pm 10.47$ & $66.27 \pm 10.83$ & $66.68 \pm 10.28$ & 0.26 \\
\hline Age at Menopause & $45.17 \pm 7.34$ & $41.85 \pm 7.81$ & $46.92 \pm 6.42$ & $82.23^{*}$ \\
\hline Current Weight (pounds) & $161.34 \pm 45.61$ & $145.86 \pm 41.41$ & $169.31 \pm 45.66$ & $48.18^{*}$ \\
\hline Current Height $(\mathrm{cm})$ & $159.82 \pm 7.72$ & $158.79 \pm 8.34$ & $160.34 \pm 7.33$ & $6.85^{*}$ \\
\hline Current BMI $\left(\mathrm{kg} / \mathrm{m}^{2}\right)$ & $28.57 \pm 7.60$ & $26.12 \pm 6.74$ & $29.84 \pm 7.72$ & $42.20^{*}$ \\
\hline Peak Weight (pounds) & $134.10 \pm 30.02$ & $129.89 \pm 27.89$ & $136.43 \pm 30.93$ & $7.48^{*}$ \\
\hline Peak Height $(\mathrm{cm})$ & $161.42 \pm 7.03$ & $161.88 \pm 6.73$ & $161.16 \pm 7.18$ & 1.56 \\
\hline Peak BMI $\left(\mathrm{kg} / \mathrm{m}^{2}\right)$ & $23.30 \pm 5.37$ & $22.43 \pm 4.92$ & $23.79 \pm 5.55$ & $9.25^{*}$ \\
\hline
\end{tabular}

${ }^{*}$ The mean difference is significant at $\alpha=0.01$. 
Table 4-13 showed the comorbidities reported by the study subjects. Arthritis was the most prevalent disease, especially in the cases: approximately $79 \%$ of cases and $68 \%$ of controls had arthritis $\left(X^{2}=10.04, p=.001\right)$. Hypertension and diabetes were also quite common, especially in the controls: approximately $50 \%$ of cases and $60 \%$ of controls had hypertension $\left(X^{2}=6.67, p=.006\right)$, and $20 \%$ of cases and $38 \%$ of controls had diabetes $\left(X^{2}=25.28, p<.001\right)$. In addition, $36 \%$ and $23 \%$ of all subjects indicated having heart disease and asthma respectively and there was no significant difference in their reporting between cases and controls. Other diseases reported included allergies, digestive system diseases, chronic obstructive pulmonary disease (COPD), and cancer.

The use of medications that have been reported to affect bone mineral density and the incidence of fractures were also evaluated although the findings on the relationship are still controversial in the literature. The use of steroid and sedatives were significantly higher in cases than that in controls (steroid: $18 \%$ v.s. $11 \%, X^{2}=7.81, p=.001$; sedatives: $22 \%$ v.s. $\left.10 \%, X^{2}=19.69, p<.001\right)$. In contrast, diuretics were used less frequently in cases $\left(17 \%\right.$ v.s. $\left.28 \%, X^{2}=10.56, p<.001\right)$. There was no significant difference in thyroid and antiseizure drugs use between cases and controls. The overall usage for the two drugs was $13 \%$ and $7 \%$, respectively. 
Table 4-13. Comorbidity and the Use of Drugs that May Affect Osteoporotic Fracture Incidence among Cases and Controls.

\begin{tabular}{lcccc}
\hline \multicolumn{1}{c}{ Variables } & Overall & Cases & Controls & $\boldsymbol{X}^{\mathbf{2}}$ \\
\hline Comorbidity & & & \\
\hline Arthritis & $553(71.6 \%)$ & $205(78.8 \%)$ & $348(68.0 \%)$ & $10.04^{*}$ \\
Asthma & $174(22.5 \%)$ & $68(26.2 \%)$ & $106(20.7 \%)$ & 2.93 \\
Diabetes & $249(32.3 \%)$ & $53(20.4 \%)$ & $196(38.3 \%)$ & $25.28^{*}$ \\
Hypertension & $433(56.1 \%)$ & $129(49.6 \%)$ & $304(59.4 \%)$ & $6.67^{*}$ \\
Heart disease & $278(36.0 \%)$ & $102(39.2 \%)$ & $176(34.4 \%)$ & 1.76 \\
& & & & \\
Affecting Drugs & $100(13.0 \%)$ & $46(17.7 \%)$ & $54(10.5 \%)$ & $7.81^{*}$ \\
\hline Steroid & $150(19.4 \%)$ & $49(18.8 \%)$ & $101(19.7 \%)$ & 0.09 \\
Thyroid & $188(24.4 \%)$ & $45(17.3 \%)$ & $143(27.9 \%)$ & $10.56^{*}$ \\
Diuretics & $109(14.1 \%)$ & $57(21.9 \%)$ & $52(10.2 \%)$ & $19.69^{*}$ \\
Sedatives & $55(7.1 \%)$ & $21(8.1 \%)$ & $34(6.6 \%)$ & 0.54 \\
Antiseizure & & & & \\
\hline
\end{tabular}

* The group difference is significant at $\alpha=0.05$. 


\section{$\underline{\text { Building and Validating a Risk Prediction Model }}$}

A multiple logistic regression model was used to investigate the influence of age, age at menopause, family history, physical job, smoking, alcohol use, and current BMI, while controlling for their general health and some medications use. Other possible factors such as calcium intake and exercise were not included in the model based on the results of general comparison between cases and controls. Additionally, due to a large number of missing data and relatively higher recall bias, the peak BMI calculated from peak weight and peak height (weight and height at age of 20 - 30 years) was excluded from the regression model. Comparison of the data in a bivariate correlation matrix revealed no evidence of a problematic level of multicolinearity among predictors. All Pearson correlation coefficients among predictors were $<0.50$ (Licht, 1994).

Results of the multiple regression analysis including model coefficients, odds ratios (ORs), and related statistics are presented in Table 4-14. The analysis revealed that family history, current BMI, age at menopause, self-rated health, and diuretics use were significantly related to the occurrence of predictors of osteoporotic fracture. Family history was the most powerful predictor of osteoporotic fracture $(\mathrm{OR}=8.49, p<.001)$. Poor self-rated health was also associated with having a fracture $(\mathrm{OR}=1.56, \mathrm{p}=0.016)$. The probability of fracture was negatively related to the current $\mathrm{BMI}(\mathrm{OR}=0.93, p=$ $.002)$, age at menopause $(\mathrm{OR}=0.89, p<.001)$, and the use of diuretics $(\mathrm{OR}=0.44, p=$ .045). Age, the use of steroid and sedatives, alcohol consumption, smoking, and physical job did not show a significant relationship with fractures. The goodness of fit $\mathrm{X}^{2}$ test was $135.84(p<.001)$ for the risk assessment model $\left(-2 \mathrm{LL}=275.81, \mathrm{R}^{2}=0.48\right)$. 
Table 4-14. The Relationship of Osteoporotic Fractures and Selected Risk Factors

\begin{tabular}{lcccccc}
\hline $\begin{array}{l}\text { Risk Factors \& } \\
\text { Controlled Variables }\end{array}$ & B & S.E. & Wald & $\boldsymbol{p}$ & OR & 95\% CI \\
\hline Family history & 2.13 & .34 & 39.73 & $<.001$ & 8.49 & $(4.37,16.51)$ \\
Current BMI & -.08 & .02 & 9.92 & .002 & .93 & $(.89, .97)$ \\
Age at menopause & -.12 & .02 & 23.97 & $<.001$ & .89 & $(.85, .93)$ \\
Self-rated health & .45 & .19 & 5.79 & .016 & 1.56 & $(1.09,2.24)$ \\
Diuretics & -.82 & .41 & 4.01 & .045 & .44 & $(.20, .98)$ \\
Steroid & .79 & .46 & 2.91 & .088 & 2.21 & $(.89,5.47)$ \\
Sedatives & .46 & .41 & 1.31 & .252 & 1.59 & $(.72,3.52)$ \\
Alcohol use & .27 & .20 & 1.93 & .165 & 1.31 & $(.89,1.92)$ \\
Smoking & .19 & .126 & 2.70 & .101 & 1.21 & $(.96,1.52)$ \\
Physical job & -.13 & .31 & .17 & .681 & .88 & $(.47,1.63)$ \\
Age & .03 & .02 & 2.63 & .105 & 1.03 & $(1.00,1.06)$ \\
\hline
\end{tabular}

* For all scales, higher scores represent greater amounts of the construct or the presence of the disease.

** Logistic Regression Model: $X^{2}=135.84, p<0.001,-2 \mathrm{LL}=275.81, \mathrm{R}^{2}=0.48$ 
The predicted probability of having an osteoporotic fracture was saved as a new variable. Since the case-to-control ratio was 1:2, a cutoff value of 0.66 (66\% probability or chance) was applied to predict the group membership which was also saved as a new variable with a value of 0 or $1(0=$ non-fracture group; $1=$ fracture group $)$. Table 4-15 shows that the sensitivity of the prediction model was $44 \%$ and the specificity was $95 \%$. The positive predictive value (PPV) and negative predictive value (NPV) were approximately $81 \%$ and $77 \%$, respectively. The false positive rate (FPR) and false negative rate (FNR) were approximately $19 \%$ and $23 \%$, respectively. The overall percentage accuracy classification (PAC) was $78 \%$. Area under the ROC curve $=0.85$ $(\mathrm{p}<.001,95 \%$ CI: .81 - .88) (Figure 4-4 \& Table 4-16).

To validate the model generated in logistic regression above, the data set were split as two subsets by randomly selecting $50 \%$ of cases. Half of the subjects (selected cases) were used in model estimation, and the other half (unselected cases) was used for the model validation. Model validation results are presented in Table 4-17. The results showed that the key statistics were fairly consistent or repeatable in the unselected cases. The specificity and the overall prediction accuracy in the selected cases kept relatively high. The sensitivity and the PPV, NPV, FPR, and FNR were also confirmed in the unselected cases (Table 4-18). 
Table 4-15. Prediction Power of the Result Model Based on Selected Cases (N=323).

\begin{tabular}{lc}
\hline \multicolumn{1}{c}{ Measures } & Rates \\
\hline Sensitivity & $43.5 \%$ \\
Specificity & $94.9 \%$ \\
Positive Predictive Value (PPV) & $81.0 \%$ \\
Negative Predictive Value (NPV) & $77.0 \%$ \\
False Positive Rate & $19.0 \%$ \\
False Negative Rate & $23.0 \%$ \\
Overall Accuracy & $77.7 \%$ \\
\hline
\end{tabular}


Results

Figure 4-4.

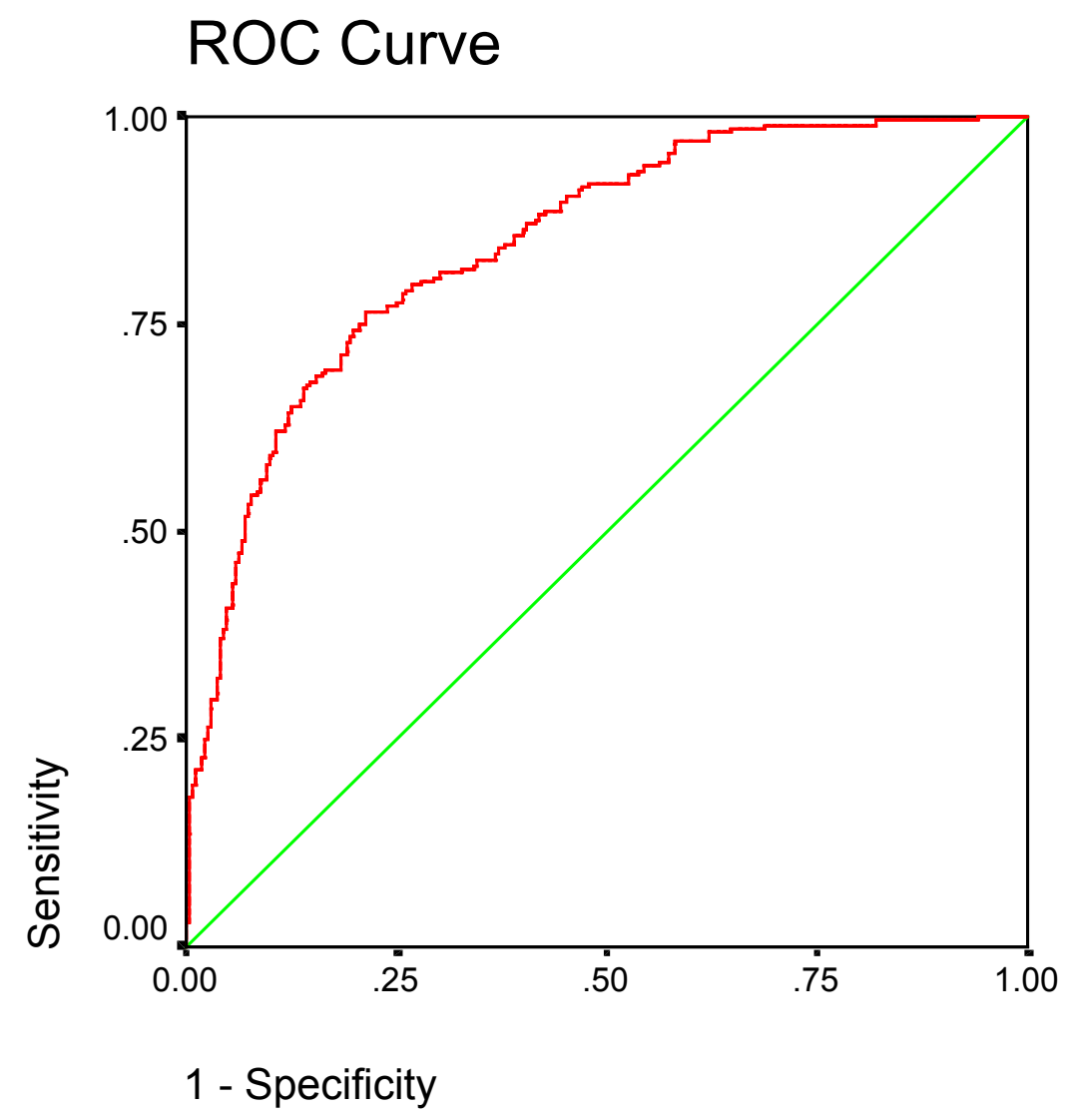


Table 4-16. Area Under the Curve (Test Result Variable: Predicted Probability)

\begin{tabular}{cccc}
\hline Area & Std. Error & Asymptotic Sig. & $95 \% \mathrm{CI}^{*}$ \\
\hline $8 \dot{ }$ & .016 & .000 & $.814-.878$ \\
\hline
\end{tabular}

* CI: Confidence Interval 
Table 4-17. Results of Model Validation with the 50\% Hold-Out Sample.

\begin{tabular}{|c|c|c|c|c|c|c|}
\hline \multirow{3}{*}{ Observed } & \multicolumn{6}{|c|}{ Predicted } \\
\hline & \multicolumn{3}{|c|}{ Selected Cases } & \multicolumn{3}{|c|}{ Unselected Cases } \\
\hline & \begin{tabular}{|c}
$\begin{array}{c}\text { Non-fracture } \\
\text { group }\end{array}$ \\
\end{tabular} & $\begin{array}{c}\text { Fracture } \\
\text { group }\end{array}$ & \begin{tabular}{|c|}
$\begin{array}{c}\text { Percentage } \\
\text { Correct }\end{array}$ \\
\end{tabular} & $\begin{array}{c}\begin{array}{c}\text { Non-fracture } \\
\text { group }\end{array} \\
\end{array}$ & $\begin{array}{c}\text { Fracture } \\
\text { group }\end{array}$ & \begin{tabular}{|c|} 
Percentage \\
Correct
\end{tabular} \\
\hline $\begin{array}{l}\text { Non-fracture } \\
\text { group }\end{array}$ & 204 & 11 & 94.9 & 161 & 14 & 92.0 \\
\hline $\begin{array}{l}\text { Fracture } \\
\text { group }\end{array}$ & 61 & 47 & 43.5 & 45 & 48 & 51.6 \\
\hline $\begin{array}{l}\text { Overall } \\
\text { Percentage }\end{array}$ & & & 77.7 & & & 78.0 \\
\hline
\end{tabular}
a. $\mathrm{N}_{\text {selected cases }}=323$ (41.8\%); $\mathrm{N}_{\text {unselected cases }}=354(45.9 \%) ; \mathrm{N}_{\text {missing }}=95(12.3 \%)$.
b. Some cases are not classified due to missing values in the independent variables.
c. The probability cut value is 0.66 for the membership prediction. 
Table 4-18. Prediction Power of the Result Model Based on the Hold-out Sample.

\begin{tabular}{lc}
\hline \multicolumn{1}{c}{ Measures } & Rates \\
\hline Sensitivity & $51.6 \%$ \\
Specificity & $92.0 \%$ \\
Positive Predictive Value (PPV) & $77.4 \%$ \\
Negative Predictive Value (NPV) & $78.2 \%$ \\
False Positive Rate & $22.6 \%$ \\
False Negative Rate & $21.8 \%$ \\
Overall Accuracy & $78.0 \%$ \\
\hline
\end{tabular}




\section{Estimating Risk Distributions in the Study Control Sample}

The distribution of predicted risk in the study sample was evaluated. The study control sample referred to the WV Medicaid white women, aged 45 - 85 years, postmenopausal, without past incidence of osteoporotic fractures, and not taking any of the study drugs. Necessary information of 122 women on their predicted risk or probability and group membership was missing due to missing value(s) in the risk factor(s). Therefore, the final valid study sample size is 390 . The estimated risk distribution in the study sample is presented in Figure 4-5.

The mean risk was $22.0 \%(\mathrm{SD}=22 \%)$. The lowest probability of fracture was $0.37 \%$ and the highest probability was $98 \%$ in the study sample. One quarter of the sample had fracture risk less than $5.3 \%$, half of the sample had risk less than $13 \%$, and the majority (75\%) of the sample had risk less than $31 \%$.

The risk or probability of osteoporotic fracture was further categorized into three levels: low (risk $<0.1)$, medium $(0.1 \leq$ risk $<0.3)$, and high (risk $\geq 0.3)$. Statistics for the overall risk distribution and the distribution in each risk category are shown in Table 419. One hundred and sixty-six women ( $42.6 \%$ of the total sample) were classified into low risk group, with a mean risk of $5 \%(\mathrm{SD}=2.7 \%)$. Most women in this low risk group had a fracture risk less than $7 \%$. One hundred and twenty-three women $(31.5 \%$ of the total sample) were classified into medium risk group, with a mean risk of $19 \%$ (SD = $5.9 \%$ ). Seventy-five percent of women in this group had a fracture risk less than $24 \%$. The rest of the women in the study sample $(101,25.9 \%)$ fell in the high risk group with a mean risk of $53 \%(\mathrm{SD}=18 \%)$. Almost half of women in this group had a fracture risk or 
probability greater than $50 \%$ (who would be classified into fracture group by the prediction model based on the predicted probability).

Since the study control sample was randomly selected from the study population, the fracture probability distribution in this sample was inferred to all the fracture-free women (and without taking any of the study drugs) in the study population. 
Figure 4-5. Risk Distribution in the Study Control Sample $(\mathrm{N}=390)$.

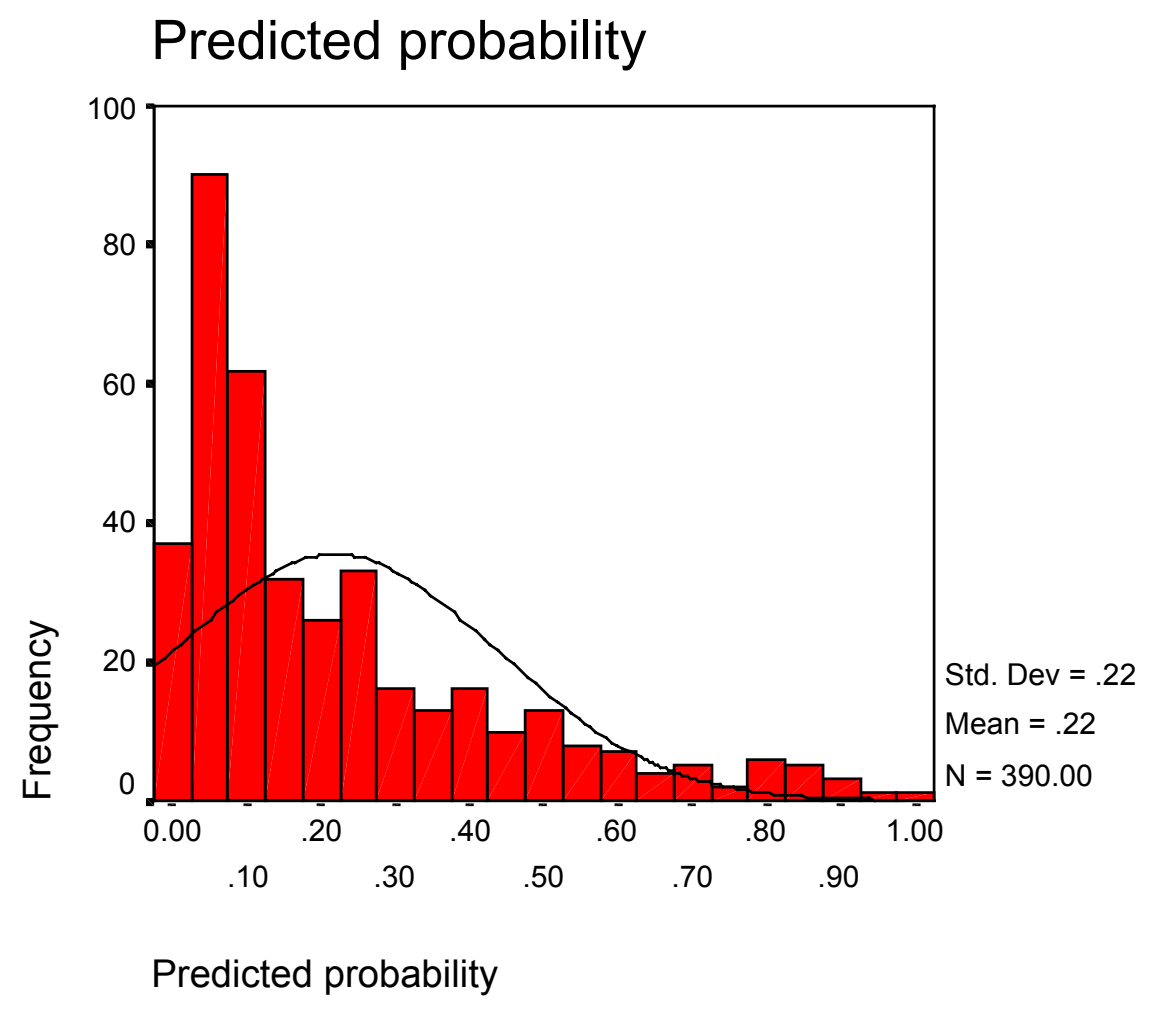


Table 4-19. Overall and Categorized Risk Distributions in the Study Control Sample.

\begin{tabular}{ccccc}
\hline & All Risk Levels & $\begin{array}{c}\text { Low Risk } \\
(0 \leq \text { risk }<0.1)\end{array}$ & $\begin{array}{c}\text { Medium Risk } \\
(0.1 \leq \text { risk }<0.3)\end{array}$ & $\begin{array}{c}\text { High Risk } \\
(0.3 \leq \text { risk }<1)\end{array}$ \\
\hline Valid N (\%) & $390(100)$ & $166(42.56)$ & $123(31.54)$ & $101(25.90)$ \\
Mean + SD & $0.22 \pm 0.22$ & $0.050 \pm 0.027$ & $0.19 \pm 0.059$ & $0.53 \pm 0.18$ \\
Range & $0.0037-0.98$ & $0.0037-0.099$ & $0.10-0.30$ & $0.30-0.98$ \\
Percentiles & & & & 0.39 \\
25 & 0.053 & 0.027 & 0.14 & 0.49 \\
50 & 0.13 & 0.048 & 0.19 & 0.66 \\
75 & 0.31 & 0.073 & 0.24 & \\
\hline
\end{tabular}




\section{Phase III. Cost-Effectiveness Analysis}

In order to evaluate the risk-based cost-effectiveness of HRT, alendronate, and raloxifene, Phase III study applied the background risk distribution estimated in Phase II to the decision model built in Phase I.

\section{Cost-Effectiveness Analysis Using Mean Values}

First, an analysis was conducted using the "Roll Back" method to calculate CE ratios. The base value of the direct medical costs and risk reduction rate obtained from Phase I, and the mean risk of a woman without any interventions estimated from Phase II were used in the "Roll Back" procedure. The results are presented in Table 4-20.

The results showed that HRT had the lowest costs $(\$ 885)$, while the raloxifene treatment had the highest effectiveness (0.12). The cost-effectiveness (CE) of HRT, alendronate, and raloxifene were $\$ 12,401, \$ 16,082$, and $\$ 18,685$ per fracture avoided, respectively. The marginal or incremental cost-effectiveness was $\$ 23,799$ for alendronate relative to HRT, and $\$ 37,782$ for raloxifene relative to alendronate.

Simple sensitivity analyses in which variables were tested individually were utilized to evaluate the robustness of the model. The highest and lowest possible values for each cost, effectiveness, probability, and compliance variable were tested in the model (see Table 4-9 in Phase I results). 
Table 4-20. Results of Cost-Effectiveness Analysis Using Mean Values.

\begin{tabular}{lcccccc}
\hline Strategy & $\begin{array}{c}\text { Cost } \\
(\$)\end{array}$ & $\begin{array}{c}\text { Marginal Effectiveness } \\
\text { Cost } \mathbf{( \$ )}\end{array}$ & $\begin{array}{c}\text { Marginal } \\
\text { Effectiveness }\end{array}$ & $\mathbf{C / E}$ & $\begin{array}{c}\text { Marginal } \\
\mathbf{C} / \mathbf{E}\end{array}$ \\
\hline HRT & 884.70 & & 0.0713 & & $12,401.11$ & \\
Alendronate & $1,694.70$ & 810.00 & 0.1054 & 0.0341 & $16,082.36$ & $23,798.71$ \\
& & & & & & \\
Raloxifene & $2,237.40$ & 542.70 & 0.1197 & 0.0144 & $18,685.40$ & $37,782.25$ \\
\hline
\end{tabular}


Results from a sensitivity analysis on costs revealed that even when HRT costs were increased by $10 \%$, it still was the most cost-effective treatment (Figure 4-6). When the costs of alendronate therapy changed from $\$ 1,525$ to $\$ 1,864( \pm 10 \%$ on the base value), the CE increased from $\$ 14,471$ to $\$ 17,687$. Raloxifene CE ranged from $\$ 16,823$ to $\$ 20,561$ with the $10 \%$ variation in costs. Raloxifene therapy was more cost-effective than alendronate when its costs were reduced by at least $10 \%$.

The CE of HRT, alendronate, and raloxifene was sensitive to the risk reduction rate (RR) of each therapy (Figure 4-7). CE ratios varied greatly with the change of risk reduction rates: the CE of HRT was tripled (from \$8,267 to \$24,802) when the RR decreased from 0.6 to 0.2 , and the $\mathrm{CE}$ of raloxifene was doubled (from $\$ 15,373$ to $\$ 30,747)$ when the RR decreased from 0.6 to 0.3 . The change in alendronate CE was relatively moderate compared to the other two alternatives: the $\mathrm{CE}$ increased from $\$ 13,052$ to $\$ 18,853$ when the RR decreased from 0.65 to 0.45 .

Table 4-21 shows that the CE of HRT, alendronate, raloxifene was not sensitive to those AE probabilities. Women's willingness-to-continue rate (WTC) did not influence the CE of HRT and alendronate although it had a moderate effect on the CE of raloxifene (Figure 4-8). 


\section{Figure 4-6. Sensitivity Analysis on Treatment Costs}

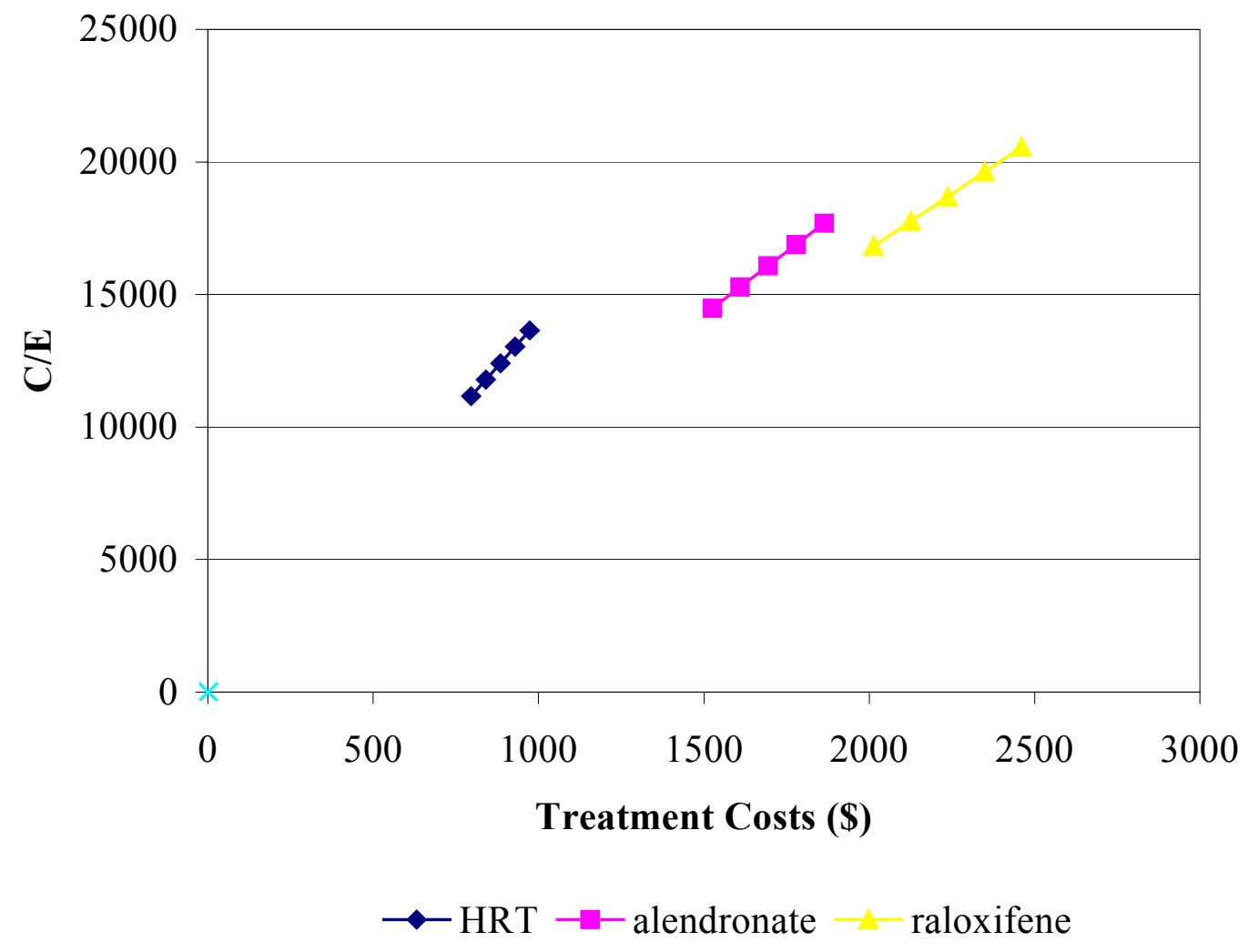


Figure 4-7. Sensitivity Analysis on Risk Reduction Rate

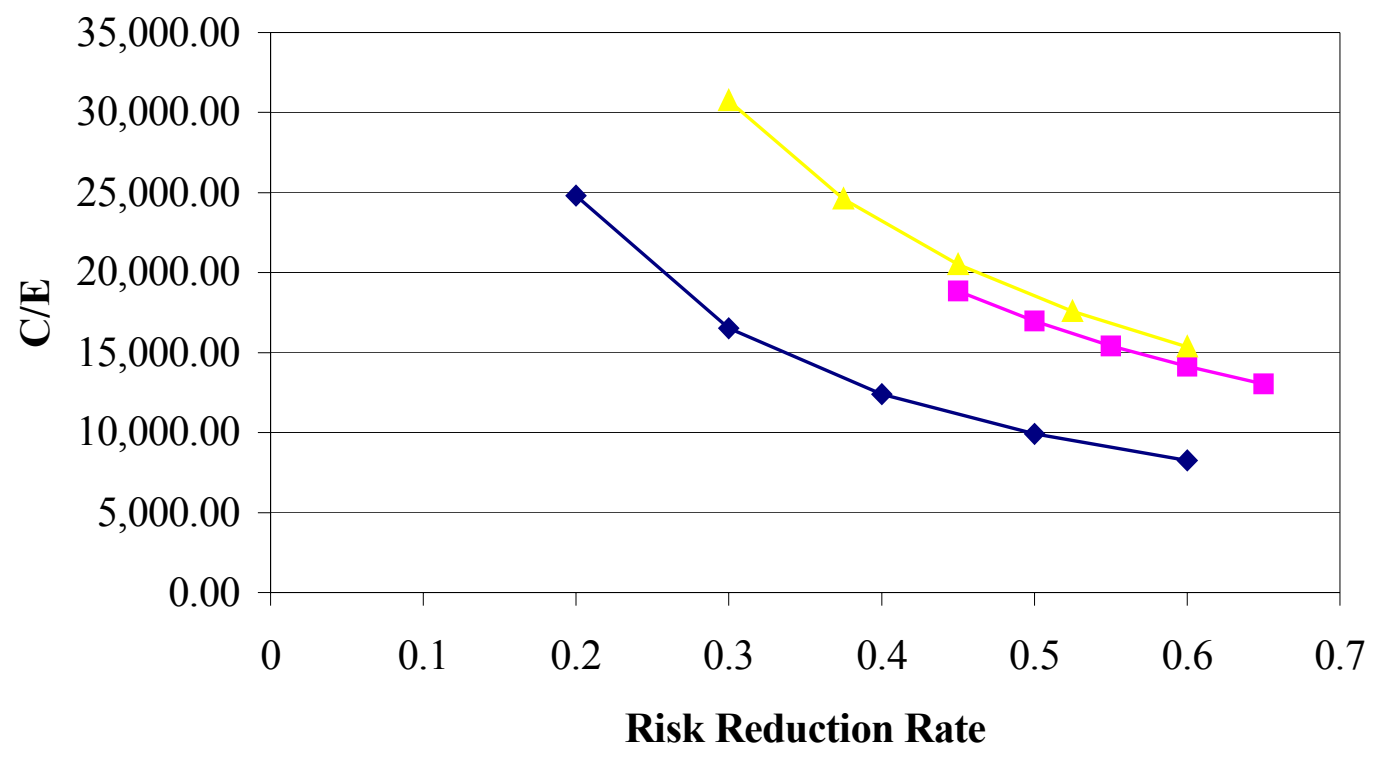

$\multimap$ HRT $\rightarrow-$ alendronate $\longrightarrow$ raloxifene 
Table 4-21. Sensitivity Analysis on AE probability and WTC Rate.

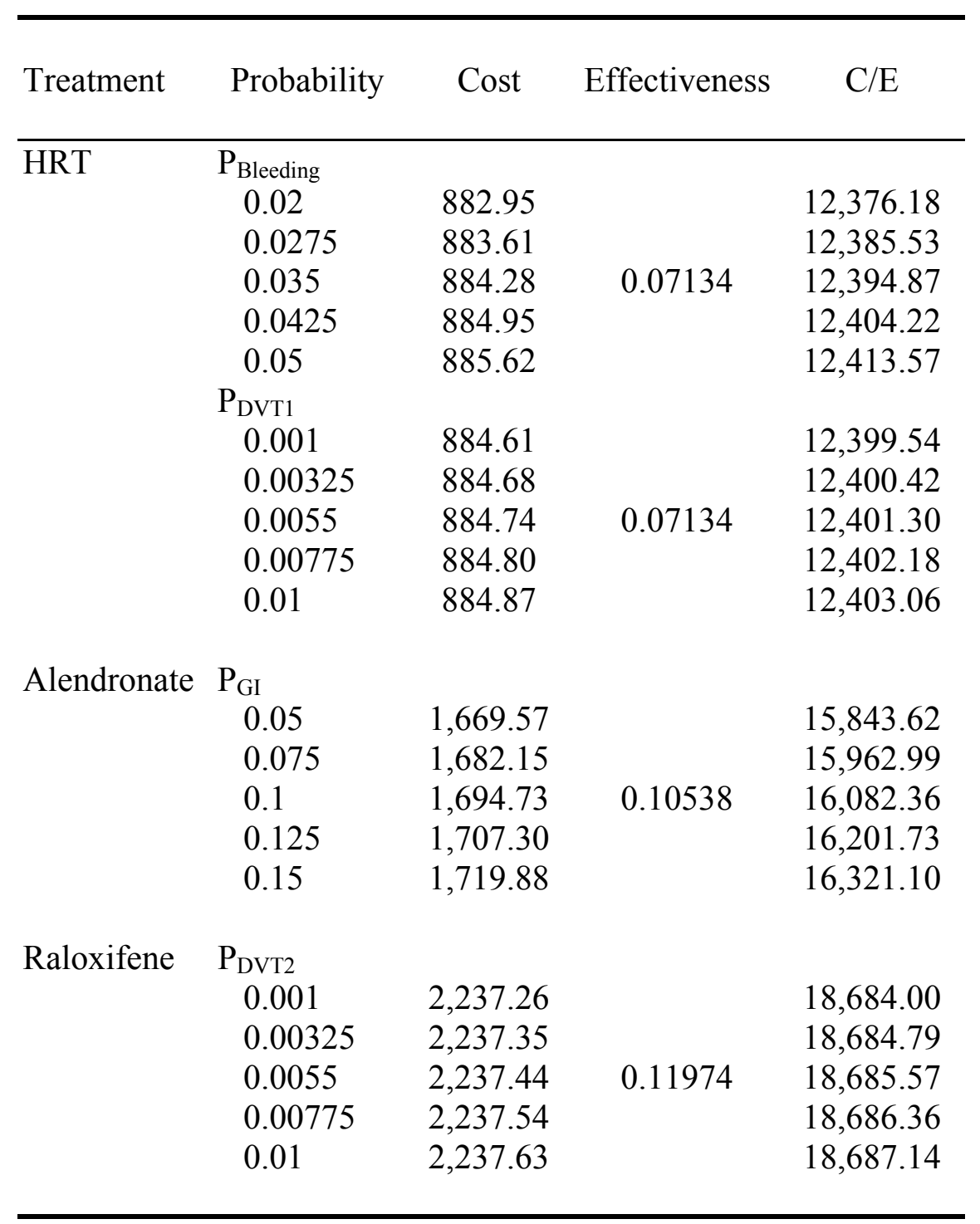


Figure 4-8. Sensitivity Analysis on WTC Rate

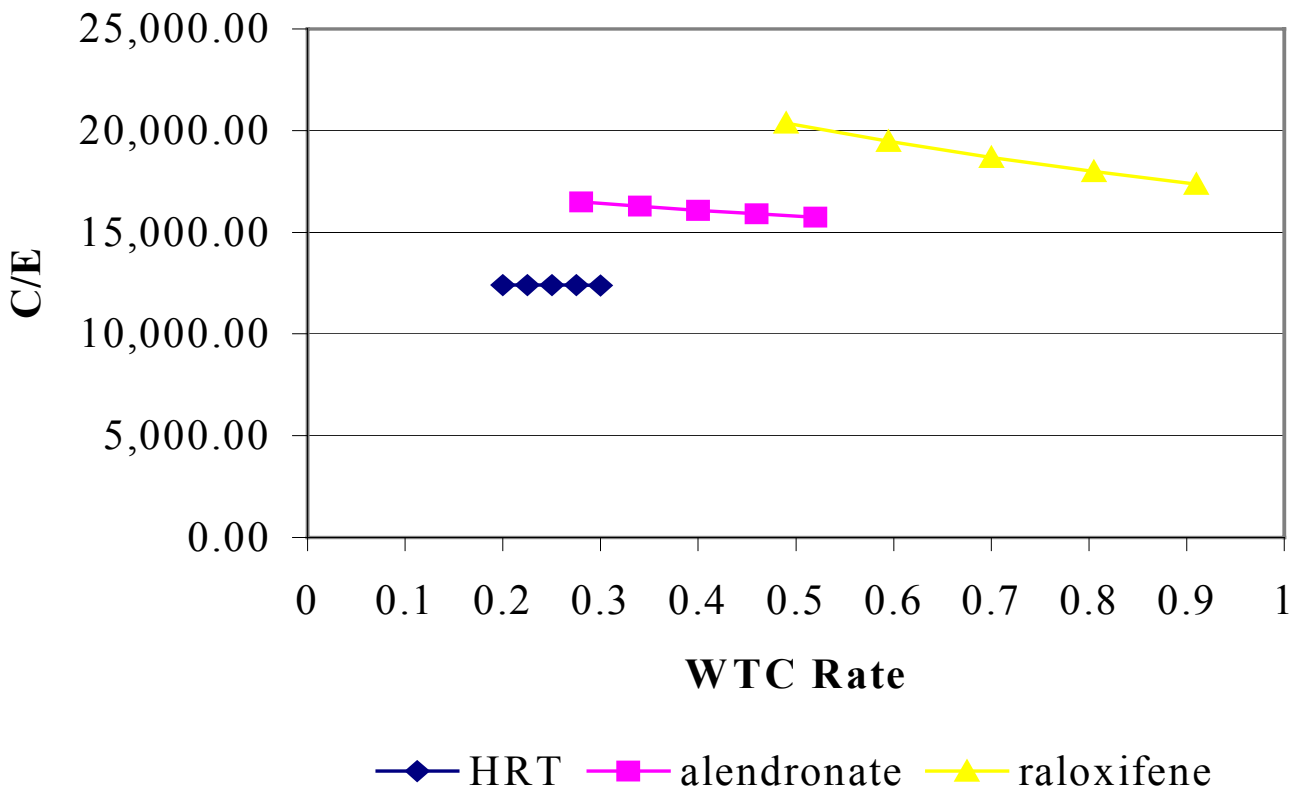




\section{Probabilistic Analysis and Monte Carlo Simulation}

In probabilistic analysis, distribution variables, instead of single values, are used.

Based on the results of simple sensitivity analyses, new distribution variables were created for treatment costs, risk reduction rate, and background risk. The distributions of treatment costs and fracture reduction rates were estimated by Triangle distribution using high, basic, and low values. The distribution of background risk was estimated from the actual risk distribution in the study sample obtained from Phase II. The raw data table was used in the simulation since no preset distributions in Treeage program fits the actual distribution.

Monte Carlo simulation was conducted incorporating all the randomly generated values from the distributions of input variables. The number of simulations (i.e., the number of values randomly sampled from each distribution) was set to 500. The new decision tree structure using distribution variables for Monte Carlo simulations is demonstrated in Figure 4-9. 
Figure 4-9. Decision Tree Structure Using Distribution Variables

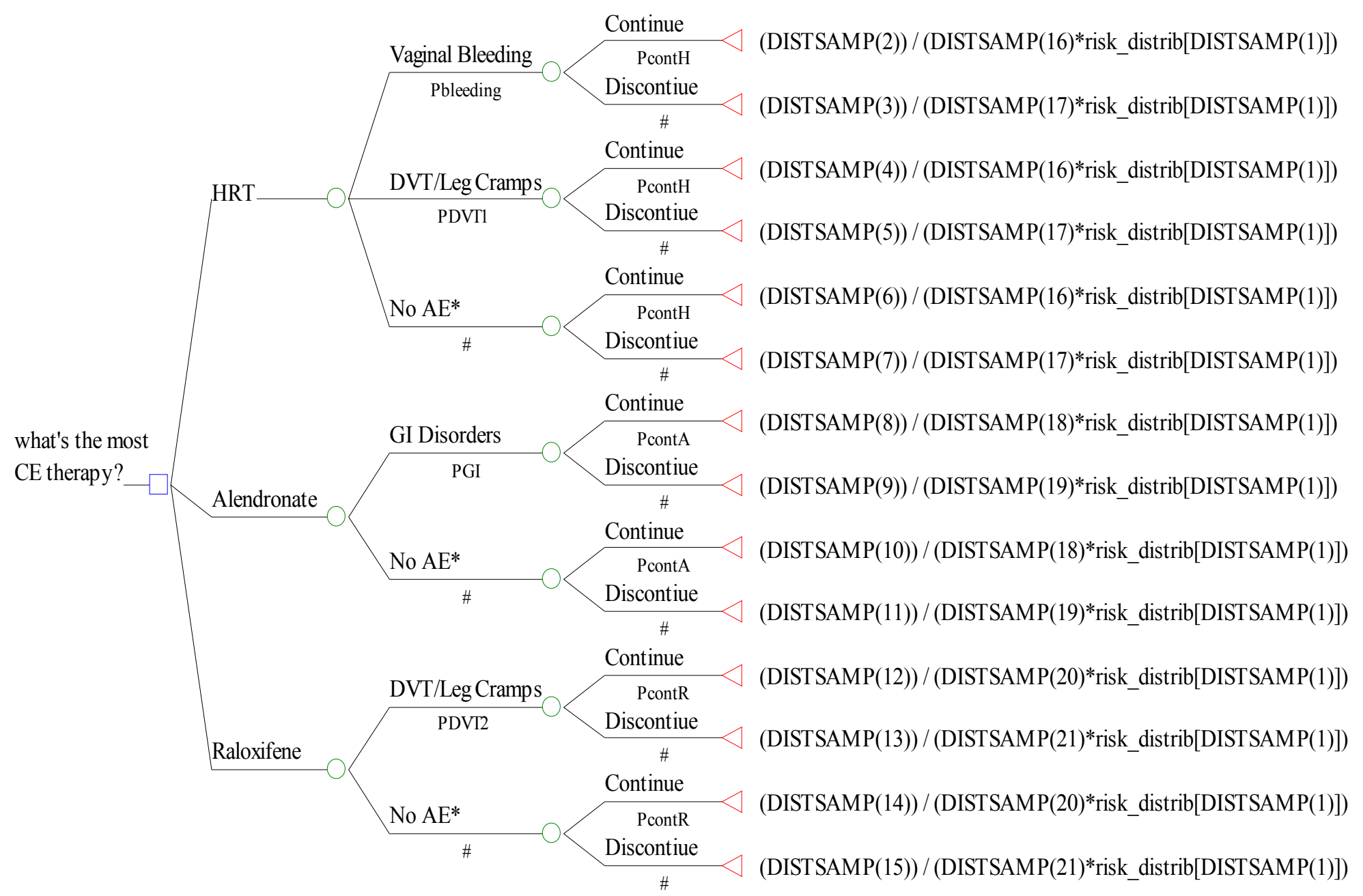


Results of Monte Carlo simulations in the overall study sample showed that compared to no therapy, the expected CE was $\$ 29,119$ per fracture averted for HRT, $\$ 35,101$ for alendronate, and $\$ 39,760$ for raloxifene (Table 4-22). The incremental or marginal CE was $\$ 42,181$ for alendronate relative to HRT and more than doubled $(\$ 85,509)$ for raloxifene relative to alendronate.

In order to evaluate how cost-effectiveness of each drug therapy was affected by the fracture risks in the study population, CE ratios were also calculated among women with different risk levels. The overall risk distribution variable was replaced by 3 distribution variables reflecting 3 risk levels (see Table 4-19): low (risk $<0.1$ ), medium $(0.1 \leq$ risk $<0.3)$, and high (risk $\geq 0.3)$. The same Monte Carlo simulation procedure was run for each stratified risk distribution variable. The results are presented in a matrix of expected CE ratios by risk levels (Table 4-23). For all three therapies, CE declined rapidly with increased fracture risk. For women with low fracture risk, the cost of preventing one fracture was $\$ 79,130, \$ 105,911$, and $\$ 140,675$ for HRT, alendronate, and raloxifene, respectively. For women with medium and high fracture risks, the costs for preventing one fracture significantly decreased for all three therapies, especially for alendronate and raloxifene. The CE was $\$ 19,569, \$ 25,937$, and $\$ 30,505$ for HRT, alendronate, and raloxifene, respectively in women with medium risk, compared to $\$ 7,232, \$ 9,690$, and $\$ 11,536$ respectively in women with high risk. The incremental CE of alendronate (relative to HRT) and raloxifene (relative to alendronate) were $\$ 151,981$ and $\$ 697,270$ among women with low risk, compared to only $\$ 11,099$ and $\$ 34,017$ respectively among high-risk women. 
Table 4-22. Cost-Effectiveness Ratios of the Three Drug Therapies in the Whole Study Sample*.

\begin{tabular}{lcccccc}
\hline Strategy & $\begin{array}{c}\text { Cost } \\
(\$)\end{array}$ & $\begin{array}{c}\text { Marginal Effectiveness } \\
\text { Cost (\$) }\end{array}$ & $\begin{array}{c}\text { Marginal } \\
\text { Effectiveness }\end{array}$ & $\mathbf{C} / \mathbf{E}$ & $\begin{array}{c}\text { Marginal } \\
\mathbf{C} / \mathbf{E}\end{array}$ \\
\hline HRT & 717.34 & & 0.0296 & & $29,118.55$ & \\
Alendronate & 1392.65 & 675.31 & 0.0456 & 0.0160 & $35,101.03$ & $42,180.51$ \\
& & & & & & \\
Raloxifene & 2335.81 & 943.16 & 0.0567 & 0.0111 & $39,760.47$ & $85,508.61$ \\
\hline
\end{tabular}

* Median values (Expected Values) of cost, effectiveness, and C/E were used. 
Table 4-23. Cost-Effectiveness Ratios of the Three Drug Therapies in Women with Different Risk Levels*

\begin{tabular}{|c|c|c|c|c|c|c|}
\hline $\begin{array}{l}\text { Risk } \\
\text { Levels }\end{array}$ & Low & Risk & Medi & Risk & Hig & Risk \\
\hline Strategy & $\mathrm{CE}$ & $\begin{array}{c}\text { Marginal } \\
\mathrm{CE}\end{array}$ & $\mathrm{CE}$ & $\begin{array}{c}\text { Marginal } \\
\mathrm{CE}\end{array}$ & $\mathrm{CE}$ & $\begin{array}{c}\text { Marginal } \\
\mathrm{CE}\end{array}$ \\
\hline HRT & $79,130.02$ & & 19.569 .40 & & $7,232.48$ & \\
\hline Alendronate & $105,910.69$ & $151,981.2$ & $25,937.43$ & $35,256.7$ & $9,690.24$ & $11,098.7$ \\
\hline Raloxifene & $140,675.34$ & $697,270.1$ & $30,505.01$ & 75,714 & $11,535.96$ & $34,016.8$ \\
\hline
\end{tabular}

* Median values (Expected Values) of C/E was used. 


\section{CHAPTER 5}

\section{DISCUSSIONS AND RECOMMENDATIONS}

This chapter presents a review of the study and discusses its major findings. Where applicable, conclusions are drawn regarding those findings. In addition, the implications of this study are stated. Finally, the major study limitations are discussed and recommendations for the future research are made.

\section{Study Review}

Osteoporosis is an important health problem in the United States affecting approximately 15 to 20 million women over 45 years of age (Iqbal, 2000). Bone fractures are the major cause of morbidity and mortality associated with osteoporosis. Osteoporotic fractures result in complications leading to prolonged hospitalization, decreased independence, increased morbidity and mortality, and reduced quality of life. The disease takes an enormous personal and economic toll, with estimated costs in excess of $\$ 13.8$ billion annually for direct medical treatment alone. The incidence of osteoporosis-related fractures is increasing and constitutes a major public health problem in the United States. Although several drug therapies are available for preventing osteoporotic fractures, the escalating drug costs bring about a heavy economic burden to consumers and third-party payers, and make it difficult for them to make decisions purely based on treatment effectiveness, especially for those expensive new drugs. The three 
drug therapies HRT, alendronate, and raloxifene evaluated in this study were the most competitive and commonly used preventive drug therapies for osteoporotic fractures at the current time. The study population was WV Medicaid women with age of 45 to 85 years. The goal of this study was to provide information to WV Medicaid decision makers regarding the relative cost effectiveness of these three preventive drug therapies based on the risk distribution of their population.

The study was conducted in three phases. Phase I identified the direct medical costs and major adverse events associated with each therapy. Treatment compliance patterns with the three drug therapies were also ascertained. Phase II estimated osteoporotic fracture risk distribution in the study population based on well-recognized risk factors. Phase III utilized the data from Phase I and Phase II to establish a decision model incorporating women's risk of osteoporotic fractures in the study population. The cost-effectiveness ratios by risk levels were calculated for each drug therapy.

\section{Discussion of Results}

\section{Cost-Effectiveness of the Three Drug Therapies}

Results from the first analysis using mean values of direct treatment costs, risk reduction rate, and background risk revealed that $\$ 12,401$ were needed for HRT to prevent a fracture, and $\$ 16,082$ and $\$ 18,685$ were needed for alendronate and raloxifene, respectively. In addition, compared to HRT, to prevent one more fracture, an extra cost of $\$ 23,799$ was needed for alendronate therapy. An extra cost of $\$ 37,782$ was needed for raloxifene therapy to prevent one more fracture than alendronate. Simple sensitivity 
analysis was conducted to identify the key variables and evaluate the robustness of the model. It showed that the $\mathrm{CE}$ ratios were sensitive to treatment costs and risk reduction rate, but not much sensitive to AE probability and willingness-to-continue rate. The preference for alendronate or raloxifene could be altered depending upon treatment costs and risk reduction rates. However, HRT always had lowest costs per fracture averted compared to the other two treatments in the sensitivity analysis, even though its CE was also sensitive to treatment costs and risk reduction rates. Therefore, HRT seemed to be the most cost effective therapy.

Since the above CEs were calculated based on the mean values of key variables, they did not reflect the variety of key variables and the uncertainty risk in data. Although simple sensitivity analysis can be helpful in identifying factors that significantly affect $\mathrm{CE}$ ratios and collecting information in a more efficient manner, single-value analysis and even scenario analysis can be extremely misleading (Torries, 1997). In reality, within the possible range of each variable, there are thousands of different value combinations exist. It is unlikely that a single variable changes while others keep constant (i.e., single value sensitivity analysis) or several variables change to certain values at the same time (i.e., scenario analysis). In this study, a probabilistic analysis using Monte Carlo simulation method was conducted to overcome the disadvantages of simple sensitivity analysis. The probabilistic CE analysis takes into account all possible situations/scenarios simultaneously. The study applied the distribution of each key variable. Values were randomly sampled from these distributions (input variables). In Data $^{\mathrm{TM}}$ 3.5, a distribution will be sampled only in Monte Carlo simulations. Since inputs 
were probabilistic, most of uncertainty or risk inherent in the intervention was reflected in the range of input variables. The result or output variable was also probabilistic and the expected value (median value) was used in the report to make comparisons.

Results from Monte Carlo simulation showed that $\$ 29,119$, instead of $\$ 12,401$ in the analysis using mean values, were expected for HRT to prevent a fracture, and $\$ 35,101$ (instead of $\$ 16,082$ ) and $\$ 39,760$ (instead of $\$ 18,685$ ) were expected for alendronate and raloxifene, respectively. Furthermore, compared to HRT, to prevent one more fracture, an extra cost of $\$ 42,181$ was needed for alendronate therapy. The extra cost for raloxifene to prevent one more fracture was more than doubled comparing to alendronate marginal costs and reached as high as $\$ 85,509$.

The expected values of the three treatment strategies from Monte Carlo simulation was much higher than that from the analyses using single mean values. This is because the uncertainty in data or the risk has been taken into account and reflected in the expected value. Additionally, in some project evaluations, risk preference theory is used to determine the actual value of an intervention or a project (certainty equivalence) based upon investors' risk attitudes. Theoretically, what the investor would eventually accept/pay should be lower (for costs) or higher (for benefits) than the expected value of an intervention from Monte Carlo simulation because the risk preferences of the investor have not yet been considered in the later.

Findings from both conventional $\mathrm{CE}$ analysis and probabilistic analysis revealed that HRT was the most cost-effective therapy to prevent osteoporotic fractures. The findings are consistent with the literature. Despite inadequate data and some conflicting 
findings on long-term, multi-system effects, most pharmacoeconomic studies concluded that HRT was both medically and economically beneficial in postmenopausal women, especially for those who have undergone hysterectomy and/or with high treatment compliance rate. For women with prior hysterectomy, estrogen therapy should always be the first choice, and the $\mathrm{CE}$ is better because of the absence of risk of endometrial cancer or uterine bleeding and the lack of need for biopsies (Willhite, 1998; Johannesson and Jönsson, 1993). Although there lacks formal data from pharmacoeconomic evaluations, most researchers believe that alendronate and raloxifene are unlikely to replace HRT in the prevention of osteoporosis and fractures and should be used as a second-line alternative for women intolerant to HRT or not willing to take HRT (Willhite, 1998; DeGregorio and Taras, 1998). No study has been done to compare the cost-effectiveness of alendronate and raloxifene. This is partly because their long-term effects are still not available.

Some people argue that ethically, treatments with higher effectiveness, regardless of their costs, should be favored. Although HRT is the cheapest therapy and have showed cost-effective in most studies (including this study), it does not mean that alendronate and raloxifene will be out of consideration. Even in a cost-sensitive managed care environment, the treatment with lowest CE ratio may not always be the first choice. The definition of a "cost effective therapy" should be based on the comparisons to the CE ratios of treatments for various diseases. Cost-effectiveness ratios (cost/life-year gained) for some commonly reimbursed medical interventions include $\$ 237,000$ for liver transplantation (1992 dollars), \$232,000 for screening mammography for women 
younger than age 50, $\$ 45,000$ (1995 dollars) for screening mammography for women older than age 50 , and $\$ 82,000$ to $\$ 88,500$ (1992 dollars) for zidovudine for human immunodeficiency virus infections (Noe et al., 1999). Although the threshold for determining whether an intervention is cost effective is hard to define and generalize, less than $\$ 50,000$ per life-year gained has been sited as acceptable for therapeutic interventions, and more than $\$ 100,000$ per life-year gained is generally considered excessive (Mark et al., 1995). Nevertheless, many interventions that cost as much as $\$ 100,000$ per year-of-life saved have been accepted (Hillman and Kim, 1995).

The available data do not allow direct comparisons of the CE ratios obtained in this study to those using life-year gained as effectiveness measures in the literature. Also, some studies use quality-adjusted life year (QALY) gained and some use non-quality adjusted life year gained (which is solely based on mortality rate). Even among studies using QALY, different utility values have been applied. If an average value of 0.1 lifeyear gained for one vertebral or hip fracture prevented, the expected CE ratios obtained in this study appear to be moderate in relation to the CE of breast cancer, hypertension and hypercholesterolemia prevention (Noe et al, 1999; Smith \& Hillner, 1993; Whittington \& Faulds, 1994).

It should be emphasized that the acceptable level of CE or incremental costs may vary greatly by different perspectives taken (e.g., patients' perspective $v s$. third party payers' perspective). The interpretation of satisfactory CE ratios highly depends on individual decision makers. The acceptability of treatments with high effectiveness and 
high costs such as alendronate and raloxifene in this study may also be favored in certain circumstances.

\section{Applying Background Risk Distribution to the Economic Assessment}

Cost-effectiveness of a preventive intervention is affected by the background risk of the disease and varies in different populations (Jönsson, 1998). To evaluate the influence of background fracture risk in the study population on the cost-effectiveness of each drug therapy, the background risk distribution was applied to the CE analysis. Furthermore, the background risk was stratified as three levels: low, medium, and high. $\mathrm{CE}$ ratios were calculated for women in each risk category.

Results showed that the average fracture risk was 0.22 in the study population. Using a risk level of 0.1 and 0.3 as cutoff values, $42.6 \%$ women in the study sample had less than $10 \%$ chance to develop osteoporotic fracture (low risk group); $31.5 \%$ women had less than $30 \%$ but greater than $10 \%$ chance to develop osteoporotic fracture (medium risk group); and $25.9 \%$ women had a fracture risk greater than $30 \%$ (high risk group). Since there is no information on categorizing risk levels based on risk distribution data in the literature, these cutoff values were arbitrary. However, in a report of WV Bureau for Public Health ("The Burden of Osteoporosis in West Virginia", 1999), the estimated 1996 osteoporosis rate in West Virginia women aged over 50 years was $21 \%$, the rate of women with low bone mass was $39 \%$. If we assume that the rate of women with high fracture risk could be reflected by the rate of osteoporosis, and women with low bone mass had medium fracture risk, the percentages of women in each risk category from this 
study are similar to those from the WV report. Thus the projected risk cutoff values may be reasonable to the study population.

To illustrate whether and how the cost-effectiveness of each drug therapy was affected by the background risk levels, CE ratios were calculated in women with different risk categories. Monte Carlo simulation procedure was conducted for each risk group. Results showed that the cost effectiveness ratios decreased dramatically with increased fracture risk for all three therapies. For HRT, the expected costs for preventing one fracture compared to no therapy were $\$ 79,130, \$ 19,569$, and $\$ 7,232$ for the low, medium, and high risk group, respectively. For alendronate and raloxifene therapy, although the expected costs for preventing one fracture were as high as $\$ 105,911$ and $\$ 140,675$ for low risk women - almost doubled than the corresponding HRT costs, the expected costs reduced dramatically to $\$ 9,690$ for alendronate and $\$ 11,536$ for raloxifene in high risk women. This indicates that with the increase of fracture risk, alendronate and raloxifene become more cost-effective choices and competitive with HRT.

A same trend was observed in the incremental CE ratios. Among low risk women, $\$ 151,981$ were estimated for alendronate to prevent one extra fracture compare to HRT, and $\$ 697,270$ were estimated for raloxifene to prevent one extra fracture than alendronate; while among high risk women, the marginal costs were down to \$11,099 and $\$ 34,017$ for alendronate and raloxifene, respectively. Therefore, background risk plays a critical role in determining cost-effectiveness and marginal costs of preventive treatments, especially for treatments with high costs and high benefits. Non-risk stratified approach in economic evaluations may considerably overestimate the costs of 
some more effective but expensive treatments, and provide a negative impression to decision-makers.

In the context of cost-effectiveness analysis, one alternative is defined to be dominated by another if the first both costs more and is less effective (Treeage software, Inc., 1999). The dominated alternative will usually be removed from consideration in decision making. In this study, no treatment was dominated by any of the other two treatments. Also, no "willingness-to-pay" of decision makers on minimum effectiveness and/or maximum costs were considered. Therefore, it is not an easy decision on which therapy should be taken or covered and which therapy should not. In such circumstances, applying background risk to the cost-effectiveness analysis and obtaining risk-stratified $\mathrm{CE}$ ratios would be more relevant and meaningful.

\section{Background Risk Evaluation}

As discussed above, the $\mathrm{CE}$ ratio of a preventive intervention is sensitive to changes in the background risk. Measuring background risk also helps to estimate the preventable fraction of the events (fracture), by obtaining the relative risk which is the comparison of risks among women with and without treatment. However, consensus on background fracture risk is difficult to achieve since fracture risk, in both general and osteoporotic populations, can vary widely because of measurement methods, and geographical variations. The major problem for estimating background fracture risk is to obtain a risk function that predicts fracture risk for a specific population. Many researchers have estimated fracture risk by predicting low bone mineral density (BMD) the single greatest risk factor of fracture (Ross et al., $1988^{1,2}$; Lindsay \& Cosman, 1990; 
Consensus Development Conference, 1993; Carroll et al., 1997). However, uncertainty still exists in estimating the association of BMD and other explanatory variables (factors) such as age, weight, and family history. Moreover, no quantitative relationship has been established between fracture risk and BMD level. The prediction model gets more complicated when different types of fracture and bone mineral density at different sites are considered.

In order to obtain a best estimation on the fracture risk among WV Medicaid postmenopausal women who had not experienced osteoporotic fracture and not received any preventive drugs, a risk assessment model was built for this specific population using those widely reported risk factors. The risk factors included in this study were age, age at menopause, current body mass index (BMI), family history, physical job, smoking, and alcohol consumption, while controlling for general health and some medications use. Race was not included in the model since more than $96 \%$ of WV population are white. For peak BMD, $25 \%$ data were missing (153 out of 772 women). Also, the current BMD and peak BMD were significantly correlated $(r=0.43, p<.001)$. Therefore, only current BMD was included in the model. Other risk factors such as exercise and intake of calcium/calcium supplement, vitamin D, and multivitamin pills were excluded from the final study model due to the lack of variety between case and control groups. Finally, there was significant difference in women's general health and the use of diuretics, steroid, and sedatives between two groups. Thus, these variables were included to control their effects on fracture risk. 
When general health and the use of some influential medications were controlled, family history was the most powerful determinant. The fracture risk of a woman with family history of osteoporosis and/or osteoporotic fracture was more than 8 times higher than that of a woman without such family history. Women with lower BMI score were also more likely to develop a fracture. In addition, the risk of fracture risk significantly associated with the age at menopause. The younger when a woman had her menopause, the greater risk she has to have an osteoporotic fracture. These findings are consistent with the literature (e.g., Carroll et al., 1997; Ribot et al., 1992; Turner et al., 1998). However, unlike many studies, this study did not detect a relationship between age and fracture risk. The lack of heterogeneity in age distributions between the two groups may be due to a sample selection bias. In the sample selection, to ensure enough cases were obtained in the younger group (women with age of 45-64), all fracture women had been included as cases, comparing to that in the 65-85 age group, only 500 women were randomly selected from all the women with fractures. Another explanation is that the effect of age on fracture risk was overwhelmed by that of other risk factors such as family history and BMI.

As reported in some studies (Reid et al., 2000; Sebastian, 2000; LaCroix et al., $2000)$, the use of diuretic medications were found to lower fracture risk by $56 \%(\mathrm{OR}=$ .44) in this study. Thiazide diuretics reduce urine calcium excretion and might therefore reduce postmenopausal bone loss. In some, but not all, case-control studies, their use has been associated with a reduced incidence of hip fractures (Reid et al., 2000). Since hypertension has high prevalence in aged people and thiazide diuretics are one of the 
most commonly prescribed medications for this health condition, the protective effect of diuretics on osteoporotic fracture should be well recognized. Alcohol use, smoking, and physical activity were found not significantly related to fracture risk in this study. The relationship between these risk factors and fracture risks is also indefinite in the literature. For example, alcohol use has been associated with low bone density and fractures in some studies due to poor nutrition, impaired calcium and vitamin D metabolism, and risk of falls (Moniz, 1994), but some studies did not find this relationship (Turner et al., 1998). Cigarette smoking is also found to decrease estrogen concentrations, lower body weight, and cause early menopause and thus increase the risk of fracture (Hollenbach et al., 1993). However, some studies did not find cigarette smoking related to a increased fracture risk in postmenopausal women (Franceschi et al., 1996; Turner et al., 1998). Vitamin D in combination with calcium increased bone density and decrease fracture rate in some studies (Dawson-Hughes et al., 1997; Willhite, 1998) but not in others (Lips et al., 1996; Turner et al., 1998; Franceschi et al., 1996), depending on the population studied and doses used. Finally, exercise such as running, walking, or weight lifting may maintain or increase bone density in combination with calcium and vitamin D (Reid, 1996; Cumming \& Nevitt, 1997). However, another study indicated that excessive exercise many increase bone loss and fracture risk (Cumming, 1996).

The risk assessment model $\left(-2 \mathrm{LL}=275.81, \mathrm{R}^{2}=0.48\right)$ had a low sensitivity $(44 \%)$ and high specificity (95\%). A hold-out sampling analysis for model validation provides a quick way to demonstrate a consistency or reliability of the sensitivity/specificity. The results indicated that $44 \%$ of the women with fracture could be predicted as positive by 
the model, and approximately $95 \%$ of the women without fracture could be predicted as negative by the model. Also, approximately $81 \%$ of the women with a positive prediction result actually had fracture (PPV) and $77 \%$ of the women with a negative prediction result actually did not have fracture (NPV). The False Positive Rate and False Negative Rate revealed that $19 \%$ of the women who predicted as positive but actually did not have fracture and $23 \%$ of the women who predicted as negative but actually had fracture. An overall accuracy rate of $78 \%$ was relatively high, meaning that $78 \%$ of the women tested could be classified as positive or negative correctly. A high $c$ statistic of 0.85 was also obtained for the study model, which means in almost $85 \%$ of all possible pairs of subjects in which one subject had fracture and the other did not, the projected model assigned a higher probability of having fracture to the subject with fracture.

The model had a relatively low sensitivity but very high specificity. Since the study goal was to develop a risk assessment model for women without fracture rather than a diagnostic tool, the high specificity and overall prediction accuracy may be more important. The low sensitivity of the model was unlikely to affect the estimation on risk distributions. Compared to the risk assessment models developed by other studies (e.g., Lydick et al., 1998; Carroll et al., 1997; Blalock et al., 1996; Franceschi et al., 1996; Elliot et al., 1993; Robit et al., 1992), the model established in this study had a relatively strong capability of predicting women's osteoporotic risk among disease free women. For example, a risk assessment model for postmenopausal osteoporosis using clinical factors showed a sensitivity of $73 \%$ and a specificity of $66 \%$. Elliot et al. (1993) used historical risk factors to predict low BMD. Their model produced a sensitivity of $86 \%$ 
and a low specificity of $25 \%$. Another low BMD prediction model also had a relatively high sensitivity ( $89 \%)$ but low specificity $(50 \%)$.

\section{Implications of the Study Results}

As stated in the previous chapter, the health care system nationwide has been concerned by escalating drug product costs. The introduction of new chemo-preventive agents with high effectiveness but also high costs may further increase drug treatment costs. Managed care providers or third-party payers have been challenged by the question of whether all preventive drug therapies with well-demonstrated efficacy should be covered. Due to limited health care resources, the decision should be based on both effectiveness and costs of a drug therapy. There is a great need to measure and compare value of pharmaceutical products/services. This study evaluated cost-effectiveness ratios of three comparable preventive drug therapies. The information could help decision makers understand the overall value or benefit of drug therapies and determine the most appropriate intervention(s) that should be covered to achieve their outcome goals.

Additionally, the study provides a framework to make risk-appropriate coverage decisions for competitive drug therapies. The study illustrated the relationship between varying risk levels and the range of economic and clinical impact. Few studies have been done to evaluate risk-stratified CE ratios of preventive interventions (Jönsson, 1998). Findings from this study revealed that targeting high-risk populations is very important to achieve optimal cost- effectiveness for interventions, especially for those with high cost and high effectiveness. This information could provide evidence on the need of a 
stratified preventive intervention approach. Previous studies have shown that women at the greatest risk of fracture are only slightly more likely to receive treatment that reduces fracture risk than women who are at low risk (Cummings, 1998). A non-stratified population-based prevention strategy may undertreat high-risk women and overtreat lowrisk women, and thus could not achieve an optimal cost-effectiveness or overall outcomes. The study illustrated significant variance in the cost-effectiveness ratios among women with different risk levels for all three drug therapies. This could increase the awareness and knowledge of payers or decision makers on the different economic scenarios for each therapy, and help them determine the most cost-effective therapy based on their specific target population.

Since the economic assessment model developed in this study is particularly based on WV Medicaid population, the economic impact of different coverage decisions on WV Medicaid program could be further evaluated. The possible costs for the WV Medicaid program to prevent one fracture could be estimated based on the therapy choices and related $\mathrm{CE}$ ratios, as well as an average total number of women recipients with age of 45 to 85 years in each risk category. It will also make it possible to compare cost-effectiveness of various combinations of the three therapies based on the fracture risk levels. These results may be instructive for WV Medicaid program policy makers in guideline constructing, budget planning, and coverage decision making.

Finally, the risk assessment model developed in this study could be used as a simple risk assessment tool. Clinicians could use it to quickly identify women with high or medium fracture risk as screening for more advanced test (e.g., BMD test) or more 
expensive drug therapy (e.g., instead of calcium supplement only). A valid and economical risk assessment tool is critical to achieve high effectiveness for a riskstratified prevention strategy. If the cost of detecting high-risk population is too high, the savings of a risk stratified approach over a mass approach will be offset by the high detection or screening costs. This will make it hard to achieve a cost-effectiveness goal, even for a well- targeted intervention. It should always be aware that the costs of evaluating women's fracture risk are included in the total intervention costs for a riskstratified intervention. However, the cost of a simple assessment based on some medical and lifestyle risk factors may be neglected. This study presented such simple selfreported risk assessment questionnaire and validated it in the study population.

In summary, although there are many uncertainties existing in the literature, and the results from the cost effectiveness analysis were sensitive toward different assumptions, the economic decision model developed in this study can still contribute many important insights to the evaluation of competitive drug therapies. This study provides a good way to structure the problems inherent in the methodology and identify the areas where more accurate information is needed. As new results and data become available, the model could be improved by incorporating them into the model.

\section{Recommendations for Future Research}

There are several major limitations of this study. First of all, the study only examined general number of fractures averted and did not explore the analysis by the 
type of fractures. Whenever the data becomes available for alendronate and raloxifene on risk reduction rates of different types of fractures, the economic evaluation model should be updated and CE analysis should be done for each major type of fractures. Future studies should also enhance the control for the non-osteoporotic causes of fractures.

In addition, the model is sensitive to the risk reduction rates. There is considerable uncertainty concerning the effects of preventive treatments, especially longterm and multi-system effects. This makes it difficult to simulate the effectiveness values precisely. Moreover, the relationship of risk reduction rates (or treatment effects) and treatment duration is also still unknown. Research is needed to evaluate the discontinuation effects for each studied drug therapy. Further studies should be conducted to obtain more accurate information based on updated findings from clinical trials and/or long-term epidemiology studies.

Unlike many pharmacoeconomic studies, quality adjusted life-year-gained were not used in this study as a measure of treatment effects. This makes a comparison difficult among diverse treatment strategies. However, there is little data on the effects of treatment strategies on health related quality-of-life (HRQL) (Taylor et al., 1997; Tosteson et al., 2000). Validated HRQL instruments are needed for proper evaluation. Appropriate assessment of utility is also critical to obtain a valid utility value, rather than an arbitrary value as used by many studies.

Patients' adherence is another important issue, especially for HRT and alendronate therapy. No treatment will be cost effective if patients do not take them as 
prescribed. Studies on exploring factors that affect patients' adherence behaviors are highly recommended, especially for alendronate and raloxifene therapy. As withdrawal and irregular bleeding are a well-recognized major reasons for non-adherence to HRT, (Scientific Advisory Board, 1996), the determinants for alendronate and raloxifene nonadherence need to be examined. The way to improve women's long-term medication adherence should also be stressed. There is little information on women's decision or preference when they are fully informed of risks, benefits, and alternative strategies. It will be interesting to find out how women's preference affects their adherence and even physicians' prescribing behaviors.

Finally, the risk assessment model developed in this study is only applied and validated in WV Medicaid population. The results obtained from this study must be interpreted with great care due to the assumptions used in the model. One of the important limitations in estimating the background risk is recall bias. However, if only women who had their first osteoporotic fracture in recent years (e.g., within the last 3) were included as cases in order to decrease the recall bias, many women with advanced age and/or having fracture at their early stage of menopause would be excluded. The sample would still be biased and unrepresentative, which makes it difficult to obtain the risk determinants and estimate fracture risk in the study population correctly. More studies need to be conducted to develop a valid and more generic risk assessment instrument. 


\section{Conclusions}

The study results suggest that the use of HRT to prevent osteoporostic fracture is most cost effective, even though it may have relatively high monitoring and AE costs, and low WTC rate. The greater number of fracture averted by alendronate and raloxifene were not sufficient to offset the higher treatment costs. It indicates that HRT may remain as the first-line therapy for preventing osteoporotic fracture. For women who are unwilling to take HRT or are contraindicated for HRT, alendronate or raloxifene will be another option. In addition, the cost-effectiveness ratios are influenced by the background risk. The significant decrease in marginal costs of alendronate and raloxifene in high-risk women indicates an economic condition to use these two drugs. Among women with high fracture risk, alendronate and raloxifene may be within the acceptable threshold of cost effectiveness for therapeutic interventions. 


\section{REFERENCES}

Abbott TA III, Manfredonis DJ, Stever GJ, Berger ML. A glimpse at the future burden of osteoporosis: the state fracture prediction model. West Point, PA: Outcomes Research and Management, Merck and Company, Inc. 1996.

ACCP (American College of Clinical Pharmacy). Pharmacoeconomics and outcomes: Applications for patient care. ACCP Publications. 1997.

Adachi JD, Bensen WG, Hodsman AB. Corticosteroid-induced osteoporosis. Seminars in Arthritis and Rheumatism. 1993; 22:375-384.

Adami S, Passeri M, Ortolani S, Broggini M, Carratelli L, Caruso I, et al. Effects of oral alendronate and intranasal salmon calcitonin on bone mass and biochemical markers of bone turnover in postmenopausal women with osteoporosis. Bone. 1995;17(4):383-90.

Adis International Limited. Postmenopausal osteoporosis: optimum time to start therapy unclear. Drug \& Ther Perspect. 1997; 10(7): 8-12.

Adis International Limited. Raloxifene: The first selective estrogen receptor modulator for postmenopausal osteoporosis. Drug \& Ther Perspect. 1998; 12(7): 1-5.

$\mathrm{Au} \mathrm{T}, \mathrm{Au}$ T. Chapter 3. Compound interest formulas and applications. In: Engineering economics for capital investment analysis. Eds, Au T \& Au T. 1992; 43-8.

Balfour JA, Goa KL. Roloxifene. Drug Aging. 1998; 12(4): 335-341.

Bauer DC, Frowner WS, Cauley JA, et al. Factors associated with appendicular bone mass in older women. Ann Intern Med. 1993; 118: 657-665.

Black DM, Cummings SR, Genant HK, et al. Axial and appendicular bone density predict fractures in older women. J Bone Min Res. 1992; 7: 633-638.

Black DM, Cummings SR, Karpf DB, et al. Randomized trial of effect of alendronate on risk of fracture in women with existing vertebral fractures. Lancet. 1996; 348(9041): $1535-41$.

Blalock SJ, DeVellis RF, Giorgino KB, et al. Osteoporosis prevention in premenopausal women using a stage model approach to examine the predictors of behavior. Health Psychol. 1996; 15(2): 84-93.

Bone HG, Downs RW Jr, Tucci JR, Harris ST, Weinstein RS, Licata AA, McClung MR, Kimmel DB, Gertz BJ, Hale E, Polvino WJ. Dose-response relationships for alendronate treatment in osteoporotic elderly women. Alendronate Elderly Osteoporosis Study Centers. J Clin Endocrinol Metab. 1997;82(1):265-74. 
Calcium Supplements. Med Let Drug Ther. 1989;31(805):101.

Carlson R. MORE not yet enough for raloxifene and breast cancer prevention. Inphama. $1998 ; 1141$.

Carroll J, Testa MA, Erat K, LeBoff MS, Fuleihan G. Modeling fracture risk using bone density, age, and years since menopause. Am J Prev Med. 1997;13(6):447-52.

Casagrande JT, Pike MC, Smith PG. An improved approximate formula for calculating sample sizes for comparing two binomial distributions. Biometrics. 1978;34:483-6.

Cauley JA, Seeley DG, Ensrud K et al. Estrogen replacement therapy and fractures in older women. Ann Intern Med. 1995;122:9-16.

Chesnut CH 3rd, McClung MR, Ensrud KE, Bell NH, Genant HK, Harris ST, Singer FR, Stock JL, Yood RA, Delmas PD, et al. Alendronate treatment of the postmenopausal osteoporotic woman: effect of multiple dosages on bone mass and bone remodeling. Am J Med. 1995;99(2):144-52.

Cheung AP, Wren BG. A cost-effectiveness analysis of hormone replacement therapy in the menopause. Med J Aust. 1992;156(5):312-6.

Chrischilles EA, Shireman T, Wallace R. Costs and health effects of osteoporotic fractures. Bone. 1994; 15: 377-386.

Clark AP, Schuttinga, JA. Targeted estrogen/progestogen replacement therapy for osteoporosis: Calculation of health care cost savings. Osteoporosis International. 1992;2: 195-200.

Colditz GA, Egan KM, Stampfer MJ. Hormone replacement therapy and risk of breast cancer: results from epidemiologic studies. Am J Obstet Gynecol. 1993;168(5):1473-80.

Collaborative Group on Hormonal Factors in Breast Cancer. Breast cancer and hormone replacement therapy: collaborative reanalysis of data from 51 epidemiological studies of 52,705 women with breast cancer and 108,411 women without breast cancer. Lancet. 1997;350:1047-59.

Compston JE. Prevention and management of osteoporosis: Current trends and future prospects. Drugs. 1997;53(5):727-735.

Consensus Development Conference. Diagnosis, prophylaxis, and treatment of osteoporosis. Am J Med. 1993;94:646-50.

Consensus Development Conference. Prophylaxis and treatment of osteoporosis. Am J Med. 1991; 90: 107-10. 
Cook DJ, Guyatt GH, Adachi JD, et al. Quality of life issues in women with vertebral fractures due to osteoporosis. Arthritis Rheum. 1993;36:750-756.

Cooper C. The crippling consequences of fractures and their impact on quality of life. $\mathrm{Am}$ J Med. 1997; 103 (Suppl 2A): 12S-19S.

Council of the National Osteoporosis Foundation. Guidelines for the early detection of osteoporosis and prediction of fracture risk. S Afr Med J. 1996;86(9):1113-6.

Cumming DC. Exercise induced amenorrhea, low bone density and estrogen replacement therapy. Arch Int Med. 1996;156:2193-5.

Cumming RG, Nevitt MC. Calcium for prevention of osteoporotic fractures in postmenopausal women. J bone Min Res. 1997;12:1321-9.

Cummings SR, Phillips SL, Wheat ME, et al. Recovery of function after hip fracture. $J$ Am Geriatr Soc. 1988; 36: 801-806.

Cummings SR, Rubin SM, Black D. The future of hip fractures in the United States: numbers, costs, and potential effects of postmenopausal estrogen. Clin Orthop. $1990^{1} ; 252: 163-6$.

Cummings SR, Browner WS, Grady D, Ettinger B. Should prescription of postmenopausal hormone therapy be based on the results of bone densitometry? Ann Intern Med. $1990^{2} ; 113(8): 565-7$.

Cummings SR, Black DM, Thompson DE, Applegate WB, Barrett-Connor E, Musliner TA, et al. Effect of alendronate on risk of fracture in women with low bone density but without vertebral fractures: results from the Fracture Intervention Trial. JAMA. 1998;280(24):2077-82.

Cummings SR. Prevention of hip fractures in older women: a population-based perspective. Osteoporos Int. 1998;8 Suppl 1:S8-12.

Daly E, Roche M, Barlow D, Gray A, McPherson K, Vessey M. HRT: an analysis of benefits, risks and costs. Br Med Bull. 1992;48(2):368-400.

Daly E, Vessey MP, Hawkins MM, Carson JL, Gough P, Marsh S. Risk of venous thromboembolism in users of hormone replacement therapy. Lancet. 1996;348(9033):977-80.

Dargent-Molina P, Favier F, Grandjean H, Baudoin C, Schott AM, Hausherr E, et al. Fall-related factors and risk of hip fracture: the EPIDOS prospective study. Lancet. 1996;348(9021):145-9. 
Dawson-Hughes B, Harris SS, et al. Effect of calcium and vitamin D supplementation on bone density in men and women 65 years of age or older. N Engl J Med. 1997;337:670-6.

de Valk-de Roo GW, Stehouwer CD, Meijer P, Mijatovic V, Kluft C, Kenemans P, et al. Both raloxifene and estrogen reduce major cardiovascular risk factors in healthy postmenopausal women: A 2-year, placebo-controlled study. Arterioscler Thromb Vasc Biol. 1999;19(12):2993-3000.

DeGregorio MW, Taras TL. Hormone replacement therapy and breast cancer: Revisting the issues. J Am Pharm Assoc. 1998;38:738-46.

Delmas PD, Bjarnason NH, Mitlak BH, Ravoux AC, Shah AS, Huster WJ, Draper M, Christiansen C. Effects of raloxifene on bone mineral density, serum cholesterol concentrations, and uterine endometrium in postmenopausal women. $N$ Engl J Med. 1997;337(23):1641-7.

Dosh RM, Fukushima SN, Lewis JE, Ross RL, Steinman LA. Hormone Replacement Therapy for Menopause. In: The Taking Charge of Menopause Workbook. 1997. New Harbinger Publications, Inc. http://my.webmd.com/content/dmk/dmk article 56492

Egol KA, Koval KJ, Zuckerman JD. Functional recovery following hip fracture in the elderly. J Orthopaedic Trauma. 1997;11:594-9.

Eiken P, Nielsen SP, Kolthoff N. Effects on bone mass after eight years of hormonal replacement therapy. Br J Obstet Gynaecol. 1997 Jun;104(6):702-7.

Eli Lilly and Company. Evista (raloxifene) Package Insert. 1997.

Elliot JR, Gilchrist NL, Wells JE, Ayling E, Turner J, Sainsbury R. Historical assessment of risk factors in screening for osteopenia in a normal Caucasian population. Aust $N Z J$ Med. 1993;23(5):458-62.

Ensrud KE, Lipschutz RC, Cauley JA, et al. Body size and hip fracture risk in older women: A prospective study. Am J Med. 1997; 103: 274-280.

Ettinger B, Genant HK, Cann CE. Long-term estrogen replacement therapy prevents bone loss and fractures. Ann Intern Med. 1985;102:319-24.

Ettinger B, Grady D. The waning effect of postmenopausal estrogen therapy on osteoporosis. N Engl J Med. 1993;329(16):1192-3.

Ettinger B, Pressman A, Schein J. Clinic visits and hospital admissions for care of acidrelated upper gastrointestinal disorders in women using alendronate for osteoporosis. $\mathrm{Am}$ J Manag Care. $1998^{1}$;4(10):1377-82. 
Ettinger B; Pressman A; Bradley C. Comparison of continuation of postmenopausal hormone replacement therapy: transdermal versus oral estrogen. Menopause. $1998^{2} ; 5(3): 152-6$.

Ettinger B, Black DM, Mitlak BH, Knickerbocker RK, Nickelsen T, Genant HK, et al. Reduction of vertebral fracture risk in postmenopausal women with osteoporosis treated with raloxifene: results from a 3-year randomized clinical trial. Multiple Outcomes of Raloxifene Evaluation (MORE) Investigators. JAMA. 1999;282(7):637-45.

Falkeborn M, Persson I, Terent A, Adami HO, Lithell H, Bergstrom R . Hormone replacement therapy and the risk of stroke. Follow-up of a population-based cohort in Sweden. Arch Intern Med. 1993;153(10):1201-9.

Felson DT, Zhang Y, Hannan MT, et al. The effect of postmenopausal estrogen therapy on bone density in elderly women. $N$ Engl J Med. 1993;329:1141-6.

Finucane FF, Madans JH, Bush TL, Wolf PH, Kleinman JC. Decreased risk of stroke among postmenopausal hormone users. Results from a national cohort. Arch Intern Med. 1993;153(1):73-9.

Fleiss JL. Statistical methods for rates and proportions. 2nd edition. New York: John Wiley \& Sons, Ltd. 1981.

Franceschi S, Schinella D; Bidoli E; Dal Maso L, La Vecchia C, Parazzini F, et al. The influence of body size, smoking, and diet on bone density in pre- and postmenopausal women. Epidemiology. 1996;7(4):411-4.

Fuch VR, Zeckhauser R. Valuing health: A “priceless" commodity. Am Econ Rev. 1987;77:263-8.

Gallagher JC. Pathophysiology of osteoporosis. Semin Nephrol. 1992; 12: 109-115.

Gold DT. The clinical impact of vertebral fractures: Quality of life in women with osteoporosis. Bone. 1996; 18(Suppl 3): 185S-189S.

Goldstein SR, Scheele WH, Rajagopalan SK, Wilkie JL, Walsh BW, Parsons AK. A 12month comparative study of raloxifene, estrogen, and placebo on the postmenopausal endometrium. Obstet Gynecol. 2000 Jan;95(1):95-103.

Grady D, Rubin SM, Petitti DB, Fox CS, Black D, Ettinger B, et al. Hormone therapy to prevent disease and prolong life in postmenopausal women. Ann Intern Med. 1992;117(12):1016-37.

Green SB. How many subjects does it take to do a regression analysis? Multivariate Behavioral Research. 1991;26:499-510. 
Grodstein F, Stampfer MJ, Manson JE, Colditz GA, Willett WC, Rosner B, Speizer FE, Hennekens $\mathrm{CH}$. Postmenopausal estrogen and progestin use and the risk of cardiovascular disease. $N$ Engl J Med. 1996;335(7):453-61.

Grodstein F, Stampfer MJ, Colditz GA, Willett WC, Manson JE, Joffe M, Rosner B, Fuchs C, Hankinson SE, Hunter DJ, Hennekens CH, Speizer FE. Postmenopausal hormone therapy and mortality. N Engl J Med. 1997;336(25):1769-75.

Gutthann PS, Rodriguez LAG, Castellsague J, Oliart DA Hormone replacement therapy and risk of venous thromboembolism: population based case-control study. BMJ. 1997;314(7083):796-800.

Haines C, Chung T, Chang A, Masarei J, Tomlinson B, Wong E. Effect of oral estradiol on $\mathrm{Lp}$ (a) and other lipoproteins in postmenopausal women. A randomized, double-blind, placebo-controlled, crossover study. Arch Intern Med. 1996;156(8):866-72.

Heidrich F, Thompson RS. Osteoporosis prevention: Strategies applicable for general population groups. J Fam Pract. 1987; 25(1): 33-39.

Henderson VW, Paganini-Hill A, Miller BL, Elble RJ, Reyes PF, Shoupe D, McCleary CA, Klein RA, Hake AM, Farlow MR. Estrogen for Alzheimer's disease in women: randomized, double-blind, placebo-controlled trial. Neurology. 2000;54(2):295-301.

Hillman AL, Kim MS. Economic decision making in healthcare: A standard approach to discounting health outcomes. Pharmacoeconomics. 1995;7:198-205.

Hobbs FB. $65+$ in the United States. Washington, DC: U.S. Deptment of Commerce, Economics and Statistics Administration, Bureau of the Census. 1996.

Hollenbach KA, Barrett-Connor E, Edelstein SL, et al. Cigarette smoking and bone mineral density in older men and women. Am J Public Health. 1993;83:1265-70.

Hormone Replacement Therapy Technical Bulletin. Washington DC. The American College of Obstetrics and Gynecology. 1992;166(4).

Hosking D, Chilvers CE, Christiansen C, Ravn P, Wasnich R, Ross P, et al. Prevention of bone loss with alendronate in postmenopausal women under 60 years of age. Early Postmenopausal Intervention Cohort Study Group. N Engl J Med. 1998;338(8):485-92.

Huang C, Ross PD, Wasnich RD. Vertebral fracture and other predictors of physical impairment and health care utilization. Arch Intern Med. 1996; 156: 2469-2475.

Hui SL, Slemenda CS, Johnston CC. Age and bone mass as predictors of fracture in a prospective study. J Clin Invest. 1988; 91: 1804-1809. 
Hulley S, Grady D, Bush T, Furberg C, Herrington D, Riggs B, Vittinghoff E. Randomized trial of estrogen plus progestin for secondary prevention of coronary heart disease in postmenopausal women. Heart and Estrogen/progestin Replacement Study (HERS) Research Group. JAMA. 1998;280(7):605-13.

Iqbal MM. Osteoporosis: Epidemiology, Diagnosis, and Treatment. South Med J. 2000;93(1):2-18.

Isenbarger DW, Chapin BL Osteoporosis. Current pharmacologic options for prevention and treatment. Postgrad Med. 1997;101(1):129-32, 136-7, 141-2.

Jalovaara P, Virkkunen H. Quality of life after hemiarthroplasty for femoral neck fracture. Acta Orthop Scand. 1991; 62: 208-217.

Johannesson M, Jönsson B. Economic evaluation of osteoporosis prevention. Health Policy. 1993;24(2):103-24.

Johnell O, Gullberg B, Kanis JA, et al. Risk factors for hip fracture in European women: The MEDOS study. J Bone Miner Res. 1995; 10: 1802-1815.

Jones G, Nguyen T, Sambrook PN, et al. Symptomatic fracture incidence in elderly men and women: The Dubbo Osteoporosis epidemiology Study (DOES). Osteoporosis Int. 1994; 4: 277-282.

Jönsson B. Targeting high-risk populations. Osteoporos Int. 1998; (Suppl.1): S13-S16.

Kanis JA. Treatment of osteoporotic fracture. Lancet. 1984;1(8367):27-33.

Kanis JA, Geusens P, Christiansen C. Guidelines for clinical trials in osteoporosis: A position paper of the European Foundation for Osteoporosis and Bone Disease. Osteoporosis Int. 1991; 1: 182-188.

Kanis JA, Pitt FA. Epidemiology of Osteoporosis. Bone. 1992;13(Suppl 1): S7-S15.

Kanis JA, Melton LJ III, Christiansen C, Johnston CC, Khaltaev N. The diagnosis of osteoporosis. J Bone Min Res. 1994; 9: 1137-1141.

Kanis JA, Delmas P, Burckhardt P, et al. On Behalf of the European Foundation for Osteoporosis and Bone Disease. Guidelines for diagnosis and management of osteoporosis. Osteoporosis Int. 1997; 7:390-406.

Karpf DB, Shapiro DR, Seeman E, et al. Prevention of nonvertebral fractures by alendronate: a meta-analysis. JAMA. 1997; 277: 1159-64.

Keil DP, Felson DT, Anderson JJ, et al. Hip fracture and the use of estrogens in postmenopausal women. $N$ Engl J Med. 1987;317:1169-74. 
Koh KK, Cardillo C, Bui MN, Hathaway L, Csako G, Waclawiw MA, Panza JA, Cannon RO. Vascular effects of estrogen and cholesterol-lowering therapies in hypercholesterolemic postmenopausal women. Circulation. 1999;99(3):354-60.

Kroger H, Huopio J, Honkanen R, et al. Prediction of fracture risk using axial bone mineral density in a perimenopausal population: a prospective study. J Bone Min Res. 1995; 10: 302-306.

Kroger H, Tuppurainen M, Honkanen R, Alhava E, Saarikoski S. Bone mineral density and risk factors for osteoporosis--a population-based study of 1600 perimenopausal women. Calcif Tissue Int. 1994;55(1):1-7.

Kushner PR. A practical approach to managing osteoporosis. Hospital Medicine. 1998;34(6):15-16,19-20,23-25.

LaCroix AZ; Ott SM; Ichikawa L; Scholes D; Barlow WE. Low-dose hydrochlorothiazide and preservation of bone mineral density in older adults. A randomized, double-blind, placebo-controlled trial. Ann Intern Med. 2000;133(7):516-26.

Lauritzen JB, Schwarz P, Lund B, et al. Changing incidence and residual lifetime risk of common osteoporosis-related fractures. Osteoporosis Int. 1993; 3: 127-132.

Levin TR. Fosamax linked to greater need for GI symptom treatment. Presented at the American Society for Bone and Mineral Research (ASBMR) annual meeting. 1999,Oct.

Liberman UA, Weiss SR, Broll J, Minne HW, Quan H, Bell NH, Rodriguez-Portales J, Downs RW Jr, Dequeker J, Favus M. Effect of oral alendronate on bone mineral density and the incidence of fractures in postmenopausal osteoporosis. The Alendronate Phase III Osteoporosis Treatment Study Group. N Engl J Med. 1995;333(22):1437-43.

Licht, MH. Multiple regression and correlation in Grimm, LG, Yarnold, PR, (eds.): Reading and Understanding Multivariate Statistics. Washington, DC: American Psychological Association; 1994.

Lilly Research Laboratories, Eli Lilly and Company. Adverse events reported by postmenopausal women in controlled trials with raloxifene. 1999;93(4):558-65.

Lindsay R, Cosman F. Epidemiology of osteoporosis. In: HRT and Osteoporosis. Chapter 7. Eds: Drife JO, Studd JWW. 1990. Springer-Verlag.

Lindsay R. The menopause: sex, steroids, and osteoporosis. Clin Obstet Gynecol. 1987;30:847-59.

Lips P, Graafmans WC, Ooms ME, et al. Vitamin D supplementation and fracture incidence in elderly persons: a randomized, placebo-controlled clinical trial. Ann Intern Med. 1996;124:400-6. 
Lips P. Epidemiology and predictors of fractures associated with osteoporosis. Am J Med. 1997;103(2A):3S-8S.

Lipscomb J, Weinstein MC, Torrance GW. Time preference. In: Gold MR, Siegel JE, Russell LB, Weinstein MC, eds. Cost-effectiveness in Health and Medicine. New York, NY: Oxford University Press. 1996;214-46.

Lufkin EG, Wahner HW, O'Fallon WM, Hodgson SF, Kotowicz MA, Lane AW, Judd HL, Caplan RH, Riggs BL. Treatment of postmenopausal osteoporosis with transdermal estrogen. Ann Intern Med. 1992;117(1):1-9

Lydick E, Cook C, Turpin J, et al. Development and Validation of a simple questionnaire to facilitate identification of women likely to have low bone density. Am J Managed Care. 1998; 4: 37-48.

Magaziner J Simonsick EM, Kashner TM, et al. Predictors of functional recovery one year following hospital discharge for hip fracture: A prospective study. J Gerontol Biol Sci Med Sci. 1990; 45: M101-M107.

Margolis KL, Ensrud KE, Schreiner PJ, Tabor HK. Body size and risk for clinical fractures in older women. Study of Osteoporotic Fractures Research Group. Ann Intern Med. 2000;133(2):123-7.

Mark DB, Hlatky MA, Califf RM, et al. Cost effectiveness of thrombolytic therapy with tissue plasminogen activator as compared with streptokinase for acute myocardial infarction. N Engl J Med. 1995;332:1418-24.

Marotolli RA, Berkman LF, Cooney LM. Decline in physical function following hip fracture. J Am Geriatr Soc. 1992; 40: 861-866.

Melton LJ III, Kan SH, Wahner HW, Riggs BL. Lifetime fracture risk: an approach to hip fracture risk assessment based on bone mineral density and age. J Clin Epidemiol. 1988;41(10):985-94.

Melton LJ III, Atkinson EJ, O'Fallon WM, et al. Long-term fracture prediction by bone mineral assessed at different skeletal sites. J Bone Miner Res. 1993; 8: 1227-1233.

Melton LJ III. Epidemiology of age-related fractures. In: Avioli LV, ed. The Osteoporotic Syndrome. Detection, Prevention, and Treatment. $3^{\text {rd }}$ ed. New York: Wiley-Liss. 1993; 17-38.

Melton LJ III. Hip fractures: a worldwide problem today and tomorrow. Bone. 1993; 14 (Suppl): S1-S8.

Melton LJ III. How many women have osteoporosis now? J Bone Miner Res. 1995; 10: 175-177. 
Merck \& Co., Inc. Fosamax ${ }^{\circledR}$ Package Insert. 1995.

Meunier PJ, Delmas PD, Eastell R, et al. Diagnosis and management of osteoporosis in postmenopausal women: clinical guidelines. Clin Ther. 1999; 21(6):1025-1044.

Michaelsson K, Bergstrom R, Mallmin H, Holmberg L, Wolk A, Ljunghall S. Screening for osteopenia and osteoporosis: selection by body composition. Osteoporos Int. 1996;6(2):120-6.

Michaelsson K, Baron JA, Farahmand BY, Johnell O, Magnusson C, Persson PG, et al. Hormone replacement therapy and risk of hip fracture: population based case-control study. The Swedish Hip Fracture Study Group. BMJ. 1998;316(7148):1858-63.

Miller KL. Hormone replacement therapy in the elderly. Clin Obstet Gynecol. 1996;39:912-32.

Mizunuma H, Okano H, Soda M, Kagami I, Miyamoto S, Tokizawa T, Honjo S, Ibuki Y. Prevention of postmenopausal bone loss with minimal uterine bleeding using low dose continuous estrogen/progestin therapy: a 2-year prospective study. Maturitas. 1997;27(1):69-76.

Moniz C. Alcohol and bone. Br Med Bult. 1994;50:67-75.

Mosca L, Manson JE, Sutherland SE, Langer RD, Manolio T, Barrett-Connor E. Cardiovascular disease in women. A statement for healthcare professionals from the American Heart Association. Circulation. 1997;96:2468-82.

Mossey JM, Mutran E, Knott K, et al. Determinants of recovery 12 months after hip fracture: The importance of psychosocial factors. Am J Public Health. 1989; 79: 279-285.

Nabulsi AA, Folsom AR, White A, Patsch W, et al. Association of hormone-replacement therapy with various cardiovascular risk factors in postmenopausal women. The Atherosclerosis Risk in Communities Study Investigators. $N$ Engl J Med. 1993;328(15):1069-75.

National Institute of Arthritis and Musculoskeletal and Skin Diseases. Washington, DC: National Institutes of Health. National Resource Center for Osteoporosis and Related Bone Diseases, 1997. Internet page at URL: http:/www.nih.gov/niams/.

National Osteoporosis Foundation. 1996 and 2015 osteoporosis prevalence figures: stateby-state report. Washington DC. National Osteoporosis Foundation. Washington DC. 1997.

National Pharmaceutical Council. Pharmaceutical Benefits Under State Medical Assistance Programs. National Pharmaceutical Council, Reston, VA. 1983. 
National Pharmaceutical Council. Pharmaceutical Benefits Under State Medical Assistance Programs. National Pharmaceutical Council, Reston, VA. 1999.

National Pharmaceutical Council. Pharmaceutical Benefits Under State Medical Assistance Programs. National Pharmaceutical Council, Reston, VA. 2000.

Nevitt MC, Ettinger B, Black DM, et al. The association of radiographically detected vertebral fractures with back pain and function: A prospective study. Ann Intern Med. 1998; 128: 793-800.

Noe LL, Becker RV, Gradishar WJ, Gore M, Trotter JP. The cost effectiveness of tamoxifen in the prevention of breast cancer. Am J Manag Care. 1999;5(suppl):S389406.

Odell WD, Heath H III. Osteoporosis: pathophysiology, prevention, diagnosis, and treatment. Disease a Month. 1993; 39: 794-867.

Ongphiphadhanakul B, Rajatanavin R, Chailurkit L, Piaseu N, Teerarungsikul K, Sirisriro R, Komindr S, Pauvilai G/ Prediction of low bone mineral density in postmenopausal women by artificial neural network model compared to logistic regression model. J Med Assoc Thai. 1997;80(8):508-15.

Osteoporosis Task Force. AACE clinical practice guidelines for the prevention and treatment of postmenopausal osteoporosis. J Florida Med Assoc. 1996; 83:552-66.

Paganini-Hill A, Henderson VW. Estrogen replacement therapy and risk of Alzheimer disease. Arch Intern Med. 1996;156(19):2213-7.

Paganini-Hill A. Does estrogen replacement therapy protect against Alzheimer's disease? Osteoporos Int. 1997;7 Suppl 1:S12-7.

Paganini-Hill A. Oestrogen replacement therapy and Alzheimer's disease. Br J Obstet Gynaecol. 1996;103 Suppl 13:80-6.

Physicians' Desk Reference. 53 ${ }^{\text {rd }}$ ed. Raloxifene (Evista). Medical Economics Company, Inc. Montvale, NJ. 1999:1576-9.

Prestwood KM, Gunness M, Muchmore DB, Lu Y, Wong M, Raisz LG. A comparison of the effects of raloxifene and estrogen on bone in postmenopausal women. J Clin Endocrinol Metab. 2000;85(6):2197-202.

Prestwood KM, Weksler ME. Osteoporosis: up-to-date strategies for prevention and treatment. Geriatrics. 1997;52:92-4,97-8. 
Procter \& Gamble Pharmaceuticals. Didronel post-marketing surveillance study: the effects of cyclical etidronate treatment in actual clinical practice. Cincinnati, $\mathrm{OH}$. Final report, Study No: ED/41. 1996.

Ravnikar VA. Compliance with hormone therapy. Am J Obstet Gynecol. 1987; 156(5): 1332-1334.

Ray GT, Collin F, Lieu T, Fireman B, Colby CJ, Quesenberry CP, et al. The cost of health conditions in a health maintenance organization. Medical Care Research and Review. 2000;57(1):92-109.

Ray NF, Chan JK, Thamer M, Melton LJ III. Medical expenditures for the treatment of osteoporotic fractures in the United States in 1995. J Bone Miner Res. 1996;12:24-35.

Ray NF, Chan JK, Thamer M, Melton LJ III. Medical expenditures for the treatment of osteoporotic fractures in the United States in 1995: report from the National Osteoporosis Foundation. J Bone Miner Res. 1997;12(1):24-35.

Robertson J, Henry DA, O'Connell D, Lau E. Estrogens in the prevention of postmenopausal osteoporosis and fracture: a meta-analysis. Abstract no.072. PostMarketing Surveillance 7 (Special Issue): 1993;247.

Reid IR. Glucocorticoid-induced osteoporosis and other forms of secondary osteoporosis. In: Meunier PJ ed. Osteoporosis: Diagnosis and Management. London: Martin Dunitz. 1998; 233-250.

Reid IR, Ames RW, Orr-Walker BJ, Clearwater JM, Horne AM, Evans MC, et al. Hydrochlorothiazide reduces loss of cortical bone in normal postmenopausal women: a randomized controlled trial. Am J Med. 2000;109(5):362-70.

Reid JR. Therapy of osteoporosis: calcium, vitamin D, and exercise. Am J Med Sci. 1996;312:278-86.

Ribot C; Pouilles JM; Bonneu M; Tremollieres F. Assessment of the risk of postmenopausal osteoporosis using clinical factors. Clin Endocrinol (Oxf). 1992;36(3):2258 .

Riggs BL, Melton LJ III. The worldwide problem of osteoporosis: insights afforded by epidemiology. Bone. 1995; 17(suppl 5): 505S-511S.

Riggs BL, Melton LJ. Involutional Osteoporosis. N Engl J Med. 1986; 314: 1676-1686.

Robertson J, Henry DA, O'Connell D, Lau E. Estrogens in the prevention of postmenopausal osteoporosis and fracture: a meta-analysis. Abstract no.072. PostMarketing Surveillance 7 (Special Issue). 1993:247. 
Roche M, Vessey M. Hormone replacement therapy in the menopause: risks, benefits and costs. In: Drife JO \& Studd JWW (Eds). HRT and osteoporosis. Springer-Verlag London Ld. London. 1990;363-72.

Rosner AJ, Grima DT, Torrance GW, et al. Cost effectiveness of multi-therapy treatment strategies in the prevention of vertebral fractures in postmenopausal women with osteoporosis. Pharmacoeconomics. 1998; 14(5): 559-73.

Ross PD, Wasnich RD, Maclean CJ, et al. A Model for estimating the potential costs and savings of osteoporosis prevention strategies. Bone. 1988' ; 9: 337-347.

Ross PD, Wasnich RD, Vogel JM. Detection of prefracture spinal osteoporosis using bone mineral absorptiometry. J Bone Min Res. $1988^{2}$; 3: 1-11.

Ross PD, Davis JW, Vogel JM, Wasnich RD. A critical review of bone mass and the risk of fractures in osteoporosis. Calcif Tissue Int. 1990;46(3):149-61.

Ross PD, Davis JW, Epstein RS, et al. Pre-existing fractures and bone mass predict vertebral fracture incidence in women. Ann Intern Med. 1991; 114: 919-923.

Ross PD, Genant HK, Davis JW, et al. Predicting vertebral fracture incidence from prevalent fractures and bone density among non-black, osteoporotic women. Osteoporosis Int. 1993; 3: 120-127.

Ross PD. Osteoporosis: frequency, consequences, and risk factors. Arch Intern Med. 1996; 156: 1399-1411.

Ross PD. Clinical consequences of vertebral fractures. Am J Med. 1997;103(Suppl 2A): 30S-43S.

Ross PD. Predicting bone loss and fracture risk with biochemical markers: A review. $J$ Clin Densitom. 1999 Fall;2(3):285-94. Review.

Ross PD. Prediction of fracture risk. II: Other risk factors. Am J Med Sci. 1996; 312(6):260-9.

Roy JA, Sawka CA, Pritchard KI . Hormone replacement therapy in women with breast cancer. Do the risks outweigh the benefits? J Clin Oncol. 1996;14(3):997-1006.

Sagraves R, O’Connell MB, Carson DS, Mahoney JM. APhA special report: Therapeutic options for osteoporosis. Washington DC, American Pharmaceutical Association, 1993: $1-20$.

Salamone LM, Pressman AR, Seeley DG, et al. Estrogen replacement therapy: a survey of older women's attitudes. Arch Intern Med. 1996;156:1293-7. 
Schlesselman JJ. Case-control studies: Design, conduct, analysis. New York: Oxford University Press. 1982.

Schneider DL, Barrett-Connor EL, Morton DJ. Timing of postmenopausal estrogen for optimal bone mineral density. The Rancho Bernardo Study. JAMA. 1997;277(7):543-7.

Schurch MA, Rizzoli R, Mermillod B, et al. A prospective study on socioeconomic aspects of fracture of the proximal femur. J Bone Miner Res. 1996; 11: 1936-1942.

Scientific Advisory Board, Osteoporosis Society of Canada. Clinical practice guidelines for the diagnosis and management of osteoporosis. Can Med Assoc J. 1996;155(8):111329.

Sebastian A. Thiazides and bone. Am J Med. 2000;109(5):429-30.

Seeman E, Tsalamandris C, Bass S, Pearce G. Present and future of osteoporosis therapy. Bone. 1995;17(2 Suppl):23S-29S.

Sillero-Arenas M, Delgado-Rodriguez M, Rodigues-Canteras R, Bueno-Cavanillas A, Galvez-Vargas R. Menopausal hormone replacement therapy and breast cancer: a metaanalysis. Obstet Gynecol. 1992;79(2):286-94.

Smith TJ, Hillner BE. The efficacy and cost-effectiveness of adjuvant therapy of early breast cancer in premenopausal women. J Clin Oncol. 1993;11:771-6.

Society of Obstetricians and Gynecologists of Canada. Canadian Menopause Consensus Conference. J Soc Obstet Gynaecol Can.1994; 16(5):1-40.

SPSS Inc. SPSS 10.0 Software Manual. Logistic Regression. 1999.

Stock JL, Bell NH, Chesnut CH 3rd, Ensrud KE, Genant HK, Harris ST, McClung MR, Singer FR, Yood RA, Pryor-Tillotson S, Wei L, Santora AC $2^{\text {nd }}$. Increments in bone mineral density of the lumbar spine and hip and suppression of bone turnover are maintained after discontinuation of alendronate in postmenopausal women. Am J Med. 1997;103(4):291-7.

Strom BL. Chapter 3. Sample size considerations for pharmacoepidemiology studies. In: Pharmacoepidemiology. 3rd edition. New York: John Wiley \& Sons, Ltd. 2000.

Suarez-Almazor M, Homik JE, Messina D, Davis P. Attitudes and beliefs of family physicians and gynecologists in relation to the prevention and treatment of osteoporosis. J Bone Miner Res. 1997;12(7):1100-7.

The Writing Group for the PEPI Trial. Effects of estrogen or estrogen/progestin regimens on heart disease risk factors in postmenopausal women. JAMA. 1995;273:199-208. 
The Writing Group for the PEPI Trial. Effects of hormone therapy on bone mineral density: results from the Postmenopausal Estrogen/Progestin Interventions Trial. JAMA. 1996;276(17):1389-96.

Tosteson AN, Rosenthal DI, Melton LJ 3d, Weinstein MC. Cost effectiveness of screening perimenopausal white women for osteoporosis: bone densitometry and hormone replacement therapy. Ann Intern Med. 1990;113(8):594-603.

Tosteson AN, Weinstein MC. Cost-effectiveness of hormone replacement therapy after the menopause. Baillieres Clin Obstet Gynaecol. 1991;5(4):943-59.

Tosteson Anna. Hormone replacement therapy: Benefit, risk and cost considerations. $J$ Clin Pharmacol. 1994; 34: 719-722.

Torgerson DJ, Campbell MK, Thomas RE, Reid DM. Prediction of perimenopausal fractures by bone mineral density and other risk factors. $J$ Bone Miner Res. 1996;11(2):293-7.

Tosteson AN, Gabriel SE, Kneeland TS, Moncur MM, Manganiello PD, Schiff I, Ettinger B, Melton LJ 3rd. Has the impact of hormone replacement therapy on health-related quality of life been undervalued? J Womens Health Gend Based Med. 2000;9(2):119-30.

Treeage software, Inc. DATATM 3.5 for healthcare users' manual. Chapter 21: Costeffectiveness analysis. 1999.

Truter I, Serfontein JH. A survey of the treatment of female patients with osteoporosis using drug utilization consumption parameters. J Clin Pharm Ther. 1999;24(3):209-17.

Tucci JR, Tonino RP, Emkey RD, Peverly CA, Kher U, Santora AC $2^{\text {nd }}$. Effect of three years of oral alendronate treatment in postmenopausal women with osteoporosis. Am J Med. 1996;101(5):488-501.

Turner LW; Fu Q; Taylor JE; Wang MQ. Osteoporotic fracture among older U.S. women: risk factors quantified. J Aging Health. 1998;10(3):372-91.

Ullom-Minnich P. Prevention of osteoporosis and fractures. Am Fam Physician. 1999;60(1):194-202.

Umland EM, Rinaldi C, Parks SM, Boyce EG. The impact of estrogen replacement therapy and raloxifene on osteoporosis, cardiovascular disease, and gynecologic cancers. Ann Pharmacother. 1999;33:1315-28.

van der Voort DJ, Brandon S, Dinant GJ, van Wersch JW. Screening for osteoporosis using easily obtainable biometrical data: diagnostic accuracy of measured, self-reported and recalled BMI, and related costs of bone mineral density measurements. Osteoporos Int. 2000;11(3):233-9. 
van Leeuwen FE, Benraadt J, Coebergh JWW, et al. Risk of endometrial cancer after tamoxifen treatment of breast cancer. Lancet. 1994; 343: 448-452.

Walsh BW, Kuller LH, Wild RA, Paul S, Farmer M, Lawrence JB, et al. Effects of raloxifene on serum lipids and coagulation factors in healthy postmenopausal women. JAMA. 1998;279(18):1445-51.

Walsh BW, Paul S, Wild RA, Dean RA, Tracy RP, Cox DA, Anderson PW The effects of hormone replacement therapy and raloxifene on C-reactive protein and homocysteine in healthy postmenopausal women: a randomized, controlled trial. J Clin Endocrinol Metab. 2000;85(1):214-8.

Weinstein MC, Schiff I. Cost-effectiveness of hormone replacement therapy in the menopause. Obstet Gynecol Surv. 1983;38(8):445-55.

Weinstein MC, Tosteson ANA. Cost-effectiveness of hormone replacement. Annals of the New York Academy of Sciences. 1990;592:162-92.

Weinstein MC. Estrogen use in menopausal women - costs, risks, and benefits. NEJM. 1980;303:308-16.

Weiss NS, Ure CL, Ballard JH, et al. Decreased risk of fractures of the hip and lower forearm with postmenopausal use of estrogen. NEJM. 1980; 303: 1195-1198.

Whittington R, Faulds D. Hormone replacement therapy II. A pharmacoeconomic appraisal of its role in the prevention of postmenopausal osteoporosis and ischaemic heart disease. Pharmacoeconomics. 1994; 5(6): 513-554.

Wild RA. Estrogen: effects on the cardiovascular tree. Obstet Gynecol. 1996;87:27S-35S.

Willhite L. Osteoporosis in Women: Prevention and treatment. J Am Pharm Assoc. 1998; 38: 614-624.

Wright RE. Reading and understanding multivariate statistics. Chapter 7. Logistic regression. In: Grimm LG, Yarnold PR (Eds). American psychological association. Washington DC. 1995.

WV Bureau for Public Health. The burden of osteoporosis in West Virginia. Department of Health and Human Resources, Bureau for Public Health, Office of Epidemiology and Health Promotion. 1998.

Wysowski DK, Golden L, Burke L. use of menopausal estrogens and medroxyprogesterone in the United States, 1982-1992. Obstet Gynecol. 1995;85(1):6-10.

Zumoff B. Biological and endocrinological insights into the possible breast cancer risk from menopausal estrogen replacement therapy. Steroids. 1993;58(5):196-204. 


\title{
Appendix A.
}

\author{
Average Wholesale Prices \\ Used by WV Medicaid Program (1999)
}




\begin{tabular}{|c|c|c|}
\hline Generics & Selected Brand Names & Unit Price (\$) \\
\hline $\begin{array}{l}\text { Conjugated } \\
\text { Estrogen } \\
0.625 \mathrm{mg} \\
\text { Package: } 30\end{array}$ & $\begin{array}{l}\text { Premarin } \\
\text { Ortho-Est } \\
\text { Ogen } \\
\text { Menest } \\
\text { Estratab }\end{array}$ & $\begin{array}{l}0.53 \\
0.46 \\
0.68 \\
0.60 \\
0.48\end{array}$ \\
\hline $\begin{array}{l}\text { Medroxyprogesterone } \\
5 \mathrm{mg}, 2.5 \mathrm{mg} \\
\text { Package: } 30\end{array}$ & $\begin{array}{l}\text { Provera }(5 \mathrm{mg}) \\
\text { Provera }(2.5 \mathrm{mg})\end{array}$ & $\begin{array}{l}0.71 \\
0.51\end{array}$ \\
\hline $\begin{array}{l}\text { Transdermal Estradiol } \\
\text { 0.37 mg or } 0.38 \mathrm{mg} \\
\text { Package: } 4\end{array}$ & Climara & 6.58 \\
\hline $\begin{array}{l}\text { Alendronate } \\
5 \mathrm{mg} \\
\text { Package: } 30\end{array}$ & Fosamax & 1.95 \\
\hline $\begin{array}{l}\text { Raloxifene } \\
60 \mathrm{mg} \\
\text { Package: } 30\end{array}$ & Evista & 2.03 \\
\hline
\end{tabular}




\section{Appendix B.}

\section{List of CPT Codes Used in the Study}




\section{BMD Test}

76075 Dual energy x-ray absorptiometry (DEXA), bone density study, one or more sites; axial skeleton (e.g., hips, pelvis, spine).

76076 Appendicular skeleton (peripheral) (e.g., radius, wrist, heel).

\section{Endometrial biopsy}

56351 Hysteroscopy, surgical; with sampling (biopsy) of endometrium and/or polypectomy, with or without D \& C.

58100 Endometrial sampling (biopsy) with or without endocervical sampling (biopsy), without cervical dilation any method (separate procedure).

\section{Intravascular Ultrasound}

93965 Diagnostic ultrasound (non-invasive)

\section{Mammography}

76092 Screening mammography, bilateral

\section{Physician Office Visits}

99271-99275 Office Consultations

99211-99215 Established patients

99201-99205 New patients

\section{Vaginal Ultrasound}

76830 Echography, transvaginal

Source: American Medical Association. Current Procedural Terminology $\left(c p t^{\mathrm{TM}}\right) .1999$. 


\section{Appendix C.}

\section{Expert Panel Survey}




\section{EXPERT PANEL SURVEY}

\section{On The Use Of HRT, Alendronate (Fosamax $®$ ), And Raloxifene (Evista $\left.{ }^{\circledR}\right)$ \\ In Preventing Osteoporotic Fractures Among Postmenopausal Women}

\section{Question I. Drug Dosage/Formulation and Patient Distribution (\%)}

Based on your practice, please estimate the proportion of patients on each therapy formulation for PREVENTING FRACTURES in women with or without diagnosed osteoporosis.

\section{HRT}

Cyclic estrogen $0.625 \mathrm{mg} /$ day + cyclic medroxyprogesterone $5 \mathrm{mg} /$ day $\%$

Continuous estrogen $0.625 \mathrm{mg} / \mathrm{day}+$ continuous medroxyprogesterone $2.5 \mathrm{mg} / \mathrm{day}$ $\%$

Cyclic transdermal estradiol 50mg/day + cyclic medroxyprogesterone 5mg/day $\%$

Continuous transdermal estradiol 50mg/d + continuous medroxyprogesterone $2.5 \mathrm{mg} / \mathrm{d}$ $\%$

\section{$>$ Alendronate}

$10 \mathrm{mg} /$ day $\%$

$5 \mathrm{mg} /$ day $\%$

$>$ Raloxifene

$60 \mathrm{mg} /$ day $\%$

$30 \mathrm{mg} /$ day $\%$

\section{Question II. Adverse Events \& Costs}

\section{$>$ HRT}

Major AEs

Vaginal bleeding

GI complaints

Leg pain/DVT

Other

\author{
Frequency (\%) Treatment Needed
}

$\square$ Yes $\square$ No

$\square$ Yes $\square$ No

$\square$ Yes $\square$ No

$\square$ Yes $\square$ No

$\begin{array}{lcc}- & \square \text { Yes } & \square \text { No } \\ - & \square \text { Yes } & \square \text { No } \\ & \square \text { Yes } & \square \text { No } \\ & \square \text { Yes } & \square \text { No }\end{array}$

Procedures of the Treatment 


\section{Alendronate}

$\begin{array}{lccc}\text { Major AEs } & \text { Frequency (\%) } & \text { Treatment Needed } & \text { Procedures of the Treatment } \\ \text { Upper GI effects } & \square Y \text { Yes } \square \text { No } & & - \\ \text { Esophagitis/esophagael ulcer } & \square Y \text { Yes } \square \text { No } & - \\ \text { Other } & \square Y \text { Y } & \square \text { No } & -\end{array}$

$>$ Raloxifene

Major AEs Frequency (\%) Treatment Needed Procedures of the Treatment

Leg cramps/DVT $\square \quad \square$ Yes $\square$ No

Other_— $\square$ - $\square$ Yes $\square$ No

Question II. What are the major considerations for choosing one therapy of the three?

Question III. Are there certain tests (e.g., biopsy, blood work, BMD, etc.) needed for follow-up or to maintain HRT, Raloxifene, or Alendronate therapies? Please specify name and average frequency of the tests. 
Question IV. How do the oral contraceptives affect women's risk of osteoporosis and fractures? Does the protective effect diminish rapidly after women stop taking them?

Question V. How do the hysterectomy and ovariectomy (before or after menopause) influence the risk of osteoporosis/osteoporotic fractures and preventive treatment decisions?

Comments/Suggestions about the Fracture Risk Survey: 


\section{Appendix D.}

\section{List of ICD-9 Codes Used in the Study}




\section{ICD-9 Codes}

Fracture, femur, neck (hip fracture)

$$
\begin{aligned}
& 733.14-733.15 \\
& 820.00-820.03 \\
& 820.09 \\
& 820.20-820.22 \\
& 820.8 \\
& 821.00-821.01 \\
& 821.20-821.23 \\
& 821.29
\end{aligned}
$$

Fracture, vertebral

733.13 (due to osteoporosis)

$805.00-805.08$

805.2

805.4

Fracture, wrist

814.00

733.12

Source: International Classification of Diseases $9^{\text {th }}$ Edition. 1999-2000. 


\section{Appendix E.}

\section{Cover Letters and Questionnaires}




\section{First Survey}

\section{Dear Madam,}

Osteoporosis is a disorder in which the bones become increasingly porous, brittle, and are likely to fracture. It is especially prevalent in postmenopausal women. In West Virginia (WV), there were a total of 183,962 women who suffered from either low bone mass or osteoporosis in 1996. Over one half of all women aged 50 and older in WV are at risk for osteoporosis or osteoporotic fractures (WV Bureau for Public Health, 1998).

To address this problem, researchers at West Virginia University School of Pharmacy are conducting a study to find out what factors are related to osteoporosis in WV Medicaid women. This study is part of a doctoral research project. Information obtained from this study could be useful in helping the Medicaid program understand the needs of those with osteoporosis and develop appropriate strategies to help them better.

Please find the attached questionnaire that asks you about your health and lifestyle factors related to the risk of osteoporotic fractures. Your participation in this study is voluntary and your response will not influence your Medicaid services. Although we hope that you could answer all of the questions, you do not have to answer any question that makes you uncomfortable. Your responses will be coded and your name will not appear in any data analysis or study reports. Therefore, we assure you of as much confidentiality as legally possible.

Your response will provide valuable information and is critical to the results of the study. We would appreciate it if you could kindly take a few minutes to complete the survey and return it in the postage-paid envelope. If you have any questions, please do not hesitate to contact us at (304) 293-6991 or 293-1652.

\section{Thank You.}

Sincerely,

Xin Gao, M.S.

Outcomes Research Fellow

Department of Pharmaceutical Systems and Policy
Suresh Madhavan, M.B.A, Ph.D. Associate Professor and Chairperson of Department of Pharmaceutical Systems and Policy 


\section{Second Survey}

\section{Dear Madam,}

About 2 weeks ago, we sent you a survey asking about your health and lifestyle factors related to the risk of osteoporosis and fractures. We have not yet received your completed survey.

We understand that you are busy or may not have received the survey. However, your views are extremely important to us, and the information obtained from this study would be very useful in helping the West Virginia Medicaid program understand the needs of women with osteoporosis, or who are at high risk of osteoporosis, to better help them. Therefore, we are again sending you this survey and would appreciate it very much if you could kindly take a few minutes to complete and return it in the postage-paid envelope.

Your participation in this study is voluntary and your response will not influence your Medicaid services in any way. Although we hope that you will answer all of the questions, you do not have to answer any question that makes you uncomfortable. Your responses will be coded, and your name will not appear in any data analysis or study reports. Therefore, we assure you of as much confidentiality as legally possible.

If you have any questions or concerns, please do not hesitate to contact Xin Gao at (304) 293-6991 or Dr. Suresh Madhavan at (304) 293-1652.

Thank You.

Sincerely,

Elizabeth S. Lawton

Commissioner 


\section{Questionnaire for Controls}

Instruction: This questionnaire asks you about your health, as well as risk factors related to bone weakness and fractures. Please answer each question by checking the box that best represents your situation. If you are not sure how to answer, please give the best answer that you can.

\section{SECTION A. HEALTH PROFILE}

1. In general, would you say your health is

$\square$ Excellent $\square$ Very Good $\square$ Good $\square$ Fair $\square$ Poor $\square$ Don't Know

2. Have you ever had an osteoporotic fracture? (Osteoporotic fracture is a broken bone due to weakened bones. It usually occurs after 45 years of age. The most common broken bones are back bone, wrist, and hip.) a Yes a No

If "Yes", at what age did you experience your first osteoporotic fracture?

If "Yes", did a doctor tell you that the fracture was related to osteoporosis? $\square$ Yes $\square$ No

3. Have you ever been told by a doctor that you have osteoporosis or osteopenia?

$\square$ Yes $\square$ No

If "Yes", approximately how long has it been since the diagnosis? year(s)

4. Do you suffer from any of the following illnesses?

Arthritis

$\square$ Diabetes

$\square$ Heart Disease $\square$ Asthma

$\square$ High Blood Pressure $\square$ Others, please specify

5. a) About how much do you weigh now without shoes? pounds

b) About how much did you weigh without shoes at age of 20-30 years? pounds

6. a) What is your height now without shoes? feet inches

b) What was your height without shoes at age of 20-30 years? feet __ inches

7. Bone mineral density (BMD) screening is a test for measuring bone mass in the spine, wrist, hip, or in the heel or hand. Have you ever had a BMD test?

$\square$ Yes $\square$ No 
8. Have you ever taken any of the following medications for menopausal symptoms such as hot flashes, or for bone weakness? (Please check all that apply)

$\square$ estrogens (such as Premarin ${ }^{\circledR}$ or Estrace $^{\circledR}$ ) $\quad \square$ Evista $^{\circledR} \quad \square$ Fosamax $^{\circledR}$

$\square$ calcitonin (such as Cibacalcin ${ }^{\circledR}$ or Miacalcin $^{\circledR}$ ) $\square$ other:

$\square$ I have NOT received any treatment for menopausal symptoms or bone weakness

9. Since you were 45 years of age, have you ever taken the following medications for more than 3 months? (Please check all that apply)

$\square$ Steroid pills such as Celestone ${ }^{\circledR}$, Cortisone, Deltasone ${ }^{\circledR}$, Prednisone, or Prelone ${ }^{\circledR}$

Thyroid medications such as Levothroid ${ }^{\circledR}$, Levoxyl, or Synthroid ${ }^{\circledR}$

Diuretics (water pills) such as Aquatensen ${ }^{\circledR}$, HydroDiuril $^{\circledR}$, HCTZ, or Oretic $^{\circledR}$

Sedatives such as Doriden $^{\circledR}$, Miltown $^{\circledR}$, Nembutal $^{\circledR}$, Seconal $^{\circledR}$, or Valium ${ }^{\circledR}$.

Antiseizure drugs such as Dilantin ${ }^{\circledR}$, Klonopin $^{\circledR}$, Phenobarb, Mysoline $^{\circledR}$, Tegretol $^{\circledR}$, or Zarontin ${ }^{\circledR}$

$\square$ I have NOT taken any of the above medications

\section{SECTION B. FAMILY AND REPRODUCTIVE HISTORY}

10. Do you have any female first-degree relatives (such as grandmother, mother or sisters) who have been diagnosed with osteoporosis, or have experienced height loss or fractures after 45 years of age?

$\square$ Yes $\square$ No

11. Have you ever taken oral contraceptives (birth control pills) for more than 1 year?

$\square$ Yes $\square$ No

If "Yes", how long have you been taking them or did you take them: __ year(s)

and if you are not using them now, how long ago did you stop taking them: __ year(s)

12. Have you reached menopause?

$\square$ Yes $\square$ No

If "Yes", What was your age at menopause? years

13. Have you had an operation to remove the uterus (womb)?

$\square$ Yes $\square$ No

If "Yes", at what age? years

14. Have you had an operation to remove an ovary/ovaries before natural menopause?

$\square$ Yes $\square$ No

If "Yes", at what age? years 


\section{SECTION C. GENERAL LIFESTYLE DATA}

15. On average, about how many servings of the following dairy products do you have per day? (Each serving is approximately equal to an 8 oz cup or 240 gms in weight)

\section{Number of Servings per day}

a. lowfat milk, skim milk, butter milk or dry milk /day

b. whole milk or evaporated milk /day

c. any kind of yogurt /day

d. cottage cheese

e. hard or soft cheese including cheese dishes such as macaroni and cheese /day

f. ice cream

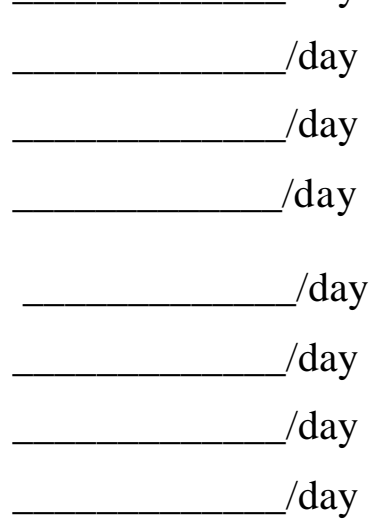

16. Do you take any calcium supplement (e.g., Caltrate, Os-Cal 500, or Tums) regularly*? $\square$ Yes $\square$ No If "Yes", for how long have you been taking these products regularly? year(s)

17. Do you take Vitamin D or cod liver oil or other fish oils regularly ${ }^{*}$ ?

$\square$ Yes $\square$ No If "Yes", for how long have you been taking them regularly? year(s)

18. Do you take any multivitamin pills including therapeutic and geriatric multivitamins regularly*?

$\square$ Yes $\square$ No

If "Yes", for how long have you been taking them regularly? year(s)

19. Do you exercise regularly* (such as running, walking, weight-bearing exercise, etc.)? $\square$ Yes $\square$ No If "Yes", for how long have you exercised regularly? year(s)

20. Does/Did your job involve intensive physical activities (such as lifting, lots of walking, or carrying heavy objects)?

$\square$ Yes $\square$ No

* "Regularly" means daily, or at least 3 times a week.

\section{(PLEASE GO TO THE NEXT PAGE)}


21. Do you smoke cigarettes?

$\square$ Never

$\square$ I did, but I quit $\square$ I smoke occasionally

I smoke every day (less than a pack)

$\square$ I smoke every day (more than a pack)

22. Do you drink alcoholic beverage such as beer, wine/wine coolers, or liquor?

$\square$ Never

I did, but I quit $\square$ I drink occasionally

I drink about 2-3 times a week

I drink daily/almost daily

\section{SECTION D. DEMOGRAPHICS AND RISK PERCEPTION}

23. How old were you at your last birthday? years.

24. What is the highest level of education that you have completed?

8th or less than 8th grade

9th to 12th grade or GED (high school graduate)

College 1 year to 3 years (some college or technical school)

$\square$ College 4 years or more (college graduate)

25. How would you rate your chance of having a fracture due to brittle bones in your future life time? (Please mark on the scale, where $0 \%$ means "I will definitely not develop a fracture", and 100\% means "I will definitely have a fracture".)

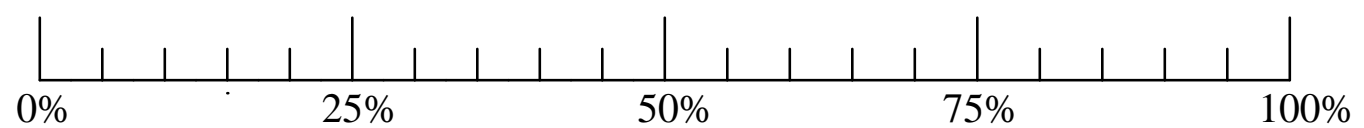

If you have any comments about osteoporosis/fractures, please write them below.

Please return the survey in the self-addressed envelope. No postage is required.

THANK YOU VERY MUCH FOR YOUR TIME AND EFFORT! WE WISH YOU A LONG, HAPPY, AND HEALTHY LIFE! 


\section{Questionnaire for Cases}

Instruction: This questionnaire asks you about your health, as well as risk factors related to bone weakness and fractures. Please answer each question by checking the box that best represents your situation. If you are not sure how to answer, please give the best answer that you can.

\section{SECTION A. HEALTH PROFILE}

1. In general, would you say your health is

$\square$ Excellent $\square$ Very Good $\square$ Good $\square$ Fair $\square$ Poor $\square$ Don't Know

2. Have you ever had an osteoporotic fracture? (Osteoporotic fracture is a broken bone due to weakened bones. It usually occurs after 45 years of age. The most common broken bones are back bone, wrist, and hip.)

\section{$\square$ Yes $\square$ No}

If "Yes", at what age did you experience your first osteoporotic fracture?

If "Yes", did a doctor tell you that the fracture was related to osteoporosis? $\overline{\square \text { Yes }} \square$ No

3. Have you ever been told by a doctor that you have osteoporosis or osteopenia?

$\square$ Yes $\square$ No

If "Yes", approximately how long has it been since the diagnosis? year(s)

4. Do you suffer from any of the following illnesses?
$\square$ Arthritis
$\square$ Asthma
$\square$ Diabetes
$\square$ High Blood Pressure
$\square$ Heart Disease
$\square$ Others, please specify

5. a) About how much do you weigh now without shoes? pounds

b) About how much did you weigh without shoes at age of 20-30 years? pounds

6. a) What is your height now without shoes? feet inches

b) What was your height without shoes at age of 20-30 years? feet ___inches

7. Bone mineral density (BMD) screening is a test for measuring bone mass in the spine, wrist, hip, or in the heel or hand. Did you have a BMD test before your first osteoporotic fracture?

$\square$ Yes $\square$ No 
8. Did you take any of the following medications for menopausal symptoms such as hot flashes, or for bone weakness before your first osteoporotic fracture? (Please check all that apply)

\begin{tabular}{|c|c|c|}
\hline$\square$ estrogens (such as Premarin ${ }^{\circledR}$ or Estrace $^{\circledR}$ ) & $\square$ Evista $^{\circledR}$ & $\square$ Fosamax $^{\circledR}$ \\
\hline nin (such as Cibacalcin ${ }^{\circledR}$ or Miacalcin ${ }^{\circledR}$ ) & $\square$ other: & \\
\hline
\end{tabular}

I did NOT received any treatment for menopausal symptoms or bone weakness before my first osteoporotic fracture

9. Had you ever taken the following medications for more than 3 months before your first osteoporotic fracture (but after 45 years of age)? (Please check all that apply)

$\square$ Steroid pills such as Celestone ${ }^{\circledR}$, Cortisone, Deltasone ${ }^{\circledR}$, Prednisone, or Prelone ${ }^{\circledR}$

Thyroid medications such as Levothroid ${ }^{\circledR}$, Levoxyl, or Synthroid ${ }^{\circledR}$

$\square$ Diuretics (water pills) such as Aquatensen $^{\circledR}$, HydroDiuril $^{\circledR}$, HCTZ, or Oretic $^{\circledR}$

$\square$ Sedatives such as Doriden ${ }^{\circledR}$, Miltown $^{\circledR}$, Nembutal $^{\circledR}$, Seconal $^{\circledR}$, or Valium ${ }^{\circledR}$.

$\square$ Antiseizure drugs such as Dilantin ${ }^{\circledR}$, Klonopin $^{\circledR}$, Phenobarb, Mysoline $^{\circledR}$, Tegretol $^{\circledR}$, or Zarontin ${ }^{\circledR}$

$\square$ I have NOT taken any of the above medications

\section{SECTION B. FAMILY AND REPRODUCTIVE HISTORY}

10. Do you have any female first-degree relatives (such as grandmother, mother or sisters) who have been diagnosed with osteoporosis, or have experienced height loss or fractures after 45 years of age?
$\square$ Yes
$\square$ No

11. Have you ever taken oral contraceptives (birth control pills) for more than 1 year?

$\square$ Yes $\square$ No

If "Yes", how long have you been taking them or did you take them: year(s)

and if you are not using them now, how long ago did you stop taking them: year(s)

12. Have you reached menopause?

$\square$ Yes $\square$ No If "Yes", What was your age at menopause? years

13. Have you had an operation to remove the uterus (womb)?

$\square$ Yes $\square$ No If "Yes", at what age? years

14. Have you had an operation to remove an ovary/ovaries before natural menopause?

$\square$ Yes $\square$ No If "Yes", at what age? years 


\section{SECTION C. GENERAL LIFESTYLE DATA}

15. On average, about how many servings of the following dairy products did you have per day before your first osteoporotic fracture?

(Each serving is approximately equal to an $8 \mathrm{oz}$ cup or $240 \mathrm{gms}$ in weight)

\section{Number of Servings per day}
a. lowfat milk, skim milk, butter milk or dry milk
b. whole milk or evaporated milk
c. any kind of yogurt
d. cottage cheese
e. hard or soft cheese including cheese dishes such as macaroni and cheese
f. ice cream
g. cream, half and half, or sour cream
h. orange juice containing calcium /day /day /day /day /day /day /day /day

16. Did you take any calcium supplement (e.g., Caltrate, Os-Cal 500, or Tums) regularly* before your first osteoporotic fracture?

$\square$ Yes $\square$ No

If "Yes", for how long had you been taking these products regularly? year(s)

17. Did you take Vitamin D or cod liver oil or other fish oils regularly ${ }^{*}$ before your first osteoporotic fracture?

$\square$ Yes $\square$ No

If "Yes", for how long had you been taking them regularly? year(s)

18. Did you take any multivitamin pills including therapeutic and geriatric multivitamins regularly ${ }^{*}$ before your first osteoporotic fracture?

$\square$ Yes $\square$ No

If "Yes", for how long had you been taking them regularly? year(s)

19. Did you exercise (such as running, walking, weight-bearing exercise, etc) regularly ${ }^{*}$ before your first osteoporotic fracture?

$\square$ Yes $\square$ No

If "Yes", for how long had you exercised regularly? year(s)

* "Regularly" means daily, or at least 3 times a week.

(PLEASE GO TO THE NEXT PAGE) 
20. Did your job involve intensive physical activities (such as lifting, lots of walking, or carrying heavy objects) before your first osteoporotic fracture ?

$\square$ Yes $\square$ No

21. Did you smoke cigarettes before your first osteoporotic fracture?

Never smoked

$\square$ I did, but I quit $\square$ I smoked occasionally

I smoked every day (less than a pack)

$\square$ I smoked every day (more than a pack)

22. Did you drink alcoholic beverage such as beer, wine/wine coolers, or liquor before your first osteoporotic fracture?

$\square$ Never drank alcohol

$\square$ I did, but I quit

I drank occasionally
I drank about 2-3 times a week
I drank daily/almost daily

\section{SECTION D. DEMOGRAPHICS}

23. How old were you at your last birthday? years.

24. What is the highest level of education that you have completed?

$\square$ 8th or less than 8th grade

$\square$ 9th to 12th grade or GED (high school graduate)

$\checkmark$ College 1 year to 3 years (some college or technical school)

$\square$ College 4 years or more (college graduate)

25. How would you rate your chance of having a fracture due to brittle bones in your future life time? (Please mark on the scale, where $0 \%$ means "I will definitely not develop another fracture", and 100\% means "I will definitely have another fracture".)

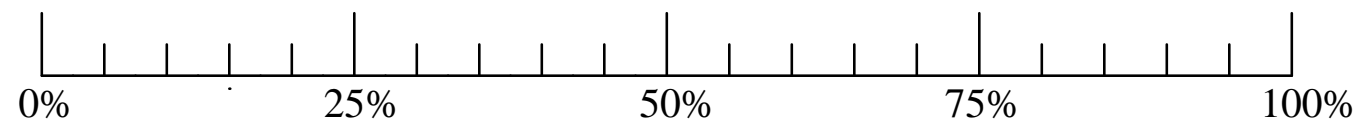

If you have any comment about osteoporosis/fractures, please write them below.

Please return the survey in the self-addressed envelope. No postage is required.

THANK YOU VERY MUCH FOR YOUR TIME AND EFFORT! WE WISH YOU A LONG, HAPPY, AND HEALTHY LIFE! 


\section{Appendix F.}

Non-Respondent Questionnaire 
Dear Madam,

About 2 weeks ago, we sent you a survey about your health and lifestyle factors related to the risk of osteoporosis and fractures. We have not yet received your completed survey. Since your views are extremely important to us, we would like to know your reason for not responding and some key information for the study. Please answer the few questions below and mail it to us in the business reply envelope provided. If you have any questions please call us at (304) 293-6991 or 293-1652.

Sincerely,

Cindy (Xin) Gao, M.S. Outcomes Research Fellow
Suresh Madhavan, M.B.A, Ph.D.

Associate Professor and Chairperson

$* * * * * * * * * * * * * * * * * * * * * * * * * * * * * * * * * * * * * * * * * * * * * * * * * * * * * * * * * * * * * * * * * * * * * * * * * * *$

1. I did not respond to the survey because:

$\square$ I did not receive it

$\square$ I was not at home

$\square$ I did not have time to complete it

$\square$ Lost the survey

$\square$ I haven't finished it yet
I do not respond to mail surveys

$\square$ The survey was too long

The survey was confusing

$\square$ I am not interested in this issue

$\square$ Other reasons (Please specify):

2. In general, your health is: $\square$ Excellent $\square$ Very Good $\square$ Good $\square$ Fair $\square$ Poor

3. Have you ever been told by your physician that you have osteoporosis? $\square$ Yes $\square$ No

4. Have you ever had a fracture since you were 45 years old? $\square$ Yes $\square$ No

5. Have you reached menopause? $\square$ Yes $\square$ No If "Yes", age at menopause years

6. Your current age: years

7. About how much do you weigh?

pounds (or kgms)

8. What is your height? feet inches (or $\mathrm{cm})$

9. How would you rate your chance of having a fracture due to brittle bones in your life time? (Please mark on the ruler, 0\% means "I will definitely not develop a fracture, $100 \%$ means "I will definitely have a fracture".)

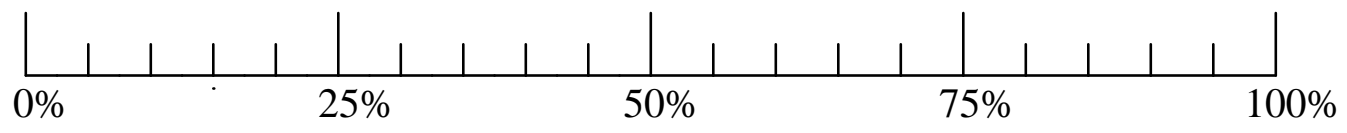




\section{Appendix G.}

NDC Codes Used in the Study 


\section{Alendronate sodium}

Fosamax

00006-0925-31

00006-0925-58

00006-0936-31

00006-0936-28

00006-0936-58

00006-0936-72

00006-0936-82

00006-0212-31

54868-3857-00

Calcitonin, salmon

00070-4492-01

00186-1608-13

Calcimar

00075-1306-01

54569-1994-00

Miacalcin

00078-0149-23

00078-0311-90

Etidronate disodium

Didronel

00149-0405-60

00149-0406-60

54569-2695-00

54868-2981-00

58063-0457-01

\section{Raloxifene hydrochloride}

Evista

00002-4165-30

00002-4165-02 


\section{Curriculum Vitae}

\section{Xin Gao}

PPD DEVELOPMENT, MORRISVILLE, NC

Pharmacoeconomist

November 2000 - Present

Study design, project development/completion, data analysis, and result summary/report (or assists in these tasks) in the research related to pharmacoeconomics, quality of life, and other outcomes and/or marketing studies.

\section{PFIZER INC., NY}

$\underline{\text { Summer Intern }}$

May 2000 - August 2000

- A wellness evaluation project to measure the indirect costs due to lost productivity for 11 common health conditions.

- A large retrospective study aimed to examine the relationship of various risk factors and erectile dysfunction (ED) status, and to establish a risk assessment model for ED.

- A Community Acquired Pneumonia (CAP) project to examine the effects of different regimens on hospital length-of-stay (LOS).

- Business booklet for the Coronary Heart Disease Risk Assessment Model.

\section{WEST VIRGINIA UNIVERSITY, DEPARTMENT OF PHARMACEUTICAL SYSTEMS \& POLICY, SCHOOL OF PHARMACY, MORGANTOWN, WV}

Pfizer Health Outcomes Research Fellow

August 1999 - October 2000

- Dissertation project: Economic evaluation of three preventive drug therapies for osteoporotic fractures among women with different risk levels. A cost-effectiveness analysis is conducted using a decision model and Monte Carlo simulations.

- Dissertation study on a risk assessment model for osteoporotic fracture among WV Medicaid postmenopausal women.

- Using the Health Plan claims data to examine the effect of co-payments on medical care utilization and expenditures among diabetic patients.

- Using the Health Plan claims data to examine the relationship between health care expenditures and compliance, endocrinologist visit, and care received among type-II diabetic patients. 
- Lecturing on topics such as decision analysis (using Treeage), risk assessment, and sensitivity analysis in Pharmacoeconomics class in spring semester, 2000.

\section{WEST VIRGINIA UNIVERSITY, PERVENTION RESEARCH CENTER, MORGANTOWN, WV}

Research Assistant

August 1997 - July 1999

$\underline{\text { Summer Intern }}$

May 1997 - July 1997

- Developing and evaluating smoking cessation interventions.

- Developing instruments for various study designs.

- Literature review, data analysis, and manuscript writing.

- Thesis study examining the relationship between disease severity, health beliefs, and treatment adherence among HIV patients.

- Using time series analysis to evaluate trends in Medicare and Medicaid payments on nursing home care and home health care: $1975-1995$.

- A cost-benefit analysis and quality-of-life assessment for the use of filgrastim (GCSF) in HIV/AIDS patients with antiretrovirals-induced neutropenia.

\section{WEST VIRGINIA UNIVERSITY, DEPARTMENT OF PHARMACEUTICAL SYSTEMS \& POLICY, SCHOOL OF PHARMACY, MORGANTOWN, WV}

Teaching Assistant

August 1996 - April 1997

- Assisting faculties in teaching pharmaceutical care and disease management, and pharmacy law.

\section{CHINESE ACADEMY OF MEDICAL SCIENCES, CARDIOVASCULAR INSTITUTE AND FUWAI HOSPITAL, DEPARTMENT OF PHARMACY, BEIJING, CHINA}

Pharmacist

August 1994 - July 1996

- Completing the rotation in out-patient pharmacy, in-patient pharmacy, emergency pharmacy, and clinical pharmaceutics and pharmcokinetic laboratory.

- Distributing medications and providing basic pharmaceutical care. 


\section{EDUCATION}

- 2001

- 1999

- 1994
Ph.D. in Department of Pharmaceutical Systems and Policy, School of Pharmacy, Morgantown, West Virginia University.

M.S. in Department of Pharmaceutical Systems and Policy, Morgantown, West Virginia University.

B.S. in Pharmaceutical Sciences, China Pharmaceutical University, Nanjing, China.

\section{PROFESSIONAL DEVELOPMENT}

Training received while employed at PPD is available upon request.

Prior to PPD:

Managed Care Training (8/2000) given by Pfizer, Inc.

Health Outcomes Research 1-day seminar (7/2000) given by the Outcome Research Group (Marketing Department) of Pfizer, Inc.

Risk Management workshop (7/2000) provided by Pfizer, Inc.

Applied Pharmacoeconomics workshop (5/2000) workshop conducted by the International Society for Pharmacoeconomics.

Osteoporosis Management workshop (1/2000) conducted by school of nursing, West Virginia University, Morgantown, WV.

Reference Manager workshop (12/1999) conducted by the Computer Based Learning Center (CBLC), West Virginia University, Morgantown, WV.

Access and HTML summer workshops (6/99-7/99) offered by the Computer Based Learning Center (CBLC), West Virginia University, Morgantown, WV.

SAS summer workshops (6/98-7/98) on offered by the Computer Based Learning Center (CBLC), West Virginia University, Morgantown, WV.

\section{PROFESSIONAL AFFILIATIONS}

International Society for Pharmacoeconimics and Outcomes Research, January 1999 - Present American Pharmaceutical Association, January 1999 - Present 


\section{HONORS}

- $\quad$ Graduate Research Award 1999

- $\quad$ Graduate Student Representative of School of Pharmacy

- Vice-President of WVU-ISPOR Student Chapter

- $\quad$ Member of Rho Chi Honor Society

\section{COMPUTER SKILLS}

SPSS, JUMP, SAS, LISREL; Analysis Package for Economics: EVIEWS, GAMS; Decision Analysis: Tree-Age, @Risk; Other: SurveyPro, Excel, Access, Word, Power Point, Word Perfect.

\section{PUBLICATIONS}

Horn K, Gao X, Williams J. Conjoint alcohol and tobacco use: A case for dual substance intervention. Academic Emergency Medicine. 2001; (in press).

Gao X, Nau D. The congruence of three self-report measures of medication adherence among HIV patients. The Annals of Pharmacotherapy. 2000;34(10):1117-22.

Gao X, Nau D, Rosenbluth S. The relationship of disease severity, health beliefs, and medication adherence among HIV patients. AIDS Care. 2000;12(4):387-98.

Horn K, Gao X, Dino G. Determinants of youth tobacco use in West Virginia: A comparison of smoking and smokeless tobacco use. American Journal of Drug and Alcohol Abuse.

2000;26(1):125-38.

Horn K, Maniar S, Dino G, Gao X, Sayers K. Coaches attitudes toward smokeless tobacco \& intentions to intervene with athletes. Journal of School Health. 2000;70(3):89-94.

Horn K, Dino G, Gao X, Momani A. Feasibility evaluation of Not-On-Tobacco: The American Lung Association's new stop smoking cessation program for adolescents. Health Education. 1999; $\operatorname{Sep}(5): 192-206$.

Gao X, Chen J. Strategies for transnational management in pharmaceutical enterprises. Journal of Pharmaceutical Economics. 1995,2:27-30.

Chen J, Gao X. Suggestion to national policies about improving the transnational management in pharmaceutical enterprises. Journal of Pharmacy in China. 1994;1:15-6. 


\section{PRESENTATIONS}

Gao X, Madhavan S, Nau D, Ambegaonkar A, Islam S, Rosenbluth S, Amonkar M. Applying background risk distribution to evaluate the Cost-effectiveness of Three Preventive Drug Therapies for Osteoporotic Fractures. Poster presentation at the 6th International Society of Pharmacoeconomics and Outcomes Research (ISPOR) Annual Meeting. Arlington, Virginia. May 2001.

Gao X, Madhavan S, Nau D, Ambegaonkar A, Islam S, Rosenbluth S, Amonkar M. A Risk Assessment Model for Osteoporotic Fracture in Postmenopausal Women. Oral presentation at the Drug Information Association "Pharmaceutical Outcomes Research" Meeting. Savannah, GA. April 2001.

Gao X, Madhavan S. Cost-effectiveness analysis of three preventive drug therapies for osteoporotic fractures among postmenopausal women. Poster presentation at the 148th American Pharmaceutical Association (APhA) Annual Meeting. San Francisco, CA. March 2001.

Gao X, Nau D. A Comparison of three self-report measures of medication adherence among HIV patients. Poster presentation at the 147th APhA Annual Meeting. Washington D.C., March 2000 .

Dino G, Horn K, Gao X, Goldcamp J. The feasibility and efficacy of Not-On-Tobacco: A teen smoking cessation trial in FL. Poster presented at the 12th National Conference on Nicotine Dependence. The American Society of Addiction Medicine. Cleveland, Ohio, October 1999.

Horn K, Williams J, Hungerford D, Gao X, Helmkamp J, Furbee M, Manley B. Conjoint alcohol and tobacco use: An opportunity for dual intervention? The 12th National Conference on Nicotine Dependence. The American Society of Addiction Medicine. Cleveland, Ohio, October 1999.

Horn K, Maniar S, Dino G, Gao X, Sayers K. Smokeless tobacco \& sport in WV: Coaches' attitudes \& intentions to intervene. Poster presented at the 12th National Conference on Nicotine Dependence. The American Society of Addiction Medicine. Cleveland, Ohio, October 1999.

Horn K, Dino G, Momani A, Gao X. Not-On-Tobacco: Smoking Cessation Program. Poster at the American Association for Health Plans 2nd Annual Meeting. San Diego, CA, January 1999.

Gao X, Nau D, Rosenbluth S. The relationship of disease severity, health beliefs, and medication adherence among HIV patients. The 145th APhA Annual Meeting. Miami, FL, March 1998. 